\title{
THE BOLOCAM GALACTIC PLANE SURVEY. VII. CHARACTERIZING THE PROPERTIES OF MASSIVE STAR-FORMING REGIONS
}

\author{
Miranda K. Dunham ${ }^{1,2}$, Erik Rosolowsky ${ }^{3}$, Neal J. Evans II $^{2}$, Claudia Cyganowski ${ }^{4,6}$, and James S. Urquhart ${ }^{5}$ \\ ${ }^{1}$ Department of Astronomy, Yale University, New Haven, CT 06520-8101, USA; miranda.dunham @yale.edu \\ 2 Department of Astronomy, The University of Texas at Austin, Austin, Texas 78712-0259, USA \\ ${ }^{3}$ Department of Physics and Astronomy, University of British Columbia, Okanagan, Kelowna BC V1V 1V7, Canada \\ ${ }^{4}$ Harvard-Smithsonian Center for Astrophysics, Cambridge, MA 02138, USA \\ ${ }^{5}$ CSIRO Astronomy and Space Science, Epping, NSW 1710, Australia \\ Received 2011 May 10; accepted 2011 August 5; published 2011 October 24
}

\begin{abstract}
We present the results of a Green Bank Telescope survey of $\mathrm{NH}_{3}(1,1),(2,2),(3,3)$ lines toward 631 Bolocam Galactic Plane Survey (BGPS) sources at a range of Galactic longitudes in the inner Galaxy. We have detected the $\mathrm{NH}_{3}(1,1)$ line toward $72 \%$ of our targets (456), demonstrating that the high column density features identified in the BGPS and other continuum surveys accurately predict the presence of dense gas. We have determined kinematic distances and resolved the distance ambiguity for all BGPS sources detected in $\mathrm{NH}_{3}$. The BGPS sources trace the locations of the Scutum and Sagittarius spiral arms, with the number of sources peaking between $R_{\mathrm{Gal}} \sim 4$ and $5 \mathrm{kpc}$. We measure the physical properties of each source and find that depending on the distance, BGPS sources are primarily clumps, with some cores and clouds. We have examined the physical properties as a function of Galactocentric distance, and find a mean gas kinetic temperature of $15.6 \mathrm{~K}$, and that the $\mathrm{NH}_{3}$ column density and abundance decrease by nearly an order of magnitude between $R_{\text {Gal }} \sim 3$ and $11 \mathrm{kpc}$. Comparing sources at similar distances demonstrates that the physical properties are indistinguishable, which suggests a similarity in clump structure across the Galactic disk. We have also compared the BGPS sources to criteria for efficient star formation presented independently by Heiderman et al. and Lada et al., and for massive star formation presented by Kauffmann et al. Forty-eight percent of our sample should be forming stars (including massive stars) with high efficiency, and $87 \%$ contain subregions that should be efficiently forming stars. Indeed, we find that $67 \%$ of the sample exhibit signs of star formation activity based on an association with a mid-infrared source.
\end{abstract}

Key words: dust, extinction - ISM: clouds - ISM: molecules - stars: formation

Online-only material: color figures, figure set, machine-readable tables

\section{INTRODUCTION}

Massive stars $\left(M>8 M_{\odot}\right)$ play a significant role in the evolution of their immediate environment, as well as in evolution on a galaxy-wide scale via their many feedback processes including strong $\mathrm{UV}$ radiation that creates $\mathrm{H}$ II regions, outflows and winds which inject energy back into the surrounding medium, and supernova at the end of their lives (Kennicutt 2005). Given the importance of massive stars in galactic evolution, it is imperative that we understand how and where massive stars form within the Milky Way in order to interpret observations of other galaxies.

Large sample sizes are beneficial in this task, and many large samples have been chosen in various ways. For example, Plume et al. (1992) selected $179 \mathrm{H}_{2} \mathrm{O}$ masers from Cesaroni et al. (1988) and followed up with observations of dense gas tracers (CS $J=7-6$ and $\mathrm{CO} J=3-2$ ). This $\mathrm{H}_{2} \mathrm{O}$ maser sample was followed up with lower $J$ transitions of CS (Plume et al. 1997; Shirley et al. 2003) as well as submillimeter dust continuum (Mueller et al. 2002). Shirley et al. (2003) thoroughly characterized the physical properties based on CS $J=5-4$ maps of a subsample of 64 sources from Plume et al. (1992). They found the following median properties: $R=0.32 \mathrm{pc}$, $M_{\text {vir }}=920 M_{\odot}$, and $\Sigma=0.60 \mathrm{~g} \mathrm{~cm}^{-2}$. Plume et al. (1997) found a mean volume density of $\left\langle\log \left(n / \mathrm{cm}^{-3}\right)\right\rangle=5.9$ based on multiple transitions of CS. Recently, Wu et al. (2010) have characterized properties for a subsample of 50 massive,

\footnotetext{
${ }^{6}$ NSF Astronomy and Astrophysics Postdoctoral Fellow.
}

dense clumps from Plume et al. (1992) based on HCN and CS line emission. They found a range for the mean of each property based on the different molecular line tracers used: $\left\langle M_{\mathrm{vir}}\right\rangle=1300-5300 M_{\odot},\langle\Sigma\rangle=0.29-1.09 \mathrm{~g} \mathrm{~cm}^{-2}$, and $\langle n\rangle=3.2 \times 10^{4}-2.5 \times 10^{5} \mathrm{~cm}^{-3}$. Other samples were defined based on the presence of ultracompact $\mathrm{H}_{\text {II }}$ (UC H II) regions and IRAS colors. Wood \& Churchwell (1989b) complied a catalog of massive star-forming regions from the IRAS All Sky Survey based on typical far-IR colors of the cool dust surrounding UC H II regions (Wood \& Churchwell 1989a). Beuther et al. (2002) compared $1.2 \mathrm{~mm}$ continuum observations and CS molecular line studies toward $69 \mathrm{UC}$ H II regions based on the sample defined by Sridharan et al. (2002). Beuther et al. determined physical properties similar to those of Shirley et al. (2003).

These initial studies of massive star formation were important first steps toward characterizing large samples, although the methods employed in identifying the sources resulted in highly biased samples. Requiring the presence of $\mathrm{H}_{2} \mathrm{O}$ masers or UC HII regions biases the samples to a particular stage in star formation. Both phenomena require a significant source of energy, and therefore will bias the samples to later evolutionary states when a protostar has already formed. Therefore, the properties determined by these initial studies may not be characteristic of massive star-forming regions at all evolutionary stages because of these biases.

The many large-scale, blind Galactic plane surveys recently completed and currently underway provide the data sets needed to study massive star formation in a Galactic context. In 
particular, three large-scale surveys have recently done so: the Boston-University Five College Radio Astronomy Observatory ${ }^{13}$ CO Galactic Ring Survey (GRS; Jackson et al. 2006), the RedMSX Source (RMS; Hoare et al. 2004; Urquhart et al. 2008) Survey, and the Bolocam Galactic Plane Survey (BGPS; Aguirre et al. 2011).

Rathborne et al. (2009) identified 829 clouds and 6124 clumps within the ${ }^{13} \mathrm{CO}$ emission in the GRS. They compared properties of clouds inside and outside of the $5 \mathrm{kpc}$ molecular ring, a Galactic structure identified as an overdensity in molecular gas located at a Galactocentric radius of $5 \mathrm{kpc}$ that contains the majority of star formation in the Milky Way (e.g., Burton et al. 1975; Scoville \& Solomon 1975; Cohen \& Thaddeus 1977; Robinson et al. 1984; Clemens et al. 1988; Kolpak et al. 2002). They found that clouds within the $5 \mathrm{kpc}$ molecular ring have warmer temperatures, higher column densities, larger areas, and contained more clumps than clouds located outside of the molecular ring. Roman-Duval et al. (2009) resolved the distance ambiguity to 750 of the GRS clouds and found that their positions are consistent with a four-arm model of the Galaxy and trace the positions of the Scutum-Crux and Perseus spiral arms. Roman-Duval et al. (2010) derived physical properties of 580 of the GRS clouds and demonstrated that the gas surface density peaks within the $5 \mathrm{kpc}$ molecular ring. They found cloud sizes ranging from 0.1 to $40 \mathrm{pc}$, masses ranging from 10 to $10^{6} M_{\odot}$, a mean volume density of $230 \pm 21 \mathrm{~cm}^{-3}$ with the highest density being $\sim 10^{3} \mathrm{~cm}^{-3}$, and a mean surface density of $144 \pm 3 M_{\odot} \mathrm{pc}^{-2}$.

The RMS team has identified a candidate list of $\sim 2000$ massive young stellar objects (MYSOs) and UC H II regions based on MSX and IRAS colors (Lumsden et al. 2002). Urquhart et al. (2011) have recently compared the radial velocities of the MYSOs to the clouds identified in the GRS and adopted the kinematic distances determined by Roman-Duval et al. (2009), thereby obtaining a complete sample of 196 RMS sources with luminosities above their completeness limit of $\sim 10^{4} L_{\odot}$. The clouds containing an RMS source were found to be larger, more massive, and more turbulent than the GRS clouds without an RMS source. The subsample of GRS clouds containing RMS sources are also well correlated with the Galactic spiral arm structure.

The BGPS (Aguirre et al. 2011) has detected $1.1 \mathrm{~mm}$ thermal dust emission from the dense regions closely associated with star formation. Dunham et al. (2010; hereafter referred to as D10) found mean $\mathrm{H}_{2}$ column densities of $1.2 \times 10^{22} \mathrm{~cm}^{-2}$ for BGPS sources located within the Gemini OB1 molecular cloud, and Schlingman et al. (2011) found a mean $\mathrm{H}_{2}$ column density of $6.8 \times 10^{21} \mathrm{~cm}^{-2}$ for BGPS sources detected in $\mathrm{HCO}^{+}(3-2)$. In contrast to previous large-scale studies of massive star-forming regions, the BGPS is identifying high column density regions regardless of star formation signposts (such as $\mathrm{H}_{2} \mathrm{O}$ maser emission). By identifying sources based on dense gas and dust, the bias toward a particular evolutionary stage is avoided.

In this paper, we present the results of a targeted survey of $\mathrm{NH}_{3}$ toward 631 BGPS sources in the first Galactic quadrant. The goals of this paper are to determine the location of a large sample of BGPS sources within the Galaxy, characterize the physical properties of a large relatively unbiased sample of star-forming regions, study their physical properties as a function of environment using Galactocentric radius as a proxy, and to characterize their star formation potential and midIR source content. Section 2 briefly describes the BGPS and source selection, and Section 3 describes the $\mathrm{NH}_{3}$ setup and observations. We present basic results of the $\mathrm{NH}_{3}$ survey in Section 4. In Section 5, we determine kinematic distances and break the kinematic distance ambiguity (KDA). Section 6 includes a description of each physical quantity derived from the BGPS and $\mathrm{NH}_{3}$ surveys. Section 7 explores the trends in physical properties as a function of Galactocentric radius, and Section 8 includes a discussion of the star formation activity, comparison to other large-scale studies, and a discussion of whether BGPS sources are forming massive stars. Finally, Section 9 provides a summary of this work.

\section{THE BOLOCAM $1.1 \mathrm{~mm}$ GALACTIC PLANE SURVEY}

The BGPS ${ }^{7}$ has observed approximately $170 \mathrm{deg}^{2}$ of the northern Galactic plane in $1.1 \mathrm{~mm}$ continuum emission using Bolocam at the Caltech Submillimeter Observatory ${ }^{8}$ (CSO). The survey area consists of a continuous region spanning $-10^{\circ}<\ell<90^{\circ}$ and $|b| \leqslant 0.5$ as well as select regions of known star formation in the outer Galaxy: the Perseus tangent, the W3/4/5 region, and the Gemini OB 1 molecular cloud. The survey methods and data reduction are thoroughly described in Aguirre et al. (2011), and the source extraction algorithm and catalog are described in Rosolowsky et al. (2010). The BGPS has identified 8358 continuum emission sources over the entire coverage area (Rosolowsky et al. 2010), most of which were previously unknown. Here, we discuss details relevant to this work and refer the reader to these papers for further details.

The effective FWHM beam size of the BGPS is 33", slightly larger than the nominal Bolocam beam at $1.1 \mathrm{~mm}$ due to combining multiple observations of each field. The FWHM beam size corresponds to a solid angle of $2.9 \times 10^{-8} \mathrm{sr}$, which is equivalent to a top-hat function with a $40^{\prime \prime}$ diameter $\left(\Omega=2.95 \times 10^{-8} \mathrm{sr}\right)$. The BGPS catalog algorithm, Bolocat, presents aperture flux densities within 40", 80", and 120" diameter apertures representing the flux density within one $\left(S_{v}\left(40^{\prime \prime}\right)\right)$, two $\left(S_{v}\left(80^{\prime \prime}\right)\right)$, and three $\left(S_{v}\left(120^{\prime \prime}\right)\right)$ times the beam, respectively. Bolocat additionally provides an integrated flux density $\left(S_{v}\right.$ (int)), which is simply the sum of all pixels assigned to a given BGPS source and more accurately represents the flux densities of irregularly shaped sources. The aperture-based flux densities of point sources require correction factors (1.46 for $S_{v}\left(40^{\prime \prime}\right), 1.04$ for $S_{v}\left(80^{\prime \prime}\right)$, and 1.01 for $S_{v}\left(120^{\prime \prime}\right)$ ) to account for power falling outside of the aperture due to the sidelobes of the beam. $S_{v}$ (int) does not require a correction since it is not aperture based.

In addition to the aperture corrections, a flux calibration factor of $1.5 \pm 0.15$ is required. This factor is based on a comparison of BGPS data with $1.2 \mathrm{~mm}$ data (Rathborne et al. 2006; Motte et al. 2003, 2007; Matthews et al. 2009) acquired at different facilities, which showed that the BGPS flux densities were systematically lower (Aguirre et al. 2011). We apply the flux calibration factor in order to bring the BGPS flux densities into agreement with the $1.2 \mathrm{~mm}$ surveys. The aperture corrections and flux calibration factor are included in the flux densities presented here.

Bolocat includes coordinates of both a geometric centroid and the peak $1.1 \mathrm{~mm}$ emission. The major $\left(\sigma_{\text {maj }}\right)$ and minor $\left(\sigma_{\min }\right)$ axes of each source are calculated based on emission weighted moments, and the effective radius $(R)$ of each source is calculated as the geometric mean of the deconvolved major and minor axes:

$$
R=\eta\left[\left(\sigma_{\text {maj }}^{2}-\sigma_{\text {beam }}^{2}\right)\left(\sigma_{\text {min }}^{2}-\sigma_{\text {beam }}^{2}\right)\right]^{1 / 4},
$$

\footnotetext{
7 See http://irsa.ipac.caltech.edu/data/BOLOCAM_GPS/

8 The Caltech Submillimeter Observatory is supported by the NSF.
} 
Table 1

Observed $1.1 \mathrm{~mm}$ Properties

\begin{tabular}{|c|c|c|c|c|c|c|c|c|c|c|c|}
\hline $\begin{array}{l}\text { ID } \\
\text { Number }\end{array}$ & Source & $\begin{array}{l}\text { R.A. } \\
\text { (J2000) }\end{array}$ & $\begin{array}{c}\text { Decl. } \\
\text { (J2000) }\end{array}$ & $\begin{array}{l}\text { Peak R.A. } \\
\text { (J2000) }\end{array}$ & $\begin{array}{l}\text { Peak Decl. } \\
(\text { J2000) }\end{array}$ & $\begin{array}{c}R_{\text {major }} \\
\left(^{\prime \prime}\right)\end{array}$ & $\begin{array}{c}R_{\text {minor }} \\
\left({ }^{\prime \prime}\right)\end{array}$ & $\begin{array}{c}\text { P.A. } \\
\left({ }^{\circ}\right)\end{array}$ & $\begin{array}{c}R_{\text {obj }} \\
\left({ }^{\prime \prime}\right)\end{array}$ & $\begin{array}{c}S_{v}\left(120^{\prime \prime}\right) \\
(\mathrm{mJy})\end{array}$ & $\begin{array}{c}S_{v}(\text { int }) \\
(\mathrm{mJy})\end{array}$ \\
\hline 1307 & G007.501+00.001 & 180230.6 & -222800.3 & 180230.0 & -222807.0 & 23.4 & 18.2 & 173 & 35.41 & $399(110)$ & $356(94)$ \\
\hline 1309 & G007.509+00.403 & 180100.2 & -221603.4 & 180100.4 & -221546.3 & 27.1 & 15.3 & 154 & 28.73 & $550(140)$ & $588(110)$ \\
\hline 1310 & G007.564-00.042 & 180246.4 & -222612.5 & 180247.8 & -222605.6 & 28.2 & 21.6 & 34 & 48.07 & $629(170)$ & $533(170)$ \\
\hline 1311 & G007.600-00.142 & 180314.7 & -222707.6 & 180315.0 & -222710.1 & 25.5 & 15.0 & 142 & 25.78 & $717(160)$ & $681(120)$ \\
\hline 1313 & G007.622-00.000 & 180246.8 & -222146.5 & 180245.8 & -222149.4 & 18.7 & 8.9 & 105 & $<16.5$ & $494(170)$ & $159(90)$ \\
\hline 1314 & G007.632-00.110 & 180312.8 & -222428.3 & 180311.9 & -222433.1 & 29.7 & 15.8 & 40 & 33.26 & $1340(190)$ & $1450(150)$ \\
\hline 1316 & G007.636-00.194 & 180331.5 & -222645.8 & 180331.4 & -222649.4 & 18.0 & 14.9 & 77 & 18.37 & $617(140)$ & $595(94)$ \\
\hline 1317 & G007.650-00.166 & 180327.6 & -222513.3 & 180326.8 & -222515.9 & 24.3 & 17.6 & 35 & 34.95 & 735 (150) & $562(120)$ \\
\hline 1320 & G007.762+00.076 & 180248.1 & -221209.8 & 180246.5 & -221216.0 & 29.1 & 19.6 & 15 & 44.81 & $584(180)$ & $565(170)$ \\
\hline 1322 & G007.898-00.012 & 180323.9 & -220742.2 & 180323.7 & -220745.9 & 29.1 & 14.7 & 173 & 25.12 & $615(170)$ & $552(130)$ \\
\hline
\end{tabular}

Notes. Errors are given in parentheses.

(This table is available in its entirety in a machine-readable form in the online journal. A portion is shown here for guidance regarding its form and content.)

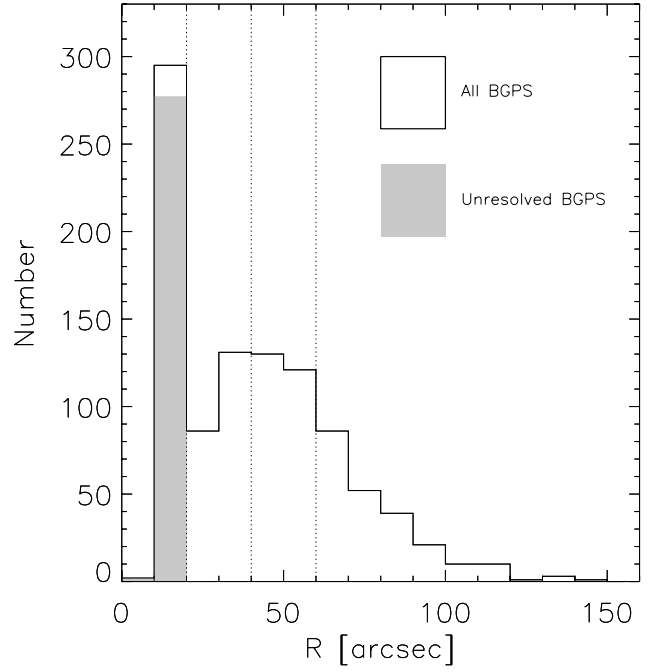

Figure 1. Distribution of angular source sizes $(R)$ for the 631 BGPS sources that fall within the selected ranges (see Section 2). The white histogram denotes all source sizes, the gray histogram denotes sources that are unresolved compared to the BGPS effective beam size (FWHM of $33^{\prime \prime}, R=16^{\prime \prime} .5$ ), and the dotted lines mark the radii of the apertures presented in the BGPS catalog.

where $\sigma_{\text {beam }}=\Theta_{\mathrm{FWHM}} / \sqrt{8 \ln 2}=14^{\prime \prime}, \Theta_{\mathrm{FWHM}}=33^{\prime \prime}$, and $\eta$ is a factor relating the axis dispersions to the true size of the source. $\eta=2.4$ is adopted as the median value derived from measuring the observed major and minor axes compared with the true radius for a variety of simulated emission profiles spanning a range of density distributions, sizes relative to the beam and signal-to-noise ratios (Rosolowsky et al. 2010). Including $\eta$ provides an effective radius that corresponds to the full extent of the emission detected by the BGPS. Specifically, $R$ describes the radius over which $S_{v}$ (int) was calculated. Due to the cleaning methods employed (PCA cleaning; see Aguirre et al. 2011), the BGPS resolves out uniform structure on size scales larger than 5.9. Thus, the BGPS is sensitive to structures with diameters between $33^{\prime \prime}$ and 5.9.

In order to sample source properties across a large range of Galactocentric radius $\left(R_{\mathrm{Gal}}\right)$, we have obtained $\mathrm{NH}_{3}$ and $\mathrm{H}_{2} \mathrm{O}$ spectral line observations toward 631 BGPS sources within four distinct Galactic longitude ranges: $7.5 \leqslant \ell \leqslant 10.0$, $19.5 \leqslant \ell \leqslant 22.5,31.3 \leqslant \ell \leqslant 34.5$, and $52.5 \leqslant \ell \leqslant 55^{\circ} .5$. Sources near $\ell=33^{\circ}$ were targeted because of the peak in millimeter continuum sources along that line of sight, which corresponds to the end of the Galactic bar and tangent of the Scutum spiral arm. The $\ell \sim 54^{\circ}$ field was chosen to sample the tangent of the Sagittarius Arm. The $\ell \sim 9^{\circ}$ and $\ell \sim 20^{\circ}$ fields were chosen to sample sources with small $R_{\mathrm{Gal}}$. Table 1 presents the 631 BGPS sources that lie within our Galactic longitude ranges: BGPS source catalog number (Column 1), source name (Column 2), centroid R.A. and decl. (Columns 3 and 4), R.A. and decl. of the peak emission (Columns 5 and 6), major and minor axes (Columns 7 and 8), position angle north of $\ell=0^{\circ}$ (Column 9), radius (Column 10), and $S_{\nu}\left(120^{\prime \prime}\right)$ and $S_{\nu}$ (int) (Columns 11 and 12).

We have chosen to use $S_{\nu}\left(120^{\prime \prime}\right)$ as well as $S_{\nu}$ (int) due to their complementary nature. $S_{v}\left(120^{\prime \prime}\right)$ is a well-defined quantity that is easily compared with other data sets but may not represent the true source flux due to source crowding and/or irregularly shaped, large sources. $S_{v}$ (int) is difficult to reproduce and compare with other data sets since it is a product of the BGPS source extraction algorithm. However, $S_{v}$ (int) will more accurately represent the source flux density.

When choosing which aperture size to use, we considered the distribution of source sizes as well as the distribution of angular separation between BGPS sources. The distribution of angular source sizes is shown in Figure 1 where the white histogram represents the angular source size of all 631 BGPS sources, and the gray histogram denotes sources unresolved compared to the beam. The dotted lines mark the three aperture sizes included in Bolocat. The $120^{\prime \prime}$ aperture is larger than most sources and will therefore underestimate the source flux in the smallest number of BGPS sources, although the number of sources larger than the $120^{\prime \prime}$ aperture is not negligible. Figure 2 shows the distribution of the angular distance to the nearest BGPS source. This distribution favors the smaller aperture sizes since they will suffer the least from source contamination. We must balance the effects of missing flux and including additional flux when choosing an aperture size. As demonstrated in Figure 2, 217 BGPS sources have at least one source closer than $120^{\prime \prime}$. We have chosen to use the $120^{\prime \prime}$ aperture even though the flux will be overestimated in 217 of the 631 BGPS sources within our longitude ranges. $S_{v}\left(120^{\prime \prime}\right)$ will, on average, overestimate $S_{v}$ (int) by $10 \%$; the mean ratio of $S_{v}\left(120^{\prime \prime}\right)$ to $S_{v}$ (int) is 1.09 with a standard deviation of 0.50 . Therefore, we present $S_{v}\left(120^{\prime \prime}\right)$ as a comparison point for other studies, while our analysis is based on $S_{v}$ (int), which will not suffer from the two problems discussed. We stress that $S_{v}\left(120^{\prime \prime}\right)$ will overestimate the fluxes 


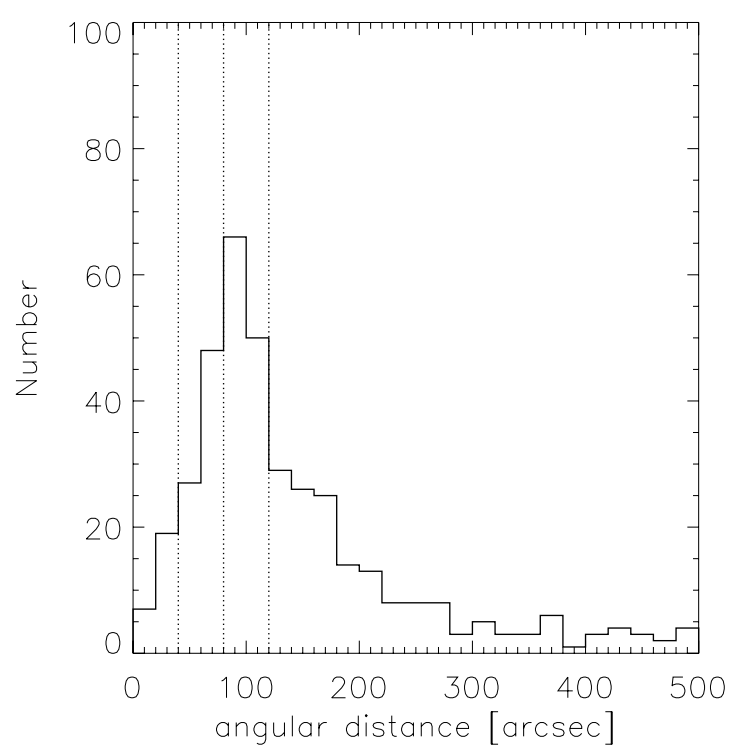

Figure 2. Distribution of angular distance to the nearest BGPS source. The dotted lines represent the diameters of the apertures presented in the BGPS catalog.

Table 2

$\mathrm{NH}_{3}$ Observation Dates and Atmospheric Opacities

\begin{tabular}{llc}
\hline \hline Session & \multicolumn{1}{c}{ Date } & $\tau_{23}{ }^{\mathrm{a}}$ \\
\hline 1 & 2008 Feb 14 & 0.047 \\
2 & 2008 Feb 16 & 0.085 \\
3 & 2008 Mar 23 & 0.046 \\
4 & 2010 Jan 10 & 0.031 \\
5 & 2010 Jan 14 & 0.043 \\
6 & 2010 Jan 31 & 0.036 \\
7 & 2010 Feb 7 & 0.039 \\
8 & 2010 Feb 11 & 0.052 \\
9 & 2010 Feb 20 & 0.055 \\
10 & 2010 Mar 6 & 0.041 \\
11 & 2010 Mar 9 & 0.059 \\
12 & 2010 Mar 27 & 0.039 \\
RMS & 2010 Feb 13 & 0.054 \\
RMS & 2010 Sep 25 & 0.120 \\
\hline
\end{tabular}

Note. ${ }^{\text {a }}$ Atmospheric opacity at $23 \mathrm{GHz}$.

to approximately one-third of BGPS sources included in this paper.

We additionally consider $S_{v}\left(40^{\prime \prime}\right)$ as a representative of the millimeter flux per beam since the solid angle of the $40^{\prime \prime}$ aperture $\left(2.95 \times 10^{-8} \mathrm{sr}\right)$ is equivalent to the solid angle subtended by the effective Gaussian beam of the BGPS $\left(2.9 \times 10^{-8} \mathrm{sr}\right) . S_{v}\left(40^{\prime \prime}\right)$ is only employed when calculating the $\mathrm{H}_{2}$ column density per beam and $\mathrm{NH}_{3}$ abundance (see Section 6.7).

\section{3. $\mathrm{NH}_{3}$ SURVEY OF BGPS SOURCES}

We have conducted spectroscopic follow-up observations of the 631 BGPS sources selected as described in the previous section. We observed all sources in the lowest inversion transitions of $\mathrm{NH}_{3}$ and the $22 \mathrm{GHz}$ water maser transition using the Robert F. Byrd Green Bank Telescope ${ }^{9}$ (GBT). The dates of observations and $23 \mathrm{GHz}$ zenith opacities are listed in Table 2.

\footnotetext{
9 The GBT is operated by the National Radio Astronomy Observatory, which is a facility of the National Science Foundation, operated under cooperative agreement by Associated Universities, Inc.
}

Table 3

Spectral Line Observations

\begin{tabular}{lccc}
\hline \hline Line & $\begin{array}{c}v \\
(\mathrm{GHz})\end{array}$ & $\begin{array}{c}\text { Mean RMS } \\
(\mathrm{mK})\end{array}$ & $\begin{array}{c}\text { Stdev RMS } \\
(\mathrm{mK})\end{array}$ \\
\hline $\mathrm{NH}_{3}(1,1)$ & 23.6944961 & 98 & 28 \\
$\mathrm{NH}_{3}(2,2)$ & 23.7226328 & 93 & 27 \\
$\mathrm{NH}_{3}(3,3)$ & 23.8701270 & 96 & 26 \\
$\mathrm{NH}_{3}(4,4)$ & 24.1394160 & 141 & 33 \\
$\mathrm{H}_{2} \mathrm{O}$ & 22.2350801 & 87 & 32 \\
\hline
\end{tabular}

The observations consisted of two-minute integrations on each source, with pointing and focus checks approximately every $1.5 \mathrm{hr}$. We additionally checked the flux calibration by periodically injecting a noise signal through observations of 3C309.1, a National Radio Astronomy Observatory (NRAO) flux calibrator with a known flux. We repeated this flux calibration observation during all sessions except for sessions lasting two hours or less (sessions 4 and 11). We did not observe any significant variation throughout the measurements $(<10 \%)$, and use a mean calibration for all observations.

The GBT spectrometer served as the back end and was configured to observe four intermediate frequencies (IFs). The majority of sources were observed with the following four lines: $22 \mathrm{GHz} \mathrm{H}_{2} \mathrm{O}, \mathrm{NH}_{3}(1,1), \mathrm{NH}_{3}(2,2)$, and $\mathrm{NH}_{3}(3,3)$. Thirty of the spectra were observed with an identical setup by the RMS team, who were kind enough to share their raw data. The sources observed during 2008 were observed with the $\mathrm{NH}_{3}(4,4)$ in place of $\mathrm{NH}_{3}(3,3)$. The frequencies of each line are listed in Table 3. We utilized frequency switching with a $5 \mathrm{MHz}$ throw, and the RMS team used a throw of $8 \mathrm{MHz}$. At these frequencies, the GBT FWHM is $31^{\prime \prime}$ and is well matched to the effective BGPS FWHM beam size of $33^{\prime \prime}$.

We reduced the spectral line data using the GBTIDL reduction package. We extracted each scan, and folded it to account for the effects of frequency switching. In order to place the data on the $T_{A}$ scale, we calibrated the noise diodes using the observations of sources with known fluxes. We then subtracted a first-order baseline (second order for the $\mathrm{H}_{2} \mathrm{O}$ spectra), and converted the spectra to the $T_{A}^{*}$ scale by accounting for the atmospheric opacity. We then divided by the GBT main beam efficiency, $\eta \sim 0.81$ at $23 \mathrm{GHz}$, to place the spectra on the $T_{\mathrm{mb}}$ scale. Each polarization was calibrated separately then averaged together, as were multiple observations when applicable. The mean rms and standard deviation of the rms for each possible IF are listed in Table 3.

Figure 3 shows the observed spectra for BGPS source 1322, including $\mathrm{NH}_{3}(1,1),(2,2),(3,3)$, and the $22 \mathrm{GHz} \mathrm{H}_{2} \mathrm{O}$ maser transition. The green line overplotted in each panel displays the model fit described in Section 3.

The parameter estimation follows the philosophy developed by Rosolowsky et al. (2008) and adapted for use in D10. The method models the emission as a beam filling slab of ammonia with a variable column density $\left(N_{\mathrm{NH} 3}\right)$, kinetic temperature $\left(T_{\mathrm{K}}\right)$, excitation temperature $\left(T_{\mathrm{ex}}\right)$, Gaussian line width $\left(\sigma_{\mathrm{Vlsr}}\right)$, and LSR velocity $\left(v_{\mathrm{LSR}}\right)$. Previous versions of the pipeline used the optical depth of the $(1,1)$ line as a free parameter and then calculated the column density. This model reverses the direction of the calculation, assuming that the molecules are in thermodynamic equilibrium using an ortho-to-para ratio of 1:1, which is the high temperature formation limit (Takano et al. 2002). Hence, the ammonia molecules are partitioned among 

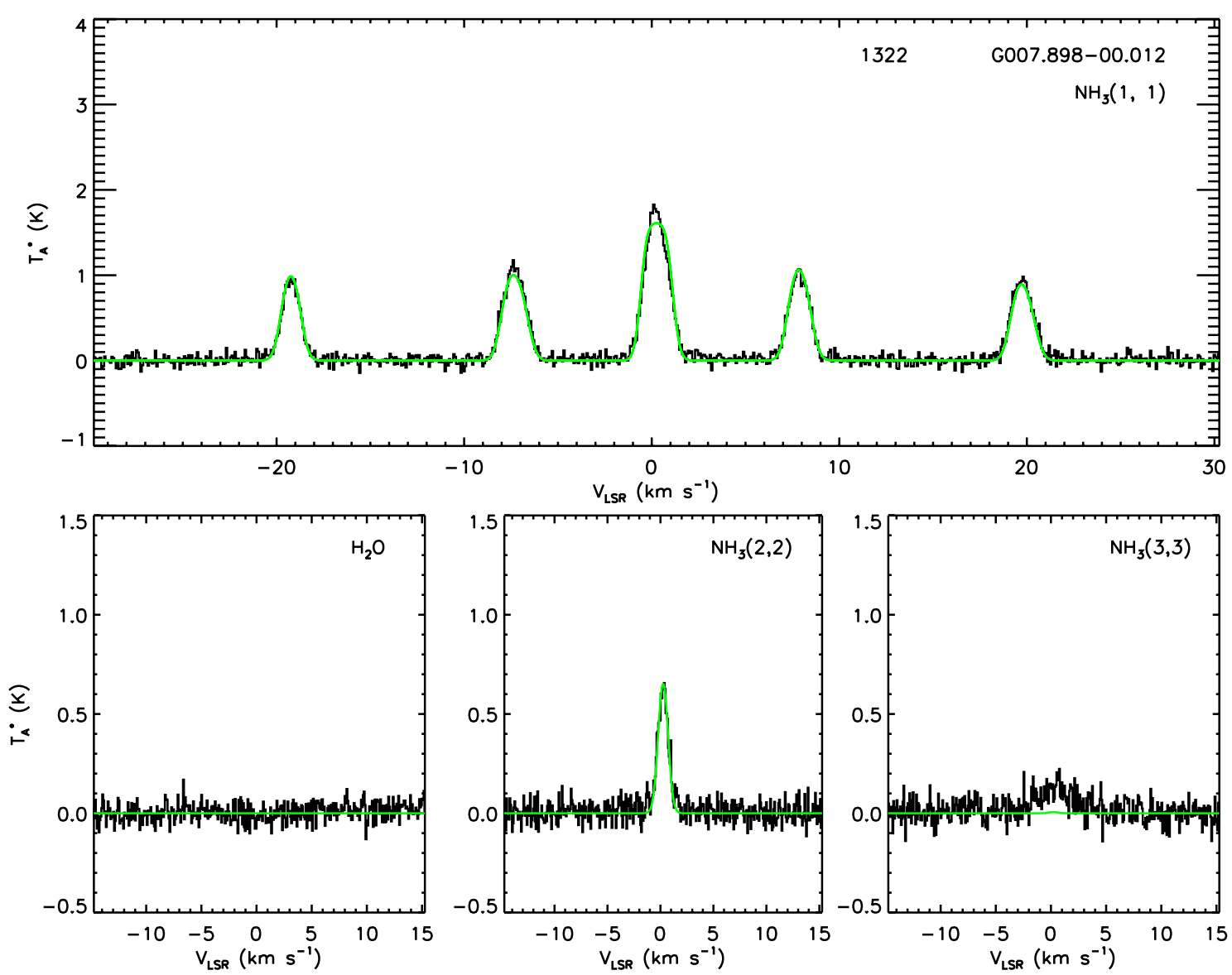

Figure 3. Spectra toward BGPS source 1322. Upper panel: $\mathrm{NH}_{3}(1,1)$. Lower panel from left to right: $22 \mathrm{GHz} \mathrm{H}_{2} \mathrm{O}$ maser transition, $\mathrm{NH}_{3}(2,2)$, and $\mathrm{NH}_{3}(3,3)$. The green lines show the model fit to the observed spectra. Similar figures for the $514 \mathrm{NH}_{3}$ pointings detected in the $(1,1)$ line (see Section 4$)$ are included as an online-only figure.

(The complete figure set (514 images), color version of this figure is available in the online journal.)

the energy levels as

$$
\begin{aligned}
Z_{O}=1 & +\sum_{J, K, i} 2(2 J+1) \\
& \times \exp \left\{-\frac{h\left[B J(J+1)+(C-B) J^{2}\right]+\Delta E(J, K, i)}{k T_{k}}\right\}
\end{aligned}
$$$$
\text { for } J=3,6,9, \ldots ; i=0,1 \text {, }
$$

$$
\begin{aligned}
Z_{P}= & \sum_{J, K, i}(2 J+1) \\
& \times \exp \left\{-\frac{h\left[B J(J+1)+(C-B) J^{2}\right]+\Delta E(J, K, i)}{k T_{k}}\right\}
\end{aligned}
$$

for $J=1,2,4,5, \ldots ; i=0,1$,

where $Z_{O}$ and $Z_{P}$ correspond to ortho and para $\mathrm{NH}_{3}$, respectively. Here, $\Delta E(J, K, i)$ is the energy difference due to the splitting of the symmetric and anti-symmetric states. $\Delta E(J, K, 1)$ corresponds to the antisymmetric state, which is $\Delta E / k \sim 1.1 \mathrm{~K}$ above the symmetric state $(\Delta E(J, K, 0)=0)$. The column density of the molecules in the $N_{\mathrm{NH} 3}(J, K, i)$ state is thus $N_{\mathrm{NH} 3} Z_{O}(J, K, i) /\left(2 Z_{O}\right)$ and $N_{\mathrm{NH} 3} Z_{P}(J, K, i) /\left(2 Z_{P}\right)$, where the factor of two in the denominator accounts for our assumption of ortho:para $=1: 1$. From the column densities in the indi- vidual states, we calculate the optical depths in the individual transitions. From here, the method follows that of previous work: we use the optical depth, hyperfine structure, the velocity information, and the excitation conditions to model the individual spectra. The free parameters are optimized using the MPFIT least-squares minimization routine including parameter bounds (Markwardt 2009).

These small changes do produce differences in the derived parameters, reflecting optimizing slightly different functions. In addition, the optimization weights the lines slightly differently under the different schemes. The variation largely reflects the approximate nature of the assumed model, namely that of a homogeneous slab with uniform properties. While more sophisticated models could produce better fits, they would require additional information such as mapping to constrain the distribution of the individual lines. As an example, consider the fit to an optically thin object which has a temperature that decreases with radius. In this case, the model would underestimate the $(3,3)$ line emission by a noticeable margin. This is because the temperature fit is largely driven by the bright $(1,1)$ and $(2,2)$ lines, which can be excited throughout the object. The $(3,3)$ line could show significant excitation in the center of the object but this emission would be underestimated since the temperature parameter is largely determined by the strong emission from the other two lines. Furthermore, variations in the ortho-to-para ratios could affect the fits; however, we would require substantially more information to resolve these variations. The parameter fits 


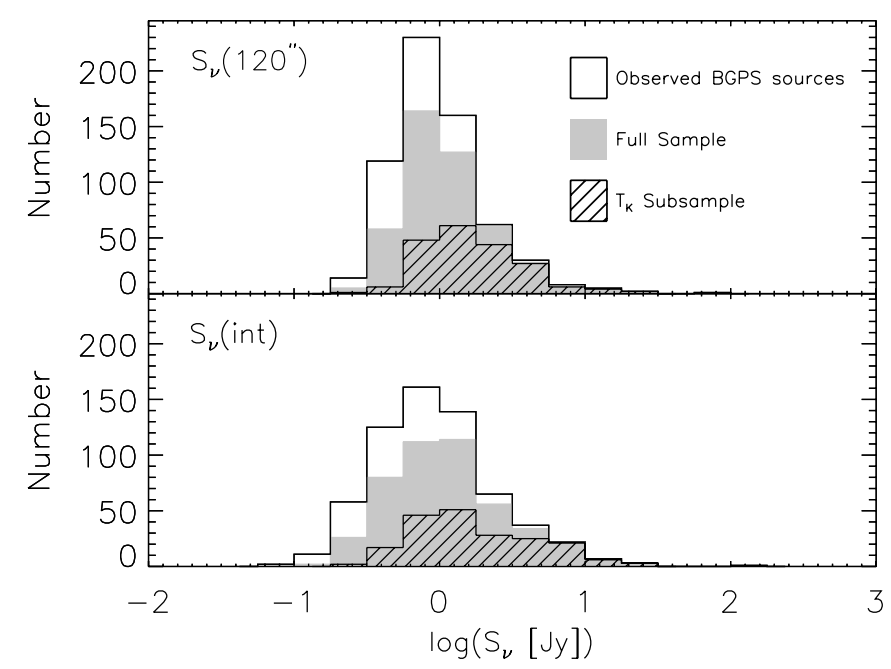

Figure 4. Distributions of $S_{v}\left(120^{\prime \prime}\right)$ (top panel) and $S_{v}$ (int) (bottom panel). The white histograms include all BGPS sources observed in $\mathrm{NH}_{3}$, the gray histograms include the full sample, and the striped histograms include the $T_{\mathrm{K}}$ subsample.

represent emission-weighted averages of the parameters under consideration.

The changes to the parameter estimation have resulted in approximately $10 \%$ changes in the $\mathrm{NH}_{3}$-based parameters. For example, the mean $T_{\mathrm{K}}$ of the sources in the Gemini OB1 molecular cloud has decreased by roughly $2 \mathrm{~K}$ from the values presented in D10. Because of the changes, we have reanalyzed the Gem OB1 sources as well and present the results from the newest pipeline in the comparison in this work.

\section{RESULTS}

Of the $737 \mathrm{NH}_{3}$ pointings, 722 overlap with BGPS sources in the v1.0 catalog, while the remaining 15 pointings were selected by eye from an earlier BGPS catalog. The 15 unmatched pointings are excluded from our analysis because they do not have corresponding $1.1 \mathrm{~mm}$ data. Eight of the fifteen unmatched sources do have a detection in the $\mathrm{NH}_{3}(1,1)$ line, suggesting that some of the low intensity BGPS emission is likely real. We have additionally excluded 10 of the $737 \mathrm{NH}_{3}$ pointings due to radio frequency interference (RFI) in the $\mathrm{NH}_{3}(2,2) \mathrm{IF}$. Six of the ten pointings with RFI were detected in the $\mathrm{NH}_{3}(1,1)$ transition, but we are unable to characterize the gas properties without a realistic upper limit for the $(2,2)$ line.

To detect a line, we require $W \geqslant 5 \Delta \mathrm{W}$ and $T_{\mathrm{pk}} \geqslant 4 \sigma$, where $W$ is the integrated intensity, $\Delta W$ is the error in integrated intensity, and $T_{\mathrm{pk}}$ is the peak brightness temperature of the line. We have detected the $\mathrm{NH}_{3}(1,1)$ line in 514 of the $707 \mathrm{NH}_{3}$ pointings that were matched with a BGPS source and were not affected by RFI, for a detection rate of $73 \%$. Since the positions of the observations obtained in 2008 were determined by eye from early-BGPS data products there are multiple $\mathrm{NH}_{3}$ pointings per BGPS source in some cases. The $514 \mathrm{NH}_{3}$ pointings detected in the $(1,1)$ line correspond to 456 unique BGPS sources ( $72 \%$ of the 631 BGPS sources observed).

Table 4 lists the observed $\mathrm{NH}_{3}$ properties for the 514 pointings detected in the $(1,1)$ line, including BGPS source number (Column 1), R.A. and decl. of the $\mathrm{NH}_{3}$ pointing (Columns 2 and 3$)$, radial velocity $\left(V_{\mathrm{LSR}}\right.$; Column 4$)$, velocity dispersion $\left(\sigma_{\mathrm{Vlsr}} ;\right.$ Column 5$)$, and peak main beam temperature $\left(T_{\mathrm{mb}}\right)$ and integrated intensity $(W)$ for the $(1,1),(2,2),(3,3)$, and $(4,4)$

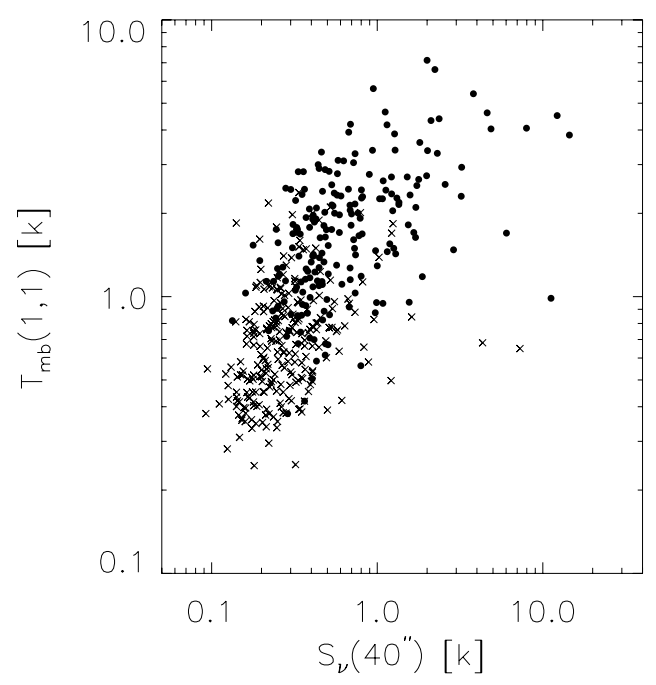

Figure 5. Observed peak $T_{\mathrm{mb}}$ of the $\mathrm{NH}_{3}(1,1)$ line vs. the flux within a beam, $S_{v}\left(40^{\prime \prime}\right)$. Circles mark sources with a detection in both the $(1,1)$ and $(2,2)$ lines while crosses mark sources with only an upper limit in the $(2,2)$ line.

$\mathrm{NH}_{3}$ transitions (Columns 6-13). Lines that were observed but not detected are listed as upper-limits based on the observed rms $(\sigma)$, and are given by $T_{\mathrm{mb}}<4 \sigma$ and $W<5 \Delta W$, where $\Delta W=\sigma \delta V \sqrt{N}$ is the error in the integrated intensity, $\delta V$ is the channel width in velocity, and $N$ is the number of channels over which the rms was calculated. These 514 spectra are included in an online version of Figure 3.

In the following analysis, we consider only the $\mathrm{NH}_{3}$ pointings that lie closest to the $1.1 \mathrm{~mm}$ peaks and refer to the BGPS sources and these pointings as the "full sample." We additionally define two subsamples from the full sample: the " $T_{\mathrm{K}}$ subsample" and the "near subsample." The $T_{\mathrm{K}}$ subsample consists of 199 BGPS sources that were detected in the $(2,2)$ line. Since sources without a $(2,2)$ detection can only provide an upper limit for the gas kinetic temperature, the $T_{\mathrm{K}}$ subsample is used throughout this section and Section 6 to characterize the properties of sources excluding sources that only have upper limits. The near subsample consists of 199 BGPS sources that were placed at the near kinematic distance (see Section 5) and is used in Section 7 to assess the trends in source properties as a function of position within the Galaxy.

The distribution of $1.1 \mathrm{~mm}$ flux densities is shown in Figure 4. We present flux densities within both a well-defined aperture $\left(S_{v}\left(120^{\prime \prime}\right)\right.$; top panel) and the integrated flux density, which is dependent on the reduction and source extraction algorithms ( $S_{v}$ (int); bottom panel). The white histograms denote the distribution of all observed BGPS sources, the gray histograms denote the full sample, and the striped histograms denote the $T_{\mathrm{K}}$ subsample. The high flux density end of the distribution is represented well in both the full sample and the subsample, while a significant fraction of the faint BGPS sources are lacking $(1,1)$ and $(2,2)$ detections.

Figure 5 plots $T_{\mathrm{mb}}(1,1)$ versus the $1.1 \mathrm{~mm}$ flux per beam, $S_{v}\left(40^{\prime \prime}\right)$, for the full sample. The $T_{\mathrm{K}}$ subset is shown as black circles, while sources without a detection in the $(2,2)$ line are shown as crosses. As expected, the $(2,2)$ line was not detected in pointings toward the weaker $1.1 \mathrm{~mm}$ sources with faint $(1,1)$ lines.

The observed radial velocities for the full sample are shown in Figure 6. The black circles denote the $T_{\mathrm{K}}$ subsample and the crosses mark BGPS sources without a $(2,2)$ detection. 
Table 4

Observed $\mathrm{NH}_{3}$ Properties

\begin{tabular}{|c|c|c|c|c|c|c|c|c|c|c|c|c|}
\hline $\begin{array}{l}\text { ID } \\
\text { Number }^{\mathrm{a}}\end{array}$ & $\begin{array}{l}\text { R.A. } \\
\text { (J2000) }\end{array}$ & $\begin{array}{l}\text { Decl. } \\
(\text { J2000) }\end{array}$ & $\begin{array}{c}V_{\mathrm{LSR}} \\
\left(\mathrm{km} \mathrm{s}^{-1}\right)\end{array}$ & $\begin{array}{c}\sigma_{\mathrm{Vlsr}} \\
\left(\mathrm{km} \mathrm{s}^{-1}\right)\end{array}$ & $\begin{array}{c}T_{\mathrm{mb}}(1,1) \\
(\mathrm{K})\end{array}$ & $\begin{array}{c}W(1,1) \\
\left(\mathrm{K} \mathrm{km} \mathrm{s}^{-1}\right)\end{array}$ & $\begin{array}{c}T_{\mathrm{mb}}(2,2)^{\mathrm{b}} \\
(\mathrm{K})\end{array}$ & $\begin{array}{c}W(2,2)^{\mathrm{b}, \mathrm{c}} \\
\left(\mathrm{K} \mathrm{km} \mathrm{s}^{-1}\right)\end{array}$ & $\begin{array}{c}T_{\mathrm{mb}}(3,3)^{\mathrm{b}, \mathrm{c}} \\
(\mathrm{K})\end{array}$ & $\begin{array}{c}W(3,3)^{\mathrm{b}, \mathrm{c}} \\
\left(\mathrm{K} \mathrm{km} \mathrm{s}^{-1}\right)\end{array}$ & $\begin{array}{c}T_{\mathrm{mb}}(4,4)^{\mathrm{b}, \mathrm{c}} \\
(\mathrm{K})\end{array}$ & $\begin{array}{c}W(4,4)^{\mathrm{b}, \mathrm{c}} \\
\left(\mathrm{K} \mathrm{km} \mathrm{s}^{-1}\right)\end{array}$ \\
\hline 1307 & 180230.0 & -222806.9 & $32.63(0.03)$ & $0.94(0.03)$ & $0.85(0.10)$ & $6.08(0.20)$ & $<0.35$ & $<0.97$ & $<0.39$ & $<0.95$ & $\ldots$ & $\cdots$ \\
\hline 1309 & 180100.5 & -221548.5 & $8.16(0.06)$ & $0.94(0.06)$ & $0.39(0.09)$ & $3.21(0.18)$ & $<0.35$ & $<0.97$ & $<0.39$ & $<0.98$ & $\ldots$ & $\cdots$ \\
\hline 1310 & 180247.8 & -222603.3 & $122.87(0.03)$ & $0.60(0.02)$ & $0.75(0.09)$ & $4.08(0.18)$ & $<0.35$ & $<0.86$ & $<0.38$ & $<0.83$ & $\ldots$ & $\cdots$ \\
\hline 1311 & 180315.0 & -222709.7 & $153.30(0.01)$ & $0.80(0.01)$ & $2.54(0.10)$ & $19.31(0.21)$ & $1.38(0.09)$ & $5.88(0.19)$ & $1.02(0.10)$ & $7.09(0.19)$ & $\ldots$ & $\cdots$ \\
\hline 1313 & 180245.3 & -222146.8 & $-0.71(0.04)$ & $0.77(0.04)$ & $0.63(0.09)$ & $3.94(0.19)$ & $<0.35$ & $<0.92$ & $<0.37$ & $<0.88$ & $\ldots$ & $\ldots$ \\
\hline 1314 & 180311.9 & -222432.5 & $154.02(0.01)$ & $0.97(0.01)$ & $3.38(0.11)$ & $28.53(0.23)$ & $2.07(0.09)$ & $10.59(0.20)$ & $1.60(0.10)$ & $12.32(0.20)$ & $\ldots$ & $\cdots$ \\
\hline 1316 & 180331.5 & -222648.4 & $152.52(0.00)$ & $0.63(0.00)$ & $3.93(0.10)$ & $24.58(0.20)$ & $2.09(0.09)$ & $7.47(0.18)$ & $1.19(0.09)$ & $6.53(0.17)$ & $\cdots$ & $\cdots$ \\
\hline 1317 & 180326.8 & -222516.1 & $154.55(0.02)$ & $1.01(0.02)$ & $1.26(0.10)$ & $10.45(0.21)$ & $0.48(0.09)$ & $2.49(0.20)$ & $<0.38$ & $<0.96$ & $\cdots$ & $\cdots$ \\
\hline 1320 & 180246.6 & -221217.7 & $18.11(0.03)$ & $0.76(0.03)$ & $0.75(0.09)$ & $4.24(0.18)$ & $<0.34$ & $<0.90$ & $<0.38$ & $<0.89$ & $\ldots$ & $\cdots$ \\
\hline 1322 & 180323.7 & -220745.9 & $0.26(0.00)$ & $0.42(0.00)$ & $3.00(0.09)$ & $15.52(0.16)$ & $1.08(0.09)$ & $2.12(0.15)$ & $<0.38$ & $<0.74$ & $\cdots$ & $\cdots$ \\
\hline
\end{tabular}

Notes. Errors are given in parentheses.

${ }^{\mathrm{a}} \mathrm{B}, \mathrm{C}, \mathrm{D}$, and $\mathrm{E}$ denote multiple ammonia pointings that fall within a single $1.1 \mathrm{~mm}$ source.

${ }^{\mathrm{b}}$ Upper limits are $T_{\mathrm{mb}}<4 \sigma$ and $W<5 \sigma \Delta v \sqrt{N}$, where $\sigma$ is the rms noise, $\Delta v$ is the width of a single channel in velocity, and $N$ is the number of pixels over which the average RMS was calculated.

c $\ldots$ denotes sources that were not observed in the given transition.

(This table is available in its entirety in a machine-readable form in the online journal. A portion is shown here for guidance regarding its form and content.) 
Table 5

Derived Gas Properties

\begin{tabular}{|c|c|c|c|c|c|c|c|}
\hline $\begin{array}{l}\text { ID } \\
\text { Number }^{\mathrm{a}}\end{array}$ & $\tau(1,1)$ & $\begin{array}{l}T_{\mathrm{K}}^{\mathrm{b}} \\
(\mathrm{K})\end{array}$ & $\begin{array}{l}T_{\mathrm{ex}} \\
(\mathrm{K})\end{array}$ & $\begin{array}{c}a \\
\left(\mathrm{~km} \mathrm{~s}^{-1}\right)\end{array}$ & $\begin{array}{c}\sigma_{\mathrm{NT}} \\
\left(\mathrm{km} \mathrm{s}^{-1}\right)\end{array}$ & $\begin{array}{c}\mathrm{H}_{2} \mathrm{O} \\
\text { Maser? }\end{array}$ & $\begin{array}{l}\text { Multiple } \\
\text { Comp? }\end{array}$ \\
\hline 1307 & 4.31 & $<11.90(0.47)$ & $3.29(0.13)$ & $0.20(0.002)$ & $0.93(0.06)$ & $\mathrm{Y}$ & \\
\hline 1309 & 2.70 & $<28.59(1.17)$ & $3.01(0.13)$ & $0.32(0.004)$ & $0.93(0.11)$ & Y & \\
\hline 1310 & 4.31 & $<12.61(0.46)$ & $3.27(0.11)$ & $0.21(0.002)$ & $0.59(0.05)$ & $\mathrm{N}$ & \\
\hline 1311 & 6.06 & $24.29(0.19)$ & $4.03(0.04)$ & $0.29(0.001)$ & $0.80(0.02)$ & $\mathrm{Y}$ & $v_{\mathrm{lsr}}$ \\
\hline 1313 & 2.79 & $<14.00(0.75)$ & $3.24(0.17)$ & $0.22(0.003)$ & $0.77(0.08)$ & $\mathrm{N}$ & \\
\hline 1314 & 5.12 & $27.44(0.13)$ & $4.60(0.03)$ & $0.31(0.000)$ & $0.96(0.01)$ & $\mathrm{Y}$ & $T_{\mathrm{K}}$ \\
\hline 1316 & 5.15 & $18.01(0.10)$ & $5.51(0.04)$ & $0.25(0.000)$ & $0.63(0.01)$ & $\mathrm{Y}$ & $T_{\mathrm{K}}$ \\
\hline 1317 & 3.62 & $14.40(0.23)$ & $3.72(0.06)$ & $0.22(0.001)$ & $1.01(0.04)$ & $\mathrm{N}$ & \\
\hline 1320 & 2.14 & $<15.76(0.68)$ & $3.42(0.15)$ & $0.23(0.002)$ & $0.76(0.07)$ & $\mathrm{Y}$ & \\
\hline 1322 & 8.34 & $12.38(0.12)$ & $4.79(0.05)$ & $0.21(0.000)$ & $0.41(0.01)$ & $\mathrm{N}$ & $T_{\mathrm{K}}$ \\
\hline
\end{tabular}

Notes. Errors are given in parentheses.

${ }^{\mathrm{a}} \mathrm{B}, \mathrm{C}, \mathrm{D}$, and $\mathrm{E}$ denote multiple ammonia pointings that fall within a single $1.1 \mathrm{~mm}$ source.

${ }^{\mathrm{b}}$ Upper limits to $T_{\text {kin }}$ are due to a non-detection of the $\mathrm{NH}_{3}(2,2)$ transition.

${ }^{\mathrm{c}} \mathrm{Y}$ denotes detected, $\mathrm{N}$ denotes not detected.

${ }^{\mathrm{d}} T_{\mathrm{K}}$ denotes additional warm temperature component might provide a better fit, and $v_{\mathrm{lsr}}$ denotes multiple velocity components required.

(This table is available in its entirety in a machine-readable form in the online journal. A portion is shown here for guidance regarding its form and content.)

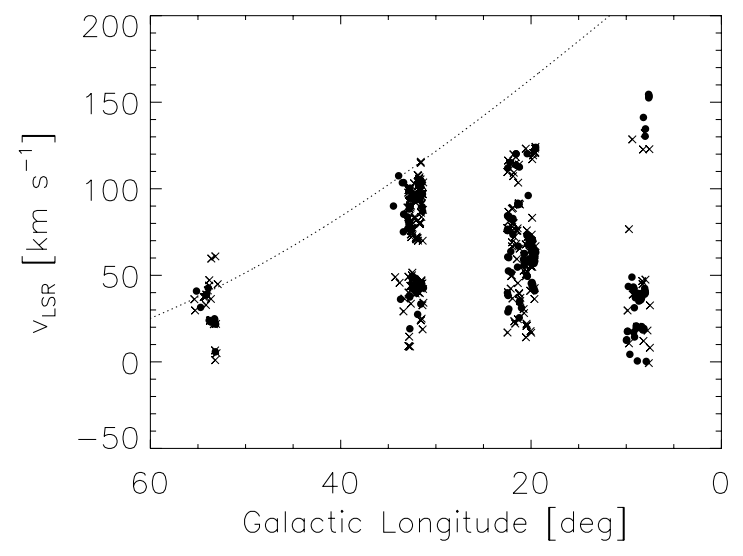

Figure 6. Observed $v_{\text {lsr }}$ for the full sample. Black circles mark the $T_{\mathrm{K}}$ subsample, while crosses mark sources without a $(2,2)$ detection. The dotted line denotes the maximum radial velocity expected from circular rotation given by $v_{\text {lsr, } \max }=\Theta\left(R_{o}\right)(1-\sin (\ell))$. Sources with radial velocities in excess of $v_{\text {lss, max }}$ are placed at the tangent distance.

The dotted line denotes the maximum radial velocity expected from Galactic circular rotation as a function of $\ell$ given by $v_{\text {lsr, } \max }=\Theta\left(R_{o}\right)(1-\sin (\ell))$, where $\Theta\left(R_{o}\right)$ is the orbital velocity of the Sun about the Galactic center. The observed radial velocities follow the distribution of ${ }^{13} \mathrm{CO}(J=1-0)$ seen in the BU-FCRAO GRS (Jackson et al. 2006). There are 24 sources that show some evidence of two velocity components. These sources are noted in Column 8 of Table 5.

We also measure the Gaussian width of the $(1,1)$ line, $\sigma_{\mathrm{Vlsr}}$, and plot the distribution of the full sample (gray histogram) and the $T_{\mathrm{K}}$ subsample (striped histogram) in Figure 7. Note that $\sigma_{\mathrm{Vlsr}}=\mathrm{FWHM} / \sqrt{8 \ln 2}$. The dashed line denotes the spectral resolution of the $\mathrm{NH}_{3}$ observations of $0.08 \mathrm{~km} \mathrm{~s}^{-1}$. The full sample can be characterized by $\left\langle\sigma_{\mathrm{Vlsr}}\right\rangle=0.76 \pm 0.49 \mathrm{~km} \mathrm{~s}^{-1}$ and the $T_{\mathrm{K}}$ subsample by $\left\langle\sigma_{\mathrm{Vlsr}}\right\rangle=0.81 \pm 0.53 \mathrm{~km} \mathrm{~s}^{-1}$. We observe line widths up to $\sigma_{\mathrm{Vlsr}} \sim 4 \mathrm{~km} \mathrm{~s}^{-1}$. Of the 11 sources with $\sigma_{\mathrm{Vlsr}} \geqslant 2 \mathrm{~km} \mathrm{~s}^{-1}, 6$ are weak detections with poor model fits, 1 has two velocity components, and 4 truly do exhibit line widths greater than $2 \mathrm{~km} \mathrm{~s}^{-1}$. See Table 8 for statistical properties of $\sigma_{\mathrm{Vlsr}}$ for both the full sample and the $T_{\mathrm{K}}$ subsample.

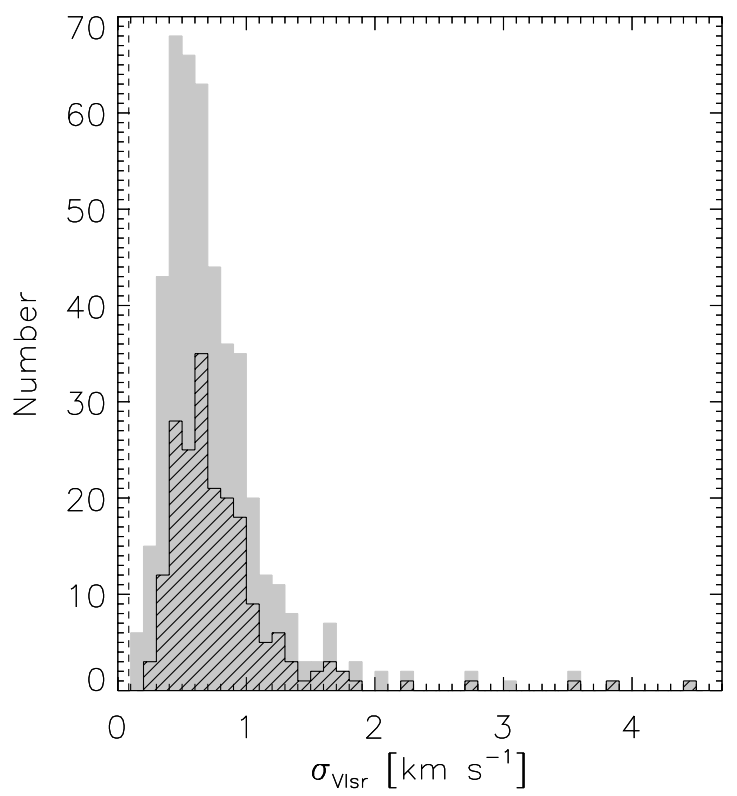

Figure 7. Distribution of Gaussian line widths for the full sample (gray histogram) and the $T_{\mathrm{K}}$ subsample (striped histogram). Note $\sigma_{\mathrm{Vlsr}}=$ FWHM $/ \sqrt{8 \ln 2}$. The dashed line denotes the resolution of the $\mathrm{NH}_{3}$ observations of $0.08 \mathrm{~km} \mathrm{~s}^{-1}$. Six of the eleven sources with $\sigma_{\mathrm{Vlsr}} \geqslant 2 \mathrm{~km} \mathrm{~s}^{-1}$ are weak detections, one has two velocity components that are poorly fit with the current model, and the remaining four sources truly do exhibit line widths greater than $2 \mathrm{~km} \mathrm{~s}^{-1}$.

We defer the analysis of the $\mathrm{H}_{2} \mathrm{O}$ maser observations to a later paper, but we do note the presence or absence of a water maser in Column 7 of Table 5. We have detected the $22 \mathrm{GHz} \mathrm{H}_{2} \mathrm{O}$ line toward 182 BGPS sources (40\%).

\section{RESOLVING THE KINEMATIC DISTANCE AMBIGUITY}

Without distances to the BGPS sources, the observed properties cannot be translated into physical properties. The observed $\mathrm{NH}_{3}$ radial velocity in conjunction with the Galactic rotation model of Reid et al. (2009; $R_{\mathrm{o}}=8.4 \pm 0.6 \mathrm{kpc}$, $\Theta_{0}=254 \pm 16 \mathrm{~km} \mathrm{~s}^{-1}$ ) provides kinematic distances to all 
sources with a detection in the $\mathrm{NH}_{3}(1,1)$ transition. However, the application of the Galactic rotation model introduces a distance ambiguity (known as the kinematic distance ambiguity or KDA) for sources within the solar circle. A single radial velocity provides two distances (referred to as a near and far distance) found on either side of the tangent point. Only sources found at the tangent velocity do not suffer from the KDA. We break the KDA based on the presence of infrared dark clouds (IRDCs) or H I self-absorption (HISA).

IRDCs are regions seen in absorption against the diffuse infrared background, some of which have been shown to have high densities $\left(>10^{5} \mathrm{~cm}^{-3}\right)$, cold temperatures $(<25 \mathrm{~K})$, and high column densities $\left(\sim 10^{23}-10^{25} \mathrm{~cm}^{-2}\right.$; Egan et al. 1998; Carey et al. 1998, 2000; Simon et al. 2006; Rathborne et al. 2005, 2006, 2007, 2008, 2010). Thousands have been cataloged from the MSX (Price et al. 2001) and the Galactic Legacy Infrared Mid-Plane Survey Extraordinaire (GLIMPSE; Benjamin et al. 2003; Chambers et al. 2009) surveys (Egan et al. 1998; Carey et al. 1998; Simon et al. 2006; Peretto \& Fuller 2009; Chambers et al. 2009). Spatial coincidence and structural similarity between a BGPS source and an IRDC place the BGPS source at the near kinematic distance where it is able to absorb the diffuse IR background. A BGPS source not coincident with an IRDC is assumed to be at the far kinematic distance. We have cross matched the BGPS sources observed in $\mathrm{NH}_{3}$ with the catalog of IRDCs seen in the Spitzer GLIMPSE (Peretto \& Fuller 2009) and identified 105 BGPS sources coincident with an IRDC. We have visually inspected the GLIMPSE $8 \mu \mathrm{m}$ images for spatial coincidence and structural similarity between the $1.1 \mathrm{~mm}$ emission and $8 \mu \mathrm{m}$ absorption features both identified by Peretto \& Fuller (2009) as well as those not included in their IRDC catalog. Forty-seven IRDCs from Peretto \& Fuller (2009) that were matched to a BGPS source were deemed not to be evidence of the near distance due to a mismatch in position or structure of the IRDC compared to the BGPS emission. Overall, 171 of the 456 BGPS sources in the full sample were found to coincide with an $8 \mu \mathrm{m}$ IRDC and were placed at the near kinematic distance, while 215 were assigned the far distance due to a lack of correspondence between $8 \mu \mathrm{m}$ absorption and $1.1 \mathrm{~mm}$ emission. Additionally, 70 BGPS sources had inconclusive evidence of an $8 \mu \mathrm{m}$ IRDC and were flagged as possibly at the near distance.

We note that the assumption that an IRDC is located at the near distance could be false in some cases. For example, since the identification of an IRDC relies on a bright infrared background, a source at the near distance without a significant infrared background to absorb would be falsely assigned to the far kinematic distance. Similarly, sources exhibiting weak $8 \mu \mathrm{m}$ absorption could be located at the far distance. Battersby et al. (2011) have identified a sample of five high column density sources in the Herschel infrared Galactic Plane Survey (Hi-GAL) data that have weak $8 \mu \mathrm{m}$ absorption and exhibit a discrepancy between the far-IR column density and that calculated from the $8 \mu \mathrm{m}$ absorption under the assumption of the near kinematic distance, which suggests the sources may be located at the far distance. While our assumption that a BGPS source associated with an IRDC is at the near distance is accurate in most cases, it disregards the fact that some IRDCs may be located at the far distance.

To minimize false kinematic distance assignments, we have also considered HISA when resolving the KDA. The HISA technique for resolving the KDA is similar to the IRDC method in that cold, dense material located at the near distance will absorb the emission from warmer material with the same radial velocity found at the far kinematic distance (e.g., Knapp 1974; Burton et al. 1978). The signature of HISA is frequently displayed in molecular clouds (e.g., Knapp 1974), and theoretical studies by Flynn et al. (2004) demonstrate that all molecular clouds contain enough $\mathrm{H}_{\mathrm{I}}$ to produce a self-absorption profile against a warm continuum background. Indeed, HISA has been seen throughout the Canadian Galactic Plane Survey of $21 \mathrm{~cm}$ emission toward the outer Galaxy (Gibson et al. 2000, 2005). This technique has recently been used to resolve the KDA toward star-forming regions and $\mathrm{H}$ II regions (Jackson et al. 2002; Fish et al. 2003; Busfield et al. 2006; Pandian et al. 2009; Anderson \& Bania 2009; Roman-Duval et al. 2010). There are situations in which this method for KDA resolution could be incorrect. For example, a BGPS source at the far distance could have a peculiar velocity that places it at the same velocity as a warmer source at the far distance and causes it to exhibit HISA. Also, an $\mathrm{H}$ II region embedded within a cold source at the far distance could also be incorrectly assigned to the near distance (RomanDuval et al. 2010). In such cases, the BGPS source would be incorrectly assigned to the near distance. Here we accept the KDA resolution as correct, but note that it may not be correct in all cases.

We have used the $21 \mathrm{~cm}$ Very Large Array Galactic Plane Survey (VGPS; Stil et al. 2006) and Southern Galactic Plane Survey (SGPS; McClure-Griffiths et al. 2005) to search for HISA toward the 456 BGPS sources in the full sample. Figure 8 shows examples of possible outcomes of the HISA method. We have marked the radial velocity observed in our $\mathrm{NH}_{3}$ observations, but use ${ }^{13} \mathrm{CO}(1-0)$ spectra from the BU-FCRAO GRS (Jackson et al. 2006) for comparison since ${ }^{13} \mathrm{CO}$ emission traces the line width and spatial extent of HISA well (e.g., Li \& Goldsmith 2003; Goldsmith \& Li 2005). The top panel shows a clear example of a HISA for BGPS source 2630. The green dashed line is the $\mathrm{H}_{\mathrm{I}}$ spectrum, the blue solid line is the ${ }^{13} \mathrm{CO}$ spectrum, and the dotted black line marks the observed $\mathrm{NH}_{3}$ velocity. The HISA feature matches both the observed $\mathrm{NH}_{3}$ velocity and the ${ }^{13} \mathrm{CO}$ peak and line width. The middle panel shows a spectrum toward a BGPS source with potential HISA. The H i spectrum has an absorption feature slightly offset from the observed $\mathrm{NH}_{3}$ velocity and ${ }^{13} \mathrm{CO}$ peak and the ${ }^{13} \mathrm{CO}$ line width does not match the potential absorption feature. The bottom panel shows a clear example a BGPS source placed at the far distance based on the HISA method. Here, the blue solid line displays the observed $\mathrm{NH}_{3}(1,1)$ spectrum since the GRS did not include our $7.5 \leqslant \ell \leqslant 10.0$ field. The $\mathrm{NH}_{3}$ hyperfine structure can clearly be seen in this spectrum, and the absorption features seen on either side of the $\mathrm{NH}_{3}$ emission are a result of the frequency switching performed during observations. The $\mathrm{H}$ I spectrum is smoothly decreasing at the $\mathrm{NH}_{3}$ velocity and shows no absorption feature. Of the 456 BGPS sources in the full sample, the HISA technique has identified 116 BGPS sources at the near distance, while 130 displayed questionable HISA and could not definitively break the KDA and 210 suggested the far distance due to a lack of HISA.

When investigating matches between a BGPS source and either an IRDC or HISA we assign one of three flags to each source for each method: " $y$ " denoting a positive association suggesting the near distance, " $n$ " denoting no association, and " $\mathrm{m}$ " representing a questionable association. A BGPS source is assigned the near distance if it receives a single " $y$ " (143 sources), two " $y$ " flags (72 sources), or two "m" flags, one from the IRDC and HISA methods (19 sources). Conversely, a BGPS 

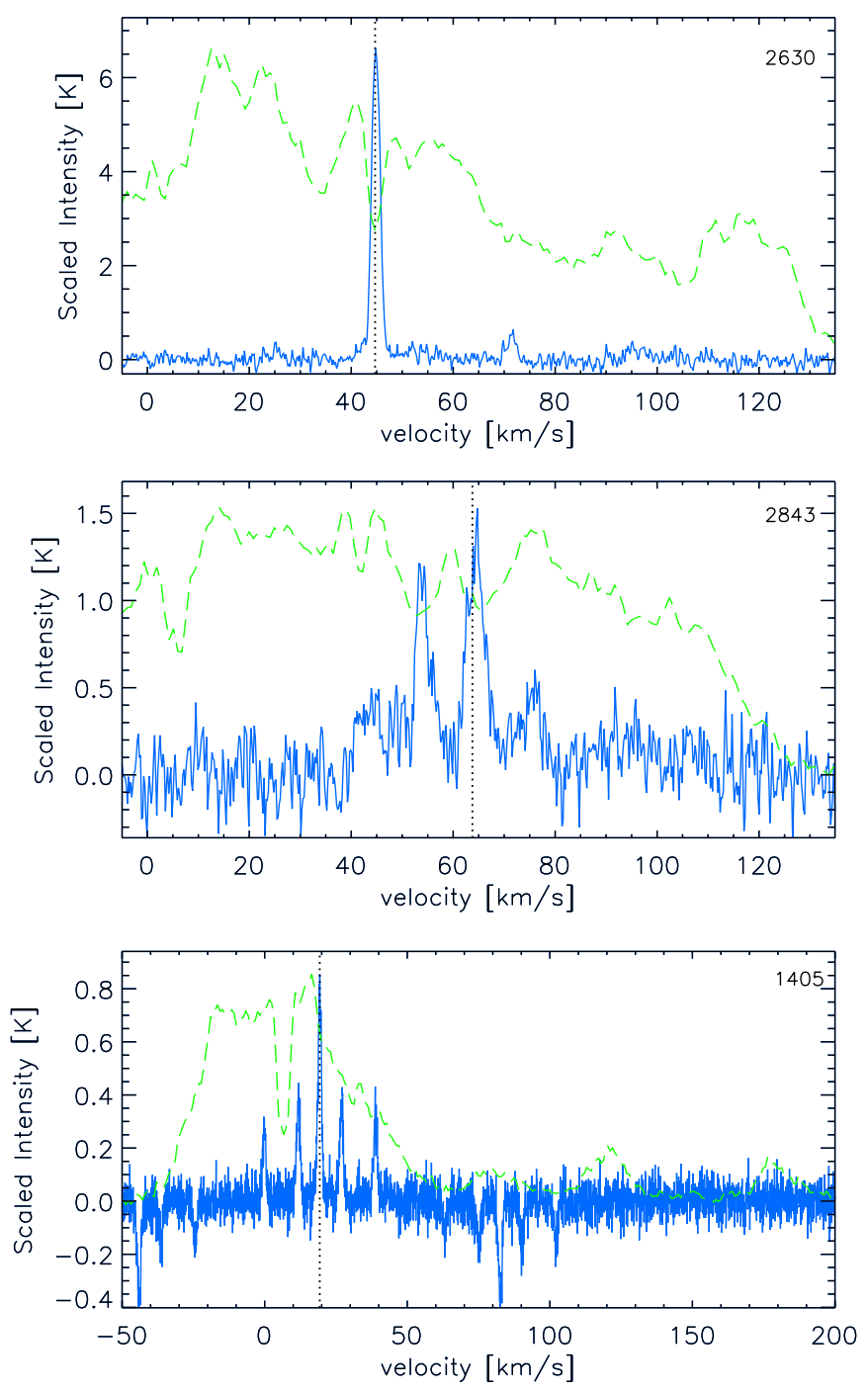

Figure 8. HISA examples. The green dashed line shows the Hi spectrum, the blue solid line shows the ${ }^{13} \mathrm{CO}$ spectrum (top and middle panels) or the observed $\mathrm{NH}_{3}(1,1)$ spectrum (bottom panel), and the dotted black line marks the observed $\mathrm{NH}_{3}$ velocity. Top panel: BGPS source 2630 . There is a very clear HISA feature at the same velocity as the ${ }^{13} \mathrm{CO}$ peak and the observed $\mathrm{NH}_{3}$ velocity. Middle panel: BGPS source 2843 with potential HISA. The width of the potential HISA feature and the offset in velocity from the dense gas tracers resulted in an " $m$ " flag for HISA. Bottom panel: BGPS source 1405 with no HISA. ${ }^{13} \mathrm{CO}$ observations were not available in the $7.5 \leqslant \ell \leqslant 10.0$ field; we overlay our $\mathrm{NH}_{3}$ spectrum instead. The two $\mathrm{NH}_{3}$ features seen in absorption on either side of the $\mathrm{NH}_{3}$ emission lines are due to the frequency switching used during observations. There is a clear lack of HISA at the same velocity as the $\mathrm{NH}_{3}$ spectrum.

(A color version of this figure is available in the online journal.)

source is assigned the far distance if it receives two "n" flags (126 sources) or one " $n$ " and one " $m$ " flag (96 sources).

By combining the IRDC and HISA results, we have identified 233 BGPS sources at the near distance, 221 at the far distance, and two at the tangent distance where the KDA does not exist. Table 6 lists the BGPS source ID (Column 1), HISA, IRDC, and KDA flags (Columns 2-4, respectively), as well as the assumed kinematic distance (Column 5; asymmetrical errors listed in parentheses), and resulting $R_{\text {Gal }}$ (Column 6). Figure 9 shows the distribution of distances for the full sample of 456 BGPS sources (gray histogram) and for the $T_{\mathrm{K}}$ subset (striped histogram). The peak in the distribution near $4 \mathrm{kpc}$ is likely a result of abundant dust and gas in the $5 \mathrm{kpc}$ molecular ring (e.g., Burton et al. 1975; Scoville \& Solomon 1975; Cohen \& Thaddeus 1977;

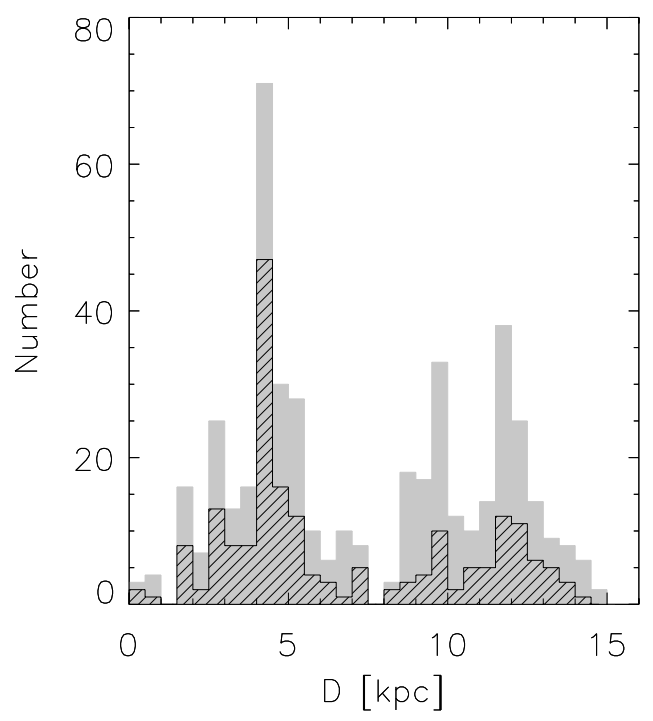

Figure 9. Distribution of kinematic distances. The gray histogram marks the full sample of 456 BGPS sources, while the striped histogram denotes the $T_{\mathrm{K}}$ subsample. The peak in the distribution around $4 \mathrm{kpc}$ is a result of the $5 \mathrm{kpc}$ molecular ring.

Table 6

Kinematic Distances

\begin{tabular}{lccccc}
\hline \hline $\begin{array}{l}\text { ID } \\
\text { Number }\end{array}$ & $\begin{array}{c}\text { IRDC } \\
\text { Flag }^{\mathrm{a}}\end{array}$ & $\begin{array}{c}\text { HISA } \\
\text { Flag }^{\mathrm{a}}\end{array}$ & $\begin{array}{c}\text { KDA } \\
\text { Flag }^{\mathrm{b}}\end{array}$ & $\begin{array}{c}\text { Kinematic } \\
\text { Distance }(\mathrm{kpc})\end{array}$ & $\begin{array}{c}R_{\text {Gal }} \\
(\mathrm{kpc})\end{array}$ \\
\hline 1307 & $\mathrm{n}$ & $\mathrm{n}$ & $\mathrm{f}$ & $12.4\left(_{-0.42}^{+0.52}\right)$ & 4.1 \\
1309 & $\mathrm{n}$ & $\mathrm{m}$ & $\mathrm{f}$ & $15.0\left(_{-1.00}^{+1.42}\right)$ & 6.6 \\
1310 & $\mathrm{~m}$ & $\mathrm{~m}$ & $\mathrm{n}$ & $6.9\left(_{-0.10}^{+0.10}\right)$ & 1.7 \\
1311 & $\mathrm{n}$ & $\mathrm{m}$ & $\mathrm{f}$ & $9.3\left(_{-0.09}^{+0.09}\right)$ & 1.4 \\
1313 & $\mathrm{y}$ & $\mathrm{y}$ & $\mathrm{n}$ & $0.2\left(_{-15.26}^{+2.45}\right)$ & 8.5 \\
1314 & $\mathrm{y}$ & $\mathrm{n}$ & $\mathrm{n}$ & $7.3\left(_{-0.09}^{+0.09}\right)$ & 1.4 \\
1316 & $\mathrm{y}$ & $\mathrm{m}$ & $\mathrm{n}$ & $7.3\left(_{-0.09}^{+0.09}\right)$ & 1.4 \\
1317 & $\mathrm{y}$ & $\mathrm{m}$ & $\mathrm{n}$ & $7.3\left(_{-0.09}^{+0.09}\right)$ & 1.4 \\
1320 & $\mathrm{n}$ & $\mathrm{n}$ & $\mathrm{f}$ & $13.7\left(_{-0.66}^{+0.87}\right)$ & 5.3 \\
1322 & $\mathrm{y}$ & $\mathrm{y}$ & $\mathrm{n}$ & $0.1\left(_{-1.44}^{+18.47}\right)$ & 8.2 \\
\hline
\end{tabular}

Notes. Errors are given in parentheses.

a $\mathrm{y}$ denotes positive association, $\mathrm{n}$ denotes no association, and $\mathrm{m}$ denotes questionable association.

${ }^{b} \mathrm{n}$ denotes near kinematic distance, $\mathrm{f}$ denotes far kinematic distance, and $\mathrm{t}$ denotes tangent distance.

(This table is available in its entirety in a machine-readable form in the online journal. A portion is shown here for guidance regarding its form and content.)

Robinson et al. 1984; Clemens et al. 1988; Kolpak et al. 2002; Rathborne et al. 2009). Figure 10 shows the location of the 456 BGPS sources in the Galaxy. The green crosses mark sources placed at the near distance, red squares mark sources at the tangent distance, while blue triangles mark sources placed at the far distance. The dotted line denotes the location of the tangent distance as a function of Galactic longitude.

As previously discussed, the use of IRDCs and HISA to resolve the KDA may lead to incorrect assignments of both near and far kinematic distances. To assess the possible effects of incorrect distance assignments, we have explored the worst case scenario, in which all sources have been assigned incorrect distances, by reversing the KDA resolution for all sources in the full sample. This test showed little effect on the statistical properties of the sample, suggesting that our results will not be greatly affected by incorrect distance assignments. 


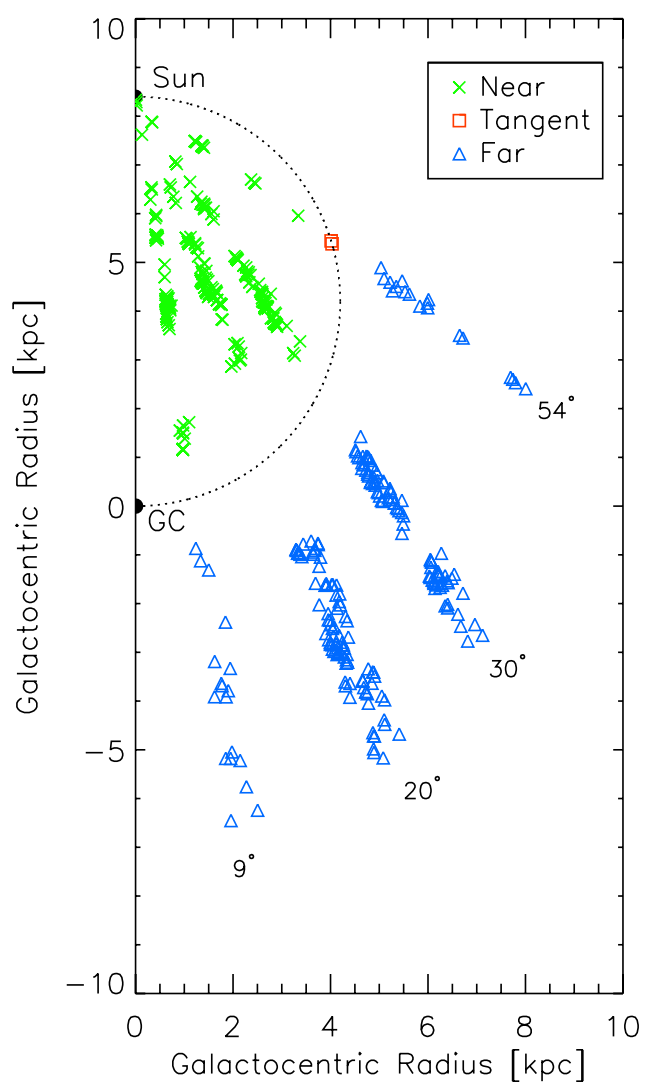

Figure 10. Location of the full sample of 456 BGPS sources in the Galaxy. Green crosses mark BGPS sources placed at the near kinematic distance, red squares mark sources at the tangent distance, and blue triangles mark sources placed at the far kinematic distance. The dotted line marks the location of the tangent distance as a function of Galactic longitude.

(A color version of this figure is available in the online journal.)

The use of a circular rotation curve for the Galaxy necessarily simplifies Galactic kinematics, and thus introduces errors into our assumed distances. For example, spiral arm structure can cause the radial velocities of nearby sources to deviate from circular rotation and display peculiar velocities. Similarly, the radial velocities of nearby sources that are orbiting the Galaxy at angular velocities similar to that of the Sun are dominated by peculiar velocities. Additionally, sources located within the Galactic bar exhibit streaming motions that can deviate significantly from circular rotation (e.g., Burton \& Bania 1974). In these cases, the observed radial velocities will return inaccurate kinematic distances under the assumption of simple circular rotation. Thus, kinematic distances have large inherent errors. Sources located in the $\ell \sim 8.5$ field could be strongly affected by the streaming motions within the bar. Sources in this region assigned small kinematic distances should be viewed skeptically. It is possible that peculiar velocities or streaming motions have resulted in a very low radial velocity and the source is truly located within the Galactic bar or Scutum spiral arm at approximately $4-5 \mathrm{kpc}$. These uncertainties should be noted; however, without a detailed model of the spiral arm structure, peculiar velocities and streaming motions throughout the Galaxy, we are unable to correct for these effects. In this paper, we accept the kinematic distances as correct but note the potential for large errors.

While the kinematic distance is subject to the near/far distance ambiguity in the inner Galaxy, each near/far distance pair corresponds to a single distance from the Galactic center,

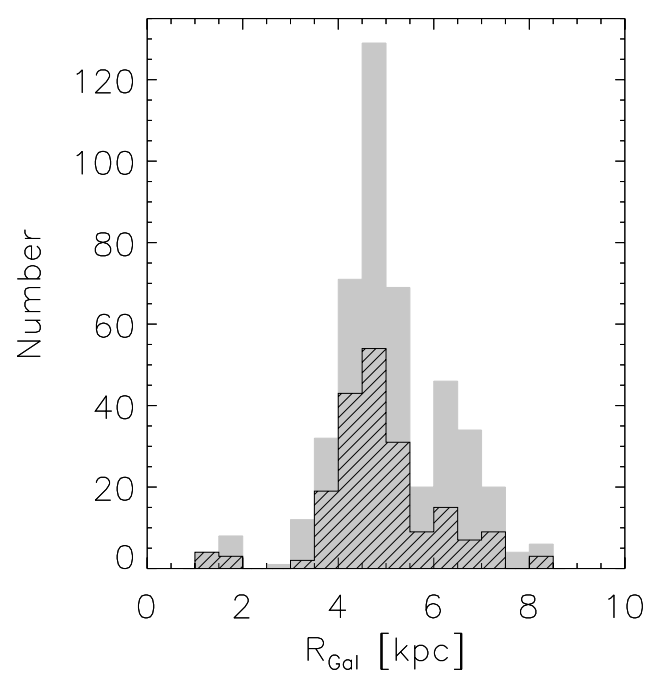

Figure 11. Distribution of Galactocentric radii for the full sample (gray histogram) and the $T_{\mathrm{K}}$ subsample (striped histogram). The peak in the number of sources at $R_{\mathrm{Gal}} \sim 4.5 \mathrm{kpc}$ corresponds to sources in the $5 \mathrm{kpc}$ molecular ring.

the Galactocentric radius. Figure 11 plots the distribution of Galactocentric radius for the full sample (gray histogram) and the $T_{\mathrm{K}}$ subsample (striped histogram). The peak in the distribution at $R_{\mathrm{Gal}}=4-5 \mathrm{kpc}$ corresponds to the $5 \mathrm{kpc}$ molecular ring, which corresponds to the end of the Galactic bar and beginning of the Scutum spiral arm. The peak in source number at $R_{\mathrm{Gal}}=6 \mathrm{kpc}$ corresponds to the Sagittarius arm. We do not have enough sources at large $R_{\text {Gal }}$ for a definitive detection or non-detection of the Perseus arm at $R_{\mathrm{Gal}}=8 \mathrm{kpc}$. Section 7 discusses trends in properties as a function of Galactocentric radius.

\section{ANALYSIS}

With kinematic distances, we are able to calculate physical properties such as mass and volume-averaged density. In this section, we present each property and describe the biases inherent in each. Errors are propagated through each derived quantity and are presented for individual sources within parentheses in Tables 1 and $4-7$.

When discussing mean properties here and throughout, the uncertainties given are the standard deviation about the mean rather than the uncertainty in the mean. Some property distributions are non-Gaussian, and the uncertainty given should be viewed as a measure of the scatter in the sample rather than the error.

General statistics (minimum, mean, standard deviation, median, and maximum) for the properties presented in this section for the full sample and the $T_{\mathrm{K}}$ subsample can be found in Table 8 .

\subsection{Physical Size}

We calculate the physical radius based on the object radius determined by the source extraction algorithm (Equation (1)) and the kinematic distance. The physical radii for the full sample are given in Column 2 of Table 7 and are shown in Figure 12(a). The gray circles are the $T_{\mathrm{K}}$ subsample and the gray crosses are BGPS sources with no $(2,2)$ detection. The black squares and the solid black line mark the mean physical radius within $2 \mathrm{kpc}$ bins. The short dashed line marks the physical radii corresponding to the beam radius of $16^{\prime \prime} .5$ as a function of distance, and the long dashed line marks the physical radii corresponding to 2'.95 (half of the spatial scale at which the BGPS loses sensitivity due 
Table 7

Derived Masses and Densities

\begin{tabular}{|c|c|c|c|c|c|c|c|c|c|c|c|}
\hline $\begin{array}{l}\text { ID } \\
\text { Number }\end{array}$ & $\begin{array}{l}\text { Radius } \\
\text { (pc) }\end{array}$ & $\begin{array}{c}M_{\mathrm{iso}}\left(120^{\prime \prime}\right) \\
\left(M_{\odot}\right)\end{array}$ & $\begin{array}{c}M_{\text {iso }}(\text { int }) \\
\left(M_{\odot}\right)\end{array}$ & $\begin{array}{c}M_{\mathrm{vir}}(\mathrm{int}) \\
\left(M_{\odot}\right)\end{array}$ & $\begin{array}{c}n_{p} \text { (int) } \\
\left(10^{3} \mathrm{~cm}^{-3}\right)\end{array}$ & $\begin{array}{c}n_{\mathrm{ex}}{ }^{\mathrm{a}} \\
\left(10^{3} \mathrm{~cm}^{-3}\right)\end{array}$ & $\begin{array}{c}\Sigma \text { (int) } \\
\left(10^{-2} \mathrm{~g} \mathrm{~cm}^{-2}\right)\end{array}$ & $\begin{array}{c}N_{\mathrm{NH}_{3}} \\
\left(10^{14} \mathrm{~cm}^{-2}\right)\end{array}$ & $\begin{array}{c}N_{\mathrm{H}_{2}}^{\mathrm{avg}} \\
\left(10^{21} \mathrm{~cm}^{-2}\right)\end{array}$ & $\begin{array}{c}N_{\mathrm{H}_{2}}^{\text {beam }} \\
\left(10^{22} \mathrm{~cm}^{-2}\right)\end{array}$ & $\begin{array}{c}X_{\mathrm{NH}_{3}} \\
\left(10^{-8}\right)\end{array}$ \\
\hline 1307 & 2.1 & 2621(690) & $2340(580)$ & $2169(100)$ & $0.99(0.33)$ & $\cdots$ & $3.44(1.01)$ & $10.07(0.93)$ & $7.35(2.15)$ & $1.19(0.18)$ & $13.69(4.20)$ \\
\hline 1309 & 2.1 & $1526(580)$ & $1632(590)$ & 2131(210) & $0.74(0.45)$ & $0.37(0.07)$ & $2.50(1.22)$ & $8.04(2.56)$ & $5.35(2.60)$ & $0.62(0.07)$ & $15.01(8.72)$ \\
\hline 1310 & 1.6 & $1163(240)$ & 985(230) & $665(28)$ & $0.97(0.24)$ & $0.76(0.15)$ & $2.54(0.62)$ & $6.47(0.76)$ & $5.43(1.32)$ & $1.00(0.23)$ & $11.93(3.23)$ \\
\hline 1311 & 1.2 & $952(150)$ & $904(120)$ & $879(11)$ & $2.33(0.34)$ & $1.33(0.27)$ & $4.42(0.64)$ & $18.41(0.50)$ & $9.46(1.33)$ & $0.81(0.09)$ & $19.45(2.78)$ \\
\hline 1313 & 0.0 & $0(100)$ & $0(34)$ & $10(490)$ & 230.61(81966.01) & $0.80(0.16)$ & $5.46(0.74)$ & $5.56(1.07)$ & $11.67(3278.20)$ & $0.90(0.21)$ & $4.76(1336.30)$ \\
\hline 1314 & 1.2 & 932(100) & 1013(90) & $1280(17)$ & $2.54(0.29)$ & $2.11(0.42)$ & $4.87(0.51)$ & $22.15(0.40)$ & $10.42(1.06)$ & $1.68(0.09)$ & $21.26(2.20)$ \\
\hline 1316 & 0.7 & $745(130)$ & 719(87) & 302(4) & $10.77(1.53)$ & $3.79(0.76)$ & $11.39(1.61)$ & $14.40(0.22)$ & $24.37(3.19)$ & $1.54(0.12)$ & $5.91(0.78)$ \\
\hline 1317 & 1.2 & $1238(190)$ & $947(150)$ & $1474(32)$ & $2.04(0.37)$ & $1.46(0.29)$ & $4.12(0.71)$ & $10.62(0.61)$ & $8.81(1.50)$ & $1.18(0.17)$ & $12.05(2.17)$ \\
\hline 1320 & 3.0 & $3019(980)$ & 2923(930) & $2002(140)$ & $0.45(0.21)$ & $1.13(0.23)$ & $2.20(0.86)$ & $4.53(0.91)$ & $4.71(1.84)$ & $0.82(0.18)$ & $9.61(4.22)$ \\
\hline 1322 & 0.0 & $0(63)$ & $0(56)$ & 1(240) & 799.73(929799.74) & $2.44(0.49)$ & $9.91(9102.62)$ & $12.08(0.22)$ & $21.19(19466.69)$ & $1.77(0.24)$ & $5.70(5235.07)$ \\
\hline
\end{tabular}

Notes. Errors are given in parentheses.

${ }^{a} n_{\text {ex }}$ cannot be calculated for sources where we have set $T_{\mathrm{K}}=T_{\mathrm{ex}}$.

(This table is available in its entirety in a machine-readable form in the online journal. A portion is shown here for guidance regarding its form and content.) 
Table 8

Statistical Summary

\begin{tabular}{|c|c|c|c|c|c|c|c|c|c|}
\hline \multirow[t]{2}{*}{ Property } & \multirow[t]{2}{*}{ Units } & \multicolumn{4}{|c|}{ Full Sample } & \multicolumn{4}{|c|}{$T_{\mathrm{K}}$ Subsample } \\
\hline & & Minimum & Mean $^{\mathrm{a}}$ & Median & Maximum & Minimum & Mean $^{\mathrm{a}}$ & Median & Maximum \\
\hline$D$ & $\mathrm{kpc}$ & 0.1 & $7.4(3.8)$ & 6.5 & 15.0 & 0.1 & $6.6(3.7)$ & 4.7 & 14.3 \\
\hline$R_{\mathrm{Gal}}$ & $\mathrm{kpc}$ & 1.4 & $5.1(1.2)$ & 4.8 & 8.5 & 1.4 & $4.9(1.2)$ & 4.7 & 8.2 \\
\hline$R_{\text {maj }}$ & $\mathrm{pc}$ & 0.01 & $1.20(0.77)$ & 1.04 & 4.79 & 0.01 & $1.28(0.77)$ & 0.93 & 5.65 \\
\hline$R_{\min }$ & $\mathrm{pc}$ & 0.00 & $0.79(0.49)$ & 0.68 & 2.75 & 0.01 & $0.84(0.49)$ & 0.67 & 2.88 \\
\hline$R$ & $\mathrm{pc}$ & 0.01 & $1.87(1.36)$ & 1.51 & 8.41 & 0.01 & $2.02(1.36)$ & 1.40 & 8.55 \\
\hline $\log \left(S_{v}\left(120^{\prime \prime}\right)\right)$ & $\mathrm{Jy}$ & -0.55 & $0.06(0.32)$ & 0.00 & 1.35 & -0.50 & $0.22(0.34)$ & 0.15 & 1.35 \\
\hline $\log \left(S_{v}\right.$ (int) $)$ & Jy & -0.88 & $0.06(0.40)$ & 0.01 & 1.48 & -0.54 & $0.24(0.41)$ & 0.16 & 1.48 \\
\hline$T_{\mathrm{mb}}(1,1)$ & $\mathrm{K}$ & 0.25 & $1.27(0.98)$ & 0.92 & 7.14 & 0.38 & $1.93(1.12)$ & 1.70 & 7.14 \\
\hline$T_{\mathrm{mb}}(2,2)$ & $\mathrm{K}$ & 0.17 & $0.59(0.54)$ & 0.41 & 4.73 & 0.24 & $0.91(0.68)$ & 0.73 & 4.73 \\
\hline$T_{\mathrm{ex}}(1,1)$ & $\mathrm{K}$ & 2.82 & $4.07(2.15)$ & 3.58 & 24.81 & 2.99 & $4.45(2.11)$ & 4.12 & 24.12 \\
\hline$T_{\mathrm{K}}$ & $\mathrm{K}$ & 5.00 & $15.57(5.02)$ & 14.24 & 59.41 & 11.95 & $17.39(5.49)$ & 15.70 & 59.41 \\
\hline$\left(T_{\mathrm{K}}-T_{\mathrm{ex}}\right)$ & K & 0.00 & $11.50(5.05)$ & 10.38 & 56.17 & 0.00 & $12.94(5.59)$ & 11.55 & 56.17 \\
\hline$V_{\mathrm{LSR}}$ & $\mathrm{km} \mathrm{s}^{-1}$ & -0.71 & $63.7(32.9)$ & 60.6 & 154.55 & 0.26 & $59.6(33.3)$ & 49.0 & 154.5 \\
\hline$\sigma_{v}$ & $\mathrm{~km} \mathrm{~s}^{-1}$ & 0.10 & $0.76(0.49)$ & 0.64 & 4.42 & 0.24 & $0.81(0.53)$ & 0.69 & 4.42 \\
\hline$a$ & $\mathrm{~km} \mathrm{~s}^{-1}$ & 0.13 & $0.23(0.03)$ & 0.22 & 0.46 & 0.20 & $0.24(0.03)$ & 0.23 & 0.46 \\
\hline$\sigma_{\mathrm{NT}}$ & $\mathrm{km} \mathrm{s}^{-1}$ & 0.08 & $0.75(0.49)$ & 0.63 & 4.41 & 0.23 & $0.81(0.53)$ & 0.68 & 4.41 \\
\hline $\operatorname{Mach}\left(\sigma_{\mathrm{Vlsr}} / a\right)$ & $\ldots$ & 0.59 & $3.23(1.87)$ & 2.83 & 19.38 & 1.12 & $3.24(1.73)$ & 2.85 & 15.47 \\
\hline$\tau(1,1)$ & $\ldots$ & 0.01 & $3.44(1.73)$ & 3.37 & 12.22 & 0.02 & 4.07(1.79) & 3.87 & 12.22 \\
\hline $\log \left(M_{\text {iso }}\left(120^{\prime \prime}\right)\right)$ & $M_{\odot}$ & -1.02 & $2.95(0.61)$ & 3.02 & 4.40 & -1.02 & $2.93(0.62)$ & 2.94 & 4.00 \\
\hline $\log \left(M_{\text {iso }}(\right.$ int $\left.)\right)$ & $M_{\odot}$ & -1.07 & $2.94(0.66)$ & 2.98 & 4.39 & -1.07 & $2.95(0.67)$ & 2.98 & 4.31 \\
\hline $\log \left(M_{\text {vir }}(\right.$ int $\left.)\right)$ & $M_{\odot}$ & -0.07 & $2.85(0.66)$ & 2.88 & 4.72 & 0.18 & $2.87(0.64)$ & 2.90 & 4.69 \\
\hline $\log \left(M_{\mathrm{vir}} / M_{\text {iso }}(\right.$ int $\left.)\right)$ & $\ldots$ & -1.38 & $-0.09(0.45)$ & -0.13 & 1.97 & -0.91 & $-0.08(0.42)$ & -0.13 & 1.97 \\
\hline $\log (n($ int $))$ & $\mathrm{cm}^{-3}$ & 2.07 & $3.12(0.56)$ & 3.03 & 5.90 & 2.15 & $3.27(0.58)$ & 3.22 & 5.90 \\
\hline$\Sigma($ int $)$ & $\mathrm{g} \mathrm{cm}^{-2}$ & 0.006 & $0.037(0.030)$ & 0.029 & 0.236 & 0.010 & $0.046(0.033)$ & 0.036 & 0.231 \\
\hline $\log \left(n_{\mathrm{ex}}\right)$ & $\mathrm{cm}^{-3}$ & 2.08 & $3.10(0.35)$ & 3.09 & 4.88 & 2.58 & $3.28(0.26)$ & 3.29 & 4.03 \\
\hline $\log \left(n_{\mathrm{ex}} / n\right.$ (int) $)$ & $\ldots$ & -2.51 & $-0.02(0.58)$ & 0.06 & 2.15 & -2.51 & $0.01(0.55)$ & 0.09 & 1.17 \\
\hline $\log \left(N_{\mathrm{NH}_{3}}\right)$ & $\mathrm{cm}^{-2}$ & 13.24 & $14.75(0.37)$ & 14.76 & 15.96 & 13.73 & $14.95(0.29)$ & 14.92 & 15.96 \\
\hline $\log \left(N_{\mathrm{H}_{2}}^{\mathrm{avg}}\right)$ & $\mathrm{cm}^{-2}$ & 21.08 & $21.81(0.27)$ & 21.79 & 22.70 & 21.32 & $21.91(0.27)$ & 21.89 & 22.69 \\
\hline $\log \left(N_{\mathrm{H}_{2}}^{\text {beam }}\right)$ & $\mathrm{cm}^{-2}$ & 21.30 & $22.09(0.27)$ & 22.06 & 23.29 & 21.55 & $22.22(0.28)$ & 22.18 & 23.29 \\
\hline $\log \left(N_{\mathrm{NH}_{3}} / N_{\mathrm{H}_{2}}^{\text {beam }}\right)$ & $\ldots$ & -8.63 & $-7.34(0.30)$ & -7.33 & -6.34 & -8.36 & $-7.27(0.26)$ & -7.27 & -6.56 \\
\hline
\end{tabular}

Note. ${ }^{\text {a }}$ Standard deviation is given in parentheses.

to spatial filtering). BGPS sources with extracted radii smaller than half the beam size have been set as upper limits with radii equal to half the beam size. The mean physical radius increases with distance as expected due to the upper limit on spatial scales imposed by spatial filtering during data reduction and the lower limit due to the beam size.

Typical radii limits for cores, clumps, and clouds are denoted with the dotted lines at $0.125 \mathrm{pc}$ and $1.25 \mathrm{pc}$ (cores $R=$ $0.01-0.1 \mathrm{pc}$, clumps $R=0.15-1.5 \mathrm{pc}$, clouds $R=2.0-7.5 \mathrm{pc}$; Bergin \& Tafalla 2007) in Figure 12. Assuming that BGPS sources have a constant density, we can place constraints on the distances at which each type of source would be included in the BGPS catalog. Cores farther than $1.25 \mathrm{kpc}$ will be unresolved by the $33^{\prime \prime}$ beam (for $R=0.1 \mathrm{pc}$ ), although unresolved cores can still be detected by the BGPS if they have flux densities high enough to counterbalance beam-averaging effects and remain above the beam-averaged detection threshold (see Section 7.2 of D10). The largest $(R=1.5 \mathrm{pc})$ uniform density clumps at distances less than $1.75 \mathrm{kpc}$ would be filtered out but would be resolved across the Galaxy. The smallest clumps $(R=0.15 \mathrm{pc})$ would become unresolved relative to the beam at $1.9 \mathrm{kpc}$. Similarly, the smallest clouds would be filtered out at distances closer than $1.2 \mathrm{kpc}$, while the largest would be filtered out if closer than $8.7 \mathrm{kpc}$. The largest clouds are resolved even at the far side of the Galaxy.

The mean $1.1 \mathrm{~mm}$ source radius increases with distance and sources are extended compared to the $33^{\prime \prime}$ beam. There are
24 unresolved BGPS sources for which we will also need to consider mass in order to place them in the hierarchical structure.

\subsection{Gas Kinetic Temperature}

The gas kinetic temperature is calculated based on the excitation temperatures of the observed $\mathrm{NH}_{3}$ lines. For sources with only the $(1,1)$ line detected, the rms of the $(2,2)$ spectra was used as an upper limit to calculate the $(2,2)$ excitation temperature and the resulting $T_{\mathrm{K}}$ is only an upper limit. The uncertainties in individual $T_{\mathrm{K}}$ measurements are small, with $\left\langle\sigma_{T_{\mathrm{K}}} / T_{\mathrm{K}}\right\rangle=0.06$.

The derived $T_{\mathrm{K}}$ are given in Column 3 of Table 5. The full sample is characterized by $\left\langle T_{\mathrm{K}}\right\rangle<15.6 \pm 5.0 \mathrm{~K}$, while the $T_{\mathrm{K}}$ subsample is characterized by $\left\langle T_{\mathrm{K}}\right\rangle=17.4 \pm 5.5 \mathrm{~K}$. The fractional scatter about the mean $(0.32)$ is five times larger than the individual uncertainties in the measurements (0.06) so the differences among the sources are real. See Table 8 for further statistics. There are 81 sources that likely require multiple $T_{\mathrm{K}}$ components to fit the $\mathrm{NH}_{3}(3,3)$ emission as well as the $(1,1)$ and $(2,2)$. These sources are marked in Column 8 of Table 5.

Without mapping the $\mathrm{NH}_{3}$ emission toward the BGPS sources we cannot characterize kinetic temperature gradients in the gas. However, since the $\mathrm{NH}_{3}$ pointings correspond to the peak $1.1 \mathrm{~mm}$ emission, which closely traces the $\mathrm{NH}_{3}$ emission (Friesen et al. 2009), the measured $T_{\mathrm{K}}$ is likely an upper limit as $T_{\mathrm{K}}$ has been shown to increase toward the peak of the $\mathrm{NH}_{3}$ 


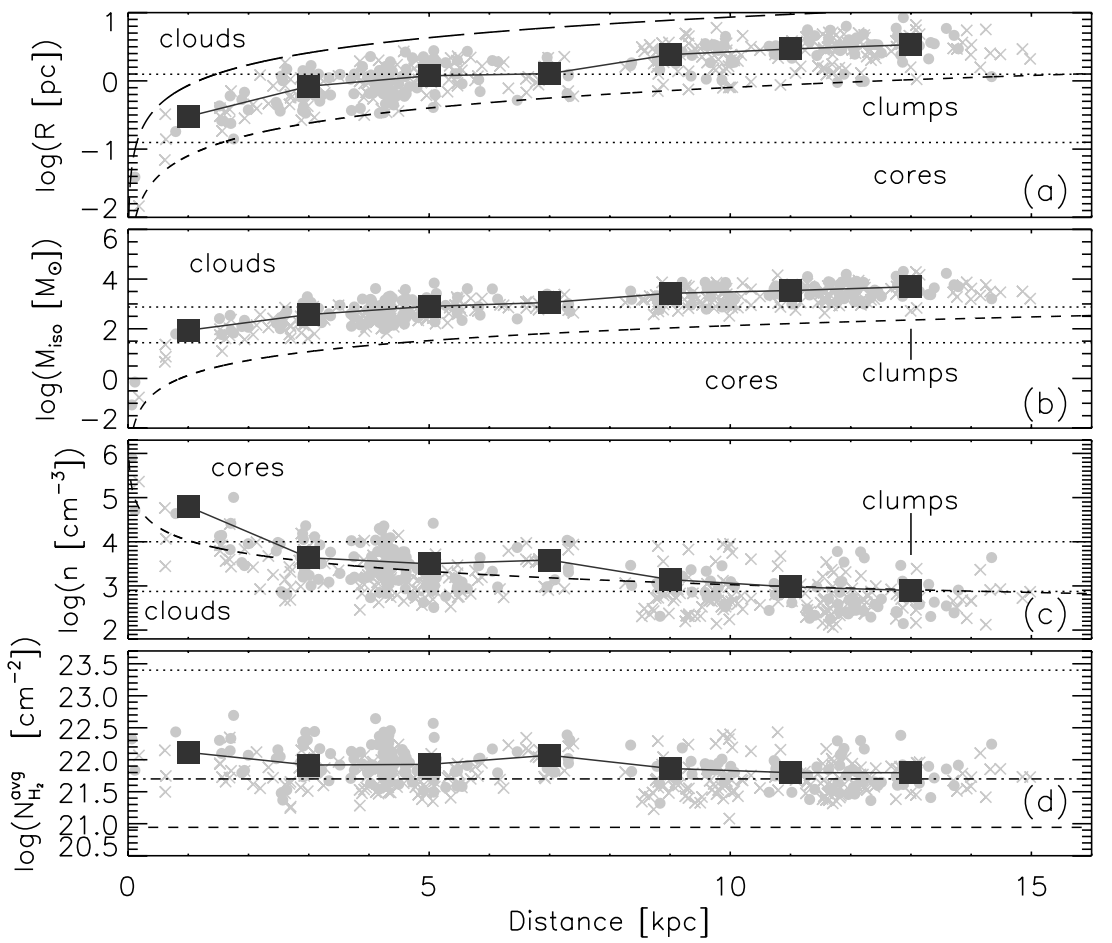

Figure 12. Physical properties vs. kinematic distance. In all panels, the dotted lines delineate typical properties of clouds, clumps, and cores while the short dashed lines mark lower limits of the physical properties within a $33^{\prime \prime}$ beam. The gray circles mark the $T_{\mathrm{K}}$ subsample and gray crosses mark sources with no $\mathrm{NH}_{3}(2,2)$ detection. The black squares and lines denote the mean of each property within $2 \mathrm{kpc}$ wide bins centered at 1, 3, 5, 7, 9, 11, and 13 kpc. (a) Physical radius. The long dashed line denotes the upper limit to source size imposed by the spatial filtering of uniform emission on size scales greater than 5'.9. (b) $M_{\text {iso }}$ (int). (c) Volume-averaged density. (d) $\mathrm{H}_{2}$ column density. The dotted line in panel (d) marks the surface density of $1 \mathrm{~g} \mathrm{~cm}^{-2}$ required to prevent fragmentation and allow a massive star to form (Krumholz \& McKee 2008). The dash-dot line denotes the threshold gas surface density required for efficient star formation as seen in nearby low-mass star-forming regions (Lada et al. 2010; Heiderman et al. 2010).

emission (Zinchenko et al. 1997). Thus, the $\mathrm{NH}_{3}$ outside of the GBT beam is likely to be colder than the $T_{\mathrm{K}}$ observed toward the peak of the $1.1 \mathrm{~mm}$ emission.

\subsection{Isothermal and Virial Masses}

Assuming that the dust can be characterized by a single temperature, we calculate the isothermal mass, $M_{\text {iso }}$, as

$$
\begin{aligned}
M_{\text {iso }}= & \frac{S_{v} D^{2}}{\kappa_{\nu} B_{v}\left(T_{\mathrm{D}}\right)}=13.1 M_{\odot}\left(\frac{S_{v}}{1 \mathrm{Jy}}\right)\left(\frac{D}{1 \mathrm{kpc}}\right)^{2} \\
& \times\left(\frac{e^{13.0 \mathrm{~K} / T_{\mathrm{D}}}-1}{e^{13.0 \mathrm{~K} / 20.0 \mathrm{~K}}-1}\right),
\end{aligned}
$$

where $S_{v}$ is the $1.1 \mathrm{~mm}$ flux density, $D$ is the kinematic distance, $\kappa_{\nu}$ is the dust opacity per gram of dust and gas and includes a gas-to-dust ratio of 100, and $B_{v}$ is the Planck function evaluated at $T_{\mathrm{D}}$. We logarithmically interpolate the Ossenkopf \& Henning (1994) dust opacities (Table 1, Column 5, commonly referred to as $\mathrm{OH} 5$ dust) to $271.1 \mathrm{GHz}$ (the effective central frequency of the Bolocam bandpass convolved with a $20 \mathrm{~K}$ blackbody modified by an opacity varying with frequency as $\kappa_{v} \propto v^{1.8}$; see Aguirre et al. 2011) and find $\kappa_{v}=0.0114 \mathrm{~cm}^{2} \mathrm{~g}^{-1}$.

We make the simplifying assumption that the dust and gas are collisionally coupled such that $T_{\mathrm{D}}=T_{\mathrm{K}}$ and use the derived $T_{\mathrm{K}}$ in calculating $M_{\mathrm{iso}}$. As noted in Section 6.2, the derived $T_{\mathrm{K}}$ are likely an upper limit to the average $T_{\mathrm{K}}$ for an entire BGPS source. Thus, the assumption that $T_{\mathrm{D}}=T_{\mathrm{K}}$ characterizes the entire BGPS source will overestimate $T_{\mathrm{D}}$ and underestimate $M_{\text {iso }}$. As discussed in D10, based on $\mathrm{NH}_{3}$ maps of molecular clouds presented in Zinchenko et al. (1997), assuming the peak
$T_{\mathrm{K}}$ for the entire source may underestimate $M_{\text {iso }}$ by a factor of up to two. Additionally, upper limits due to non-detections of the $\mathrm{NH}_{3}(2,2)$ line provide only lower limits on $M_{\text {iso }}$.

$M_{\text {iso }}$ could also be overestimated if some of the $1.1 \mathrm{~mm}$ emission is radio free-free emission rather than thermal dust emission. Dunham et al. (2011) found that the mean contribution of free-free emission is only $3 \%$ based on a comparison of BGPS flux densities and $6 \mathrm{~cm}$ radio emission from Urquhart et al. (2009). For most BGPS sources, free-free emission will not significantly increase the flux density, but for a small number of sources approximately half or more of the $1.1 \mathrm{~mm}$ flux density could be a result of free-free emission.

$M_{\text {iso }}\left(120^{\prime \prime}\right)$ and $M_{\text {iso }}$ (int) are listed in Table 7 . The distributions of $M_{\text {iso }}\left(120^{\prime \prime}\right)$ and $M_{\text {iso }}$ (int) are shown in Figure 13 for the full sample (gray histograms) and the $T_{\mathrm{K}}$ subsample (striped histograms). The $T_{\mathrm{K}}$ subsample is characterized by $\left\langle\log \left(M_{\text {iso }}\left(120^{\prime \prime}\right) / M_{\odot}\right)\right\rangle=2.95 \pm 0.61$ and $\left\langle\log \left(M_{\text {iso }}(\right.\right.$ int $\left.\left.) / M_{\odot}\right)\right\rangle=2.94 \pm 0.66$. See Table 8 for additional statistics for these mass distributions. The lowest mass sources $\left(M_{\text {iso }}<10 M_{\odot}\right)$ at distances less than $1 \mathrm{kpc}$ and with velocities near $0 \mathrm{~km} \mathrm{~s}^{-1}$ have unreliable kinematic distances as discussed in Section 5.

We also calculate the virial mass for each source assuming a spherical, uniform density gas cloud given by

$$
M_{\mathrm{vir}}=\frac{5 \sigma_{\mathrm{Vlsr}}^{2} R}{G},
$$

where $\sigma_{\mathrm{Vlsr}}$ is the Gaussian line width, $R$ is the physical radius, and $G$ is the gravitational constant. This equation assumes that the $\mathrm{NH}_{3}$ line width observed at the peak of the $1.1 \mathrm{~mm}$ emission is also valid at the edge of the observed $1.1 \mathrm{~mm}$ emission. This 


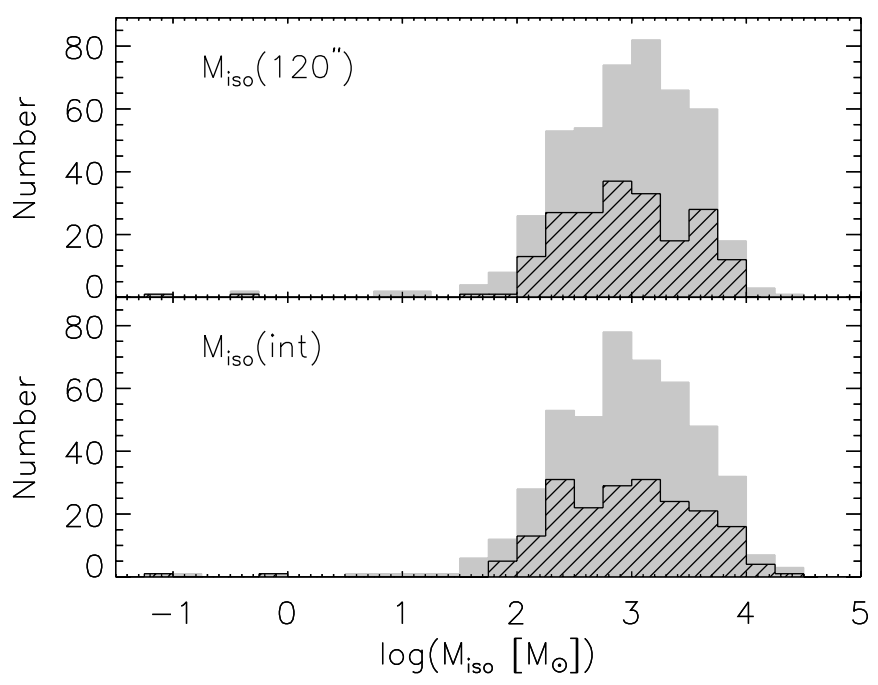

Figure 13. Distribution of isothermal masses $M_{\mathrm{iso}}\left(120^{\prime \prime}\right)$ (top panel) and $M_{\text {iso }}$ (int) (bottom panel) for the full sample (gray histograms) and the $T_{\mathrm{K}}$ subsample (striped histograms).

assumption is unlikely to hold for the larger BGPS sources at the farthest distances, as $\mathrm{NH}_{3}$ emission is not likely to trace entire molecular clouds but rather the denser regions within the clouds. As discussed in D10, these assumptions likely result in an overestimate of $M_{\text {vir }}$. Zinchenko et al. (1997) found that the line width decreases away from the peak of the $\mathrm{NH}_{3}$ emission in approximately half of their sample, which would lead to an overestimate of $M_{\mathrm{vir}}$. Additionally, the BGPS sources are not likely spherical or uniform density. The mean aspect ratio of the full sample is $1.5 \pm 0.3$. We cannot characterize the density distribution of the BGPS sources with our current data set, so we consider the mean power-law density profile found from modeling dust emission from massive star-forming regions, $\langle p\rangle=1.8 \pm 0.4$ (Mueller et al. 2002). If we correct for shape and non-uniform density as described in D10, $\left\langle M_{\mathrm{vir}} / M_{\text {iso }}\right\rangle$ would be decreased by $36 \%$. Since we do not have a measure of the density power law, or $\mathrm{NH}_{3}$ maps to quantify these effects for each BGPS source, we do not include these corrections in subsequent analysis but note that the virial masses presented are upper limits.

$M_{\text {vir }}$ (int) is given in Table 7 . The full sample is characterized by $\left\langle\log \left(M_{\mathrm{vir}} / M_{\odot}\right)\right\rangle=2.85 \pm 0.66$ while the $T_{\mathrm{K}}$ subsample is characterized by $\left\langle\log \left(M_{\text {vir }} / M_{\odot}\right)\right\rangle=2.87 \pm 0.64$. Figure 14 plots the virial parameter, $M_{\mathrm{vir}} / M_{\mathrm{iso}}$, versus $M_{\mathrm{iso}}$ (int). The full sample is characterized by $\left\langle\log \left(M_{\text {vir }} / M_{\text {iso }}\right)\right\rangle=-0.09 \pm 0.45$ and a median of $10^{-0.13}$, which is more representative of the full sample since the mean is skewed by the few sources with a virial parameter of $\sim 100$. These BGPS sources with $M_{\text {vir }} / M_{\text {iso }} \sim 100$ are the local sources with unreliable kinematic distances and upper limits for the source sizes. Since the virial parameter is proportional to $D^{-1}$, the observed scatter in the virial parameter could be a result of errors in the derived kinematic distances. Additionally, the scatter could be due to deviations from a uniform density distribution or varying source ellipticities. Alternatively, the assumption that the observed line width is applicable at the boundary of the $1.1 \mathrm{~mm}$ emission could be invalid.

$M_{\text {iso }}$ (int) is shown versus kinematic distance in Figure 12(b). The gray circles mark the $T_{\mathrm{K}}$ subsample, and the gray crosses mark BGPS sources with an upper limit for $T_{\mathrm{K}}$, and subsequently a lower limit in $M_{\text {iso }}$. The dashed black line marks the mass

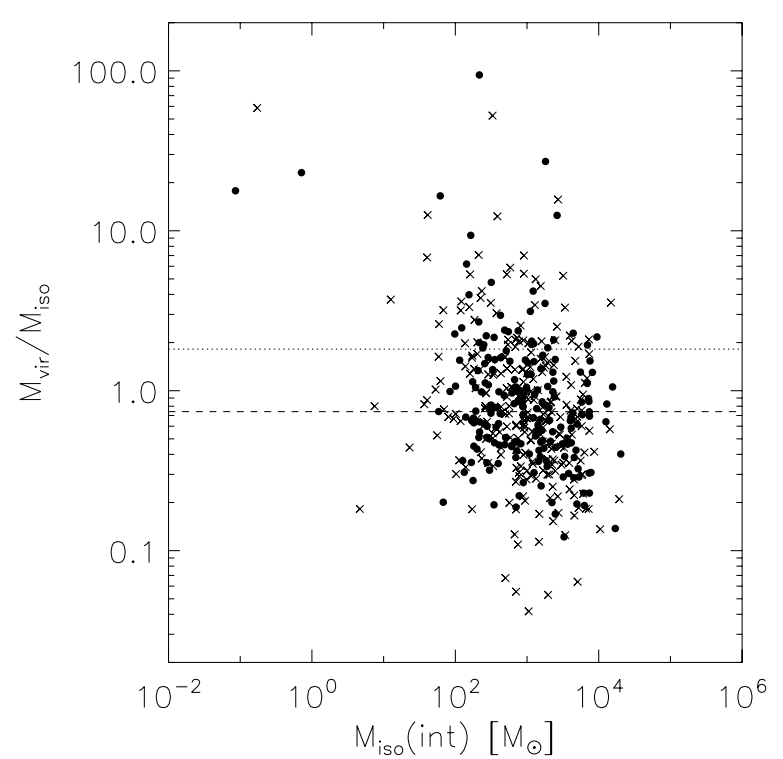

Figure 14. $M_{\mathrm{vir}} / M_{\mathrm{iso}}$ (int) ratio vs. $M_{\mathrm{iso}}$ (int) for the full sample. The dotted line marks the mean virial parameter of the full sample, $\left\langle M_{\mathrm{vir}} / M_{\mathrm{iso}}\right\rangle=1.82$, and the dashed line marks the median virial parameter of 0.74 .

within a beam corresponding to a $5 \sigma$ detection, where we assume $\sigma=0.020 \pm 0.006 \mathrm{Jy}^{\text {beam }^{-1}}$ from the mean and standard deviation of the rms in each BGPS field, and $T_{\mathrm{D}}=$ $20 \mathrm{~K}$. The black squares and black line connecting them mark the mean $M_{\text {iso }}$ within $2 \mathrm{kpc}$ wide bins. The dotted lines mark limits between clouds, clumps, and cores of $27.5 M_{\odot}$ and $750 M_{\odot}$ based on the following characteristic values of each type: clouds have $M=10^{3}-10^{4} M_{\odot}$, clumps have $M=50-500 M_{\odot}$, and cores have $M=0.5-5 M_{\odot}$ (Bergin \& Tafalla 2007). The mean $M_{\text {iso }}$ increases with distance, and all masses are above the limiting mass per beam because the sources are significantly extended compared to the beam.

\subsection{Sound Speed and Non-thermal Motions}

The observed line widths provide a measure of the internal motions within each BGPS source. As discussed in Section 4, we observe velocity dispersions of up to $4 \mathrm{~km} \mathrm{~s}^{-1}$, with $98 \%$ of sources having $\sigma_{\mathrm{Vlsr}} \leqslant 2.0 \mathrm{~km} \mathrm{~s}^{-1}$, which is similar to line widths observed toward other samples of massive starforming regions (e.g., $\Delta V(\mathrm{FWHM}) \sim 1-4 \mathrm{~km} \mathrm{~s}^{-1}$; Anglada et al. 1996) but an order of magnitude larger than seen in $\mathrm{NH}_{3}$ observations of cores in nearby, low-mass star-forming regions (e.g., Rosolowsky et al. 2008).

The observed velocity dispersion is a result of both thermal and non-thermal motions of the gas. Given the derived $T_{\mathrm{K}}$ we can calculate the thermal contribution to the line width and remove it to determine the non-thermal velocity dispersion:

$$
\sigma_{\mathrm{NT}}=\sqrt{\sigma_{\mathrm{Vlsr}}^{2}-\frac{k T_{\mathrm{K}}}{17 m_{\mathrm{H}}}},
$$

where $\left(k T_{\mathrm{K}} / 17 m_{\mathrm{H}}\right)^{1 / 2}$ is the thermal broadening due to $\mathrm{NH}_{3}$, and $m_{\mathrm{H}}$ is the mass of a single hydrogen atom. The full sample can be characterized by $\left\langle\sigma_{\mathrm{NT}}\right\rangle<0.75 \pm 0.49 \mathrm{~km} \mathrm{~s}^{-1}$, and the $T_{\mathrm{K}}$ subsample by $\left\langle\sigma_{\mathrm{NT}}\right\rangle=0.81 \pm 0.53 \mathrm{~km} \mathrm{~s}^{-1}$. The non-thermal velocity dispersions for the full sample are shown in Figure 15 versus $T_{\mathrm{K}}$. The $T_{\mathrm{K}}$ subsample is shown as black circles, while sources without a $(2,2)$ detection and an upper limit in $T_{\mathrm{K}}$ are plotted as arrows marking the upper-limit in $T_{\mathrm{K}}$. The solid black 

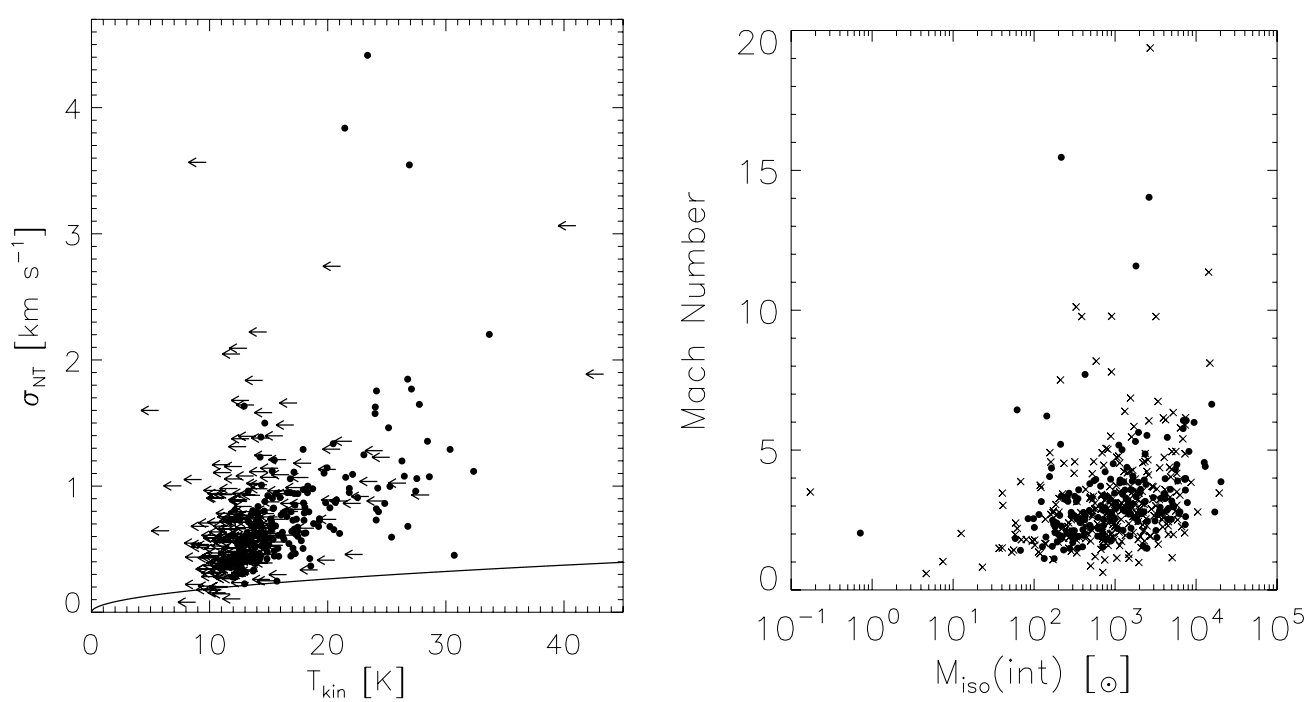

Figure 15. Left: the non-thermal line width vs. $T_{\mathrm{K}}$ for the full sample. Black circles mark the $T_{\mathrm{K}}$ subsample and the arrows mark sources with only an upper limit for $T_{\mathrm{K}}$ due to a non-detection in the $(2,2)$ line. The black line denotes the thermal sound speed given by $a=\left(k T_{\mathrm{K}} / \mu m_{\mathrm{H}}\right)^{1 / 2}$. The non-thermal component is, on average, three times larger than the thermal sound speed. Right: mach number $\left(\sigma_{\mathrm{Vlsr}} / \sqrt{k T_{\mathrm{K}} / \mu m}\right)$ vs. $M_{\text {iso }}($ int $)$. Here, crosses mark sources without a $(2,2)$ detection.

line depicts the thermal sound speed as a function of $T_{\mathrm{K}}$ given by $a=\left(k T_{\mathrm{K}} / \mu m_{\mathrm{H}}\right)^{1 / 2}$, where $\mu=2.37$. For most sources, the non-thermal line width is significantly larger than the thermal component; we find a mean ratio of non-thermal to thermal sound speed of $3.2 \pm 1.8$ for the full sample and $3.2 \pm 1.7$ for the $T_{\mathrm{K}}$ subsample. The sound speed and non-thermal velocity dispersion are listed in Columns 5 and 6 of Table 5.

The left panel of Figure 15 plots the Mach number (given as $\sigma_{\mathrm{Vlsr}} / a$ ) versus $M_{\text {iso }}($ int). The non-thermal velocity dispersion weakly scales with mass. As discussed in the previous section, $M_{\text {iso }}$ increases with distance, and the increase in $\sigma_{\mathrm{NT}}$ seen with mass is likely the result of observing larger objects at greater distances. We find a mean Mach number of $3.23 \pm 1.87$ for the full sample and $3.24 \pm 1.73$ for the $T_{\mathrm{K}}$ subsample.

\subsection{Volume and Surface Densities}

We calculate the volume-averaged density based on $M_{\text {iso }}$ (int) and $R$ as $n=3 M_{\text {iso }}$ (int) $/ 4 \pi \mathrm{R}^{3}$. This quantity is listed in Column 6 of Table 7, and shown in Figure 12(c). The dashed line marks the volume-averaged density corresponding to the limiting mass within a beam size shown in panel (b) and described in Section 6.3. In contrast to the limiting mass, this measure of density is not a hard limit. It is possible to have lower and higher density BGPS sources. For example, a BGPS source with an extracted size equal to the beam size will have a higher density if the flux is greater than the $5 \sigma$ limit required for detection. A large, extended BGPS source with an extracted size much larger than the beam size can have a density lower than this limit. The observed flux is roughly linear with source size while the volume is proportional to $R^{3}$, thereby allowing a larger source to have a lower density (see Section 7.2 of D10). This trend can be seen in Figure 12(c) where the volumeaveraged density decreases with kinematic distance while the extracted $1.1 \mathrm{~mm}$ source size increases. The dotted lines in this figure denote the typical range of clouds, clumps, and cores (clouds, $n=50-500 \mathrm{~cm}^{-3}$; clumps, $n=10^{3}-10^{4} \mathrm{~cm}^{-3}$; cores, $n=10^{4}-10^{5} \mathrm{~cm}^{-3}$; Bergin \& Tafalla 2007).

The full sample has a minimum volume-averaged density of $120 \mathrm{~cm}^{-3}$, a maximum of $8.0 \times 10^{5} \mathrm{~cm}^{-3}$, and $\left\langle\log \left(n / \mathrm{cm}^{-3}\right)\right\rangle=$ $3.12 \pm 0.56$. The $T_{\mathrm{K}}$ subsample has $\left\langle\log \left(n / \mathrm{cm}^{-3}\right)\right\rangle=3.27 \pm$

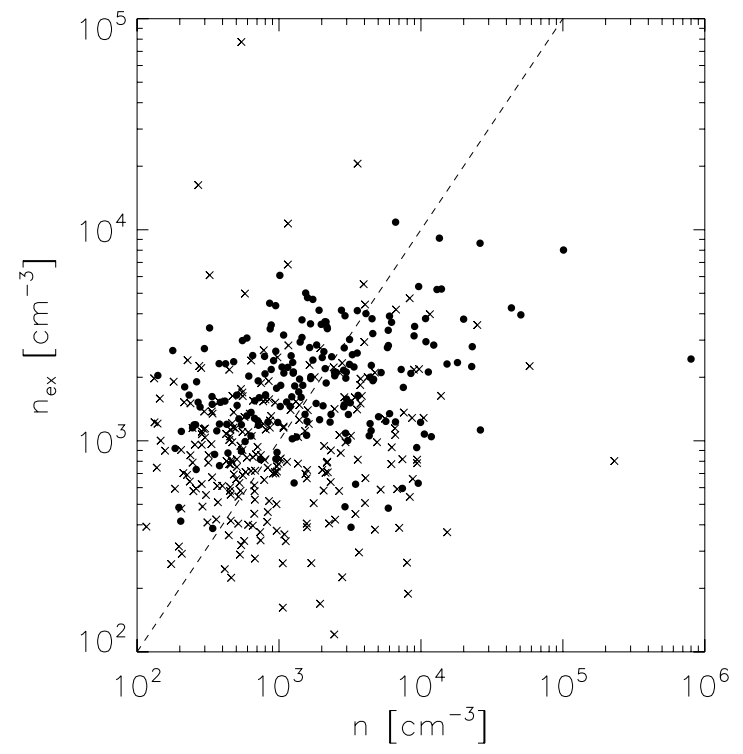

Figure 16. Observed $T_{\mathrm{mb}}(1,1)$ vs. kinematic distance for the full sample. Circles mark the $T_{\mathrm{K}}$ subsample and crosses mark sources without a $(2,2)$ detection. The decrease in observed $T_{\mathrm{mb}}(1,1)$ with distance is indicative of beam dilution.

0.58. Similarly, we calculate the average surface density as $\Sigma=M_{\text {iso }}($ int $) / \pi R^{2}$. This quantity is listed in Column 8 of Table 7. The distribution of surface density for the full sample ranges from $0.006 \mathrm{~g} \mathrm{~cm}^{-2}$ to $0.24 \mathrm{~g} \mathrm{~cm}^{-2}$ with $\langle\Sigma\rangle=$ $0.037 \pm 0.030 \mathrm{~g} \mathrm{~cm}^{-2}$. The full statistical characteristics of each sample are given in Table 8.

\subsection{Excitation Temperature and Density}

The observed $T_{\mathrm{mb}}(1,1)$ are shown versus kinematic distance in Figure 16, where circles mark the $T_{\mathrm{K}}$ subsample and crosses mark sources without a $(2,2)$ detection. There is a clear decrease in $T_{\mathrm{mb}}(1,1)$ with distance suggesting that distant sources suffer from beam dilution. Such beam dilution causes $T_{\mathrm{ex}}$ to be underestimated, which in turn underestimates the excitation density as discussed below. Such beam dilution will not affect the calculated $T_{\mathrm{K}}$ since it is based on the ratio of $(1,1)$ and $(2,2)$ emission, which will suffer from the same beam dilution. 


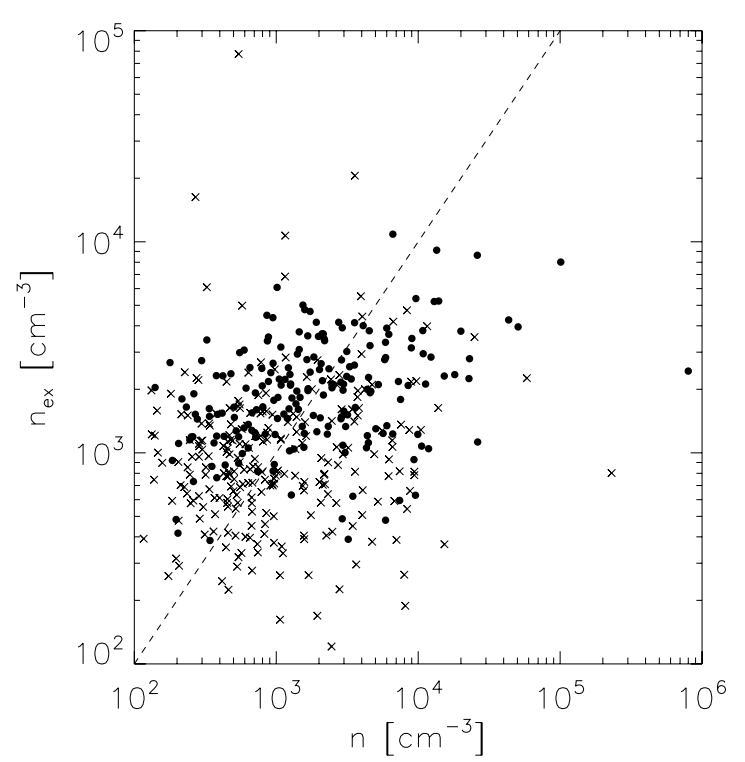

Figure 17. Excitation density vs. $1.1 \mathrm{~mm}$ volume-averaged density. Black circles mark the $T_{\mathrm{K}}$ subsample while crosses mark sources with only an upper limit for $T_{\mathrm{K}}$. The dashed line denotes a one-to-one correlation and is not a fit to the data.

The measured excitation temperatures are listed in Table 5. Sources not well fit by the assumed $\mathrm{NH}_{3}$ model have arbitrarily been set to have $T_{\mathrm{ex}}=T_{\mathrm{K}}$. The full sample is characterized by $\left\langle T_{\text {ex }}\right\rangle=4.1 \pm 2.2 \mathrm{~K}$ and the $T_{\mathrm{K}}$ subset by $\left\langle T_{\mathrm{ex}}\right\rangle=4.5 \pm 2.1 \mathrm{~K}$. The mean difference between the excitation temperature and kinetic temperature is $\left\langle T_{\mathrm{K}}-T_{\mathrm{ex}}\right\rangle=11.5 \pm 5.1 \mathrm{~K}$ for the full sample. This difference can be explained by a combination of the low volume-averaged densities detected and the effects of beam dilution. The densities are low enough that the $\mathrm{NH}_{3}$ transitions are not thermalized, as expected for the mean density we find (see D10 for more detail).

We can calculate an excitation density, defined to be the density which produces the observed level populations described by $T_{\mathrm{ex}}$ for the observed $T_{\mathrm{K}}$, from $T_{\mathrm{ex}}, T_{\mathrm{K}}$, and $\tau$ :

$$
n_{\mathrm{ex}}=\frac{k\left(J\left(T_{\mathrm{ex}}\right)-J\left(T_{\mathrm{cmb}}\right)\right)}{h v_{(1,1)}\left(1-J\left(T_{\mathrm{ex}}\right) / J\left(T_{\mathrm{K}}\right)\right)} n_{\mathrm{crit}} \beta,
$$

where

$$
J(T)=\frac{h v_{(1,1)}}{k\left(1-e^{-h v_{(1,1)} / k T}\right)},
$$

$T_{\mathrm{cmb}}=2.73 \mathrm{~K}$, the escape probability is given by $\beta=$ $\left(1-e^{-\tau}\right) / \tau$, where we take $\tau=\tau_{(1,1)} \times 0.233$, which is the maximum optical depth in a single hyperfine line of the $\mathrm{NH}_{3}(1,1)$ transition. Since $T_{\mathrm{ex}}$ will be lowered due to the beam dilution in the more distant sources while $T_{\mathrm{K}}$ is unaffected, the excitation density will be underestimated in sources suffering from beam dilution.

The excitation densities are listed in Column 7 of Table 7 . We cannot calculate $n_{\mathrm{ex}}$ for the sources where we set $T_{\mathrm{ex}}=T_{\mathrm{K}}$. The full sample has $\left\langle\log \left(\mathrm{n}_{\mathrm{ex}} / \mathrm{cm}^{-3}\right)\right\rangle=3.10 \pm 0.35$. The excitation density is plotted versus the volume-averaged $1.1 \mathrm{~mm}$ density in Figure 17 where black circles mark the $T_{\mathrm{K}}$ subsample and crosses mark BGPS sources without a $(2,2)$ detection. The dashed line marks a one-to-one correlation and is not a fit to the data. For the full sample, we find $\left\langle\log \left(n_{\mathrm{ex}} / n\right)\right\rangle=-0.02 \pm 0.58$ and a median of $10^{0.06}$, suggesting that both density estimates are reasonable.

\subsection{Column Densities and $\mathrm{NH}_{3}$ Abundance}

We calculate two measures of the $\mathrm{H}_{2}$ column density, the column density in a beam centered on the peak of the $1.1 \mathrm{~mm}$ emission $\left(N_{\mathrm{H}_{2}}^{\text {beam }}\right)$, and the average column density of the entire $1.1 \mathrm{~mm}$ source $\left(N_{\mathrm{H}_{2}}^{\mathrm{avg}}\right)$. The column density in a beam is calculated as

$$
N_{\mathrm{H}_{2}}^{\text {beam }}=\frac{S_{\nu}\left(40^{\prime \prime}\right)}{\Omega\left(40^{\prime \prime}\right) \mu_{\mathrm{H}_{2}} m_{\mathrm{H}} \kappa_{\nu} \mathrm{B}_{\nu}\left(T_{\mathrm{D}}\right)},
$$

where $S_{v}\left(40^{\prime \prime}\right)$ is the flux density in an aperture of diameter $40^{\prime \prime}$ without the aperture correction of 1.46 required to correct for point source emission that falls outside of the aperture, $\Omega\left(40^{\prime \prime}\right)=$ $2.95 \times 10^{-8} \mathrm{sr}$ is the solid angle of the $40^{\prime \prime}$ aperture, $\mu_{\mathrm{H}_{2}}=2.8$, $m_{\mathrm{H}}$ is the mass of a single hydrogen atom, $\kappa_{\nu}$ is the dust opacity described in Section 6.3, and $B_{v}\left(T_{\mathrm{D}}\right)$ is the Planck function evaluated at $T_{\mathrm{D}}$. We use $S_{v}\left(40^{\prime \prime}\right)$ as a measure of the average $1.1 \mathrm{~mm}$ surface brightness to measure $N_{\mathrm{H}_{2}}^{\text {beam }}$ rather than the single peak $1.1 \mathrm{~mm}$ pixel in order to reduce the effects of pixelto-pixel variations caused by the noise in the BGPS images. The solid angle subtended by the $40^{\prime \prime}$ aperture is very close to the solid angle of the $33^{\prime \prime}$ effective beam, $\Omega($ beam $)=2.9 \times 10^{-8} \mathrm{sr}$. We calculate $N_{\mathrm{H}_{2}}^{\text {avg }}$ as $M_{\text {iso }}$ (int) $/ \mu_{\mathrm{H}_{2}} m_{\mathrm{H}} \pi R^{2}$ and note that this quantity is typically smaller than $N_{\mathrm{H}_{2}}^{\text {beam }}$ since it is averaged over the entire area of the BGPS source while $N_{\mathrm{H}_{2}}^{\text {beam }}$ is calculated at the peak of the $1.1 \mathrm{~mm}$ emission. $N_{\mathrm{H}_{2}}^{\text {beam }}$ and $N_{\mathrm{H}_{2}}^{\text {avg }}$ are listed in Columns 9 and 10 of Table 7.

The average $\mathrm{H}_{2}$ column densities, $N_{\mathrm{H}_{2}}^{\text {avg }}$, are shown in Figure 12(d) versus kinematic distance. The gray circles denote the $T_{\mathrm{K}}$ subset, gray crosses denote sources without a $(2,2)$ detection, and the black squares and black solid line denote the mean $\mathrm{H}_{2}$ column densities in $2 \mathrm{kpc}$ bins. The dashed line denotes the $\mathrm{H}_{2}$ column density corresponding to the mass per beam limit described in Section 6.3. The dash-dot line marks a threshold gas surface density of $122.5 M_{\odot} \mathrm{pc}^{-2}$ above which efficient star formation occurs $\left(116 M_{\odot} \mathrm{pc}^{-2}\right.$, Lada et al. 2010; $129 M_{\odot} \mathrm{pc}^{-2}$, Heiderman et al. 2010), and the dotted line denotes a surface density of $1 \mathrm{~g} \mathrm{~cm}^{-2}$ required to prevent excessive fragmentation and to allow massive stars to form (Krumholz \& McKee 2008). See Section 8.4 for further discussion of these limiting column densities. The mean $\mathrm{H}_{2}$ column density decreases slightly with kinematic distance, but is essentially flat. The full sample is described by $\left\langle\log \left(N_{\mathrm{H}_{2}}^{\text {avg }} / \mathrm{cm}^{-2}\right)\right\rangle=21.81 \pm 0.27$ and $180 M_{\odot} \mathrm{pc}^{-2}$. Using $R_{V}=3.1$ and $N_{\mathrm{H}_{2}}^{\text {avg }} / A_{V}=9.4 \times 10^{20} \mathrm{~cm}^{-2} \mathrm{mag}^{-1}$ (Bohlin et al. 1978), the mean $N_{\mathrm{H}_{2}}^{\text {avg }}$ for the full sample corresponds to $8.5 \pm 6.8 \mathrm{mag}$ of visual extinction. Assuming an $R_{V}=5.5$ extinction model (Weingartner \& Draine 2001), $N_{\mathrm{H}_{2}}^{\text {avg }} / A_{V}=6.86 \times 10^{20} \mathrm{~cm}^{-2} \mathrm{mag}^{-1}$, the mean of the full sample corresponds to $11.7 \pm 9.3 \mathrm{mag}$.

We calculate the $\mathrm{NH}_{3}$ column densities as described in D10 and list them in Column 11 of Table 7. Statistical properties are listed in Table 8 .

The $\mathrm{NH}_{3}$ column densities are described by $\left\langle\log \left(N_{\mathrm{NH}_{3}} /\right.\right.$ $\left.\left.\mathrm{cm}^{-2}\right)\right\rangle=14.75 \pm 0.37$ and $\left\langle\log \left(N_{\mathrm{NH}_{3}} / \mathrm{cm}^{-2}\right)\right\rangle=14.95 \pm 0.29$ for the full sample and $T_{\mathrm{K}}$ subsample, respectively. We additionally find a mean $\mathrm{NH}_{3}$ abundance of $\left\langle\log \left(N_{\mathrm{NH}_{3}} / N_{\mathrm{H}_{2}}^{\text {beam }}\right)\right\rangle=$ $-7.34 \pm 0.03$ for the full sample. Our measured $\mathrm{NH}_{3}$ abundance is consistent with other studies: $3 \times 10^{-8}$ (Harju et al. 1993), $2.8 \times 10^{-8}$ (Tafalla et al. 2006), and $2 \times 10^{-8}$ (Foster et al. 2009). 


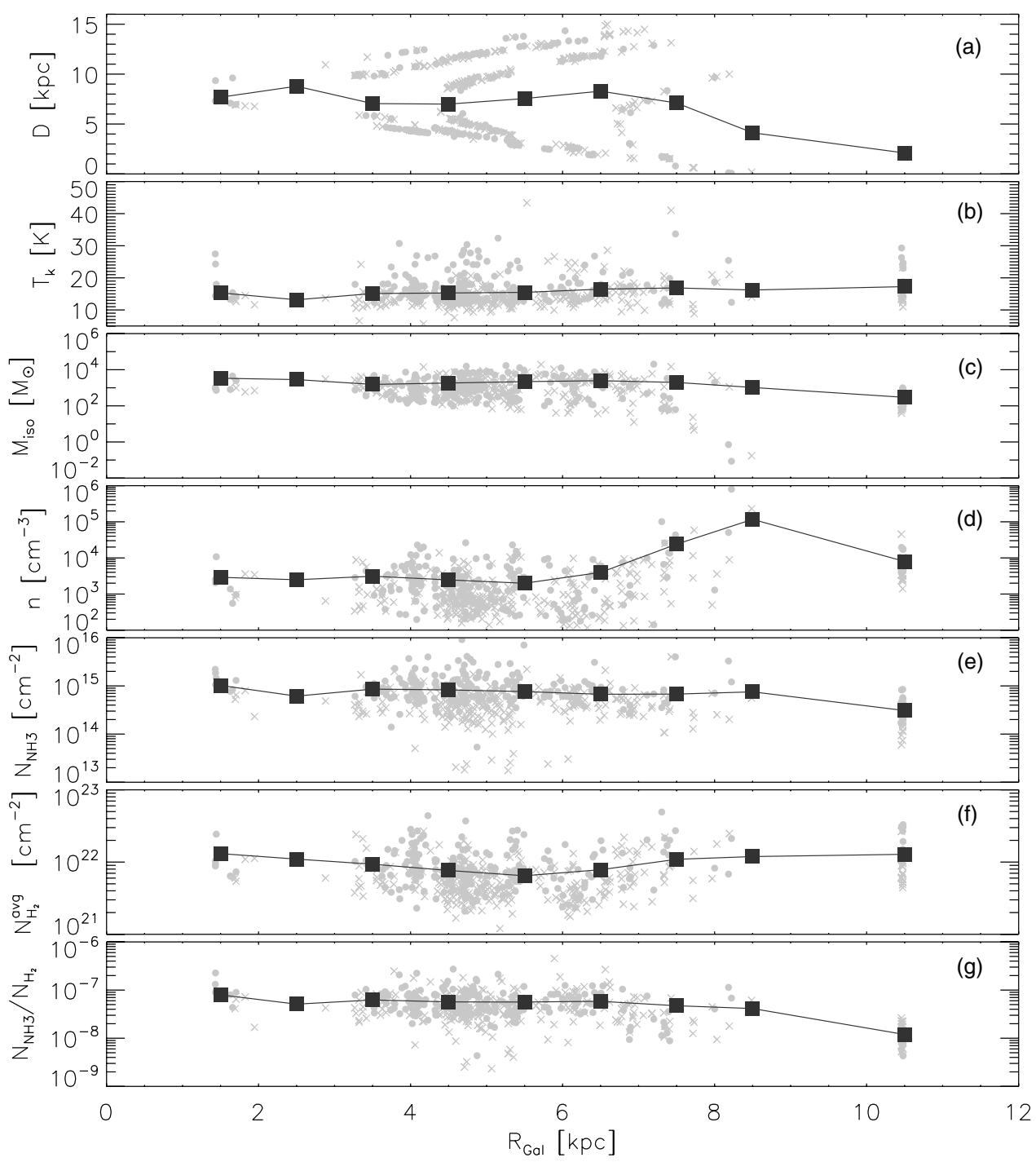

Figure 18. Kinematic distance, $T_{\mathrm{K}}, M_{\mathrm{iso}}, n, N_{\mathrm{NH}_{3}}, N_{\mathrm{H}_{2}}^{\text {avg }}$, and $\mathrm{NH}_{3}$ abundance vs. Galactocentric radius. Gray circles mark the $T_{\mathrm{K}}$ subsample, gray crosses denote sources without a $(2,2)$ detection, black squares mark the mean of each property within $2 \mathrm{kpc}$ wide bins.

\section{GALACTIC TRENDS IN PHYSICAL PROPERTIES}

As discussed in Section 5, each BGPS source has a unique $R_{\text {Gal }}$ given by the observed radial velocity and assumed Galactic rotation curve, allowing us to study the trends in physical properties as a function of distance from the Galactic center. In this section we include the Gemini OB1 molecular cloud (Gem OB1) sources from D10 as an outer Galaxy data point. Since the BGPS did not perform a blind survey of the outer Galaxy, but rather targeted known star-forming regions (Aguirre et al. 2011), the Gem OB1 sources may be biased in terms of evolutionary stage. We discuss possible effects of this bias in Section 7.2. We also note that the Gem OB1 sources may not be representative of all outer Galaxy star formation.

Figure 18 plots the physical properties as a function of Galactocentric radius for the $T_{\mathrm{K}}$ subsample (gray circles) and the BGPS sources without a $(2,2)$ detection (gray crosses). The black squares denote the mean properties within $2 \mathrm{kpc}$ bins centered at 1, 3, 5, 7, 9, and $11 \mathrm{kpc}$. We observe BGPS sources at both the near and far kinematic distances for each Galactocentric radius. The concentration of BGPS sources in lines in
Figure 18(a) is due to the range of Galactic longitudes included in the full sample. Each sideways V formation corresponds to one of the four ranges in Galactic longitude, where the vertex of the $\mathrm{V}$ created by the $\ell \sim 9^{\circ}$ sources is at the smallest $R_{\mathrm{Gal}}$ and the $\ell \sim 54^{\circ}$ sources are found at $R_{\text {Gal }} \sim 7 \mathrm{kpc}$.

The observed dichotomy of kinematic distance as a function of Galactocentric radius causes a spread in physical properties such as $M_{\text {iso }}, n$, and $\mathrm{N}_{\mathrm{H}_{2}}$ as a function of $R_{\mathrm{Gal}}$ because of their dependence on the kinematic distance (see Figure 12). This spread in properties will serve to mask any trends in properties as a function of $R_{\mathrm{Gal}}$. In this section, we consider the near subsample (233 BGPS sources placed at the near kinematic distance) in order to remove the ambiguity introduced by the kinematic distance dichotomy.

The mean and standard error in the mean are presented for each property for the near subsample in Figure 19. The 233 BGPS sources were sorted by $R_{\mathrm{Gal}}$ and split evenly into six bins with 39 sources in the first five bins and 38 sources in the sixth bin. The 33 Gem OB1 sources are presented separately in the seventh bin. This binning method provides more measurements of the mean properties where there are more BGPS sources. 

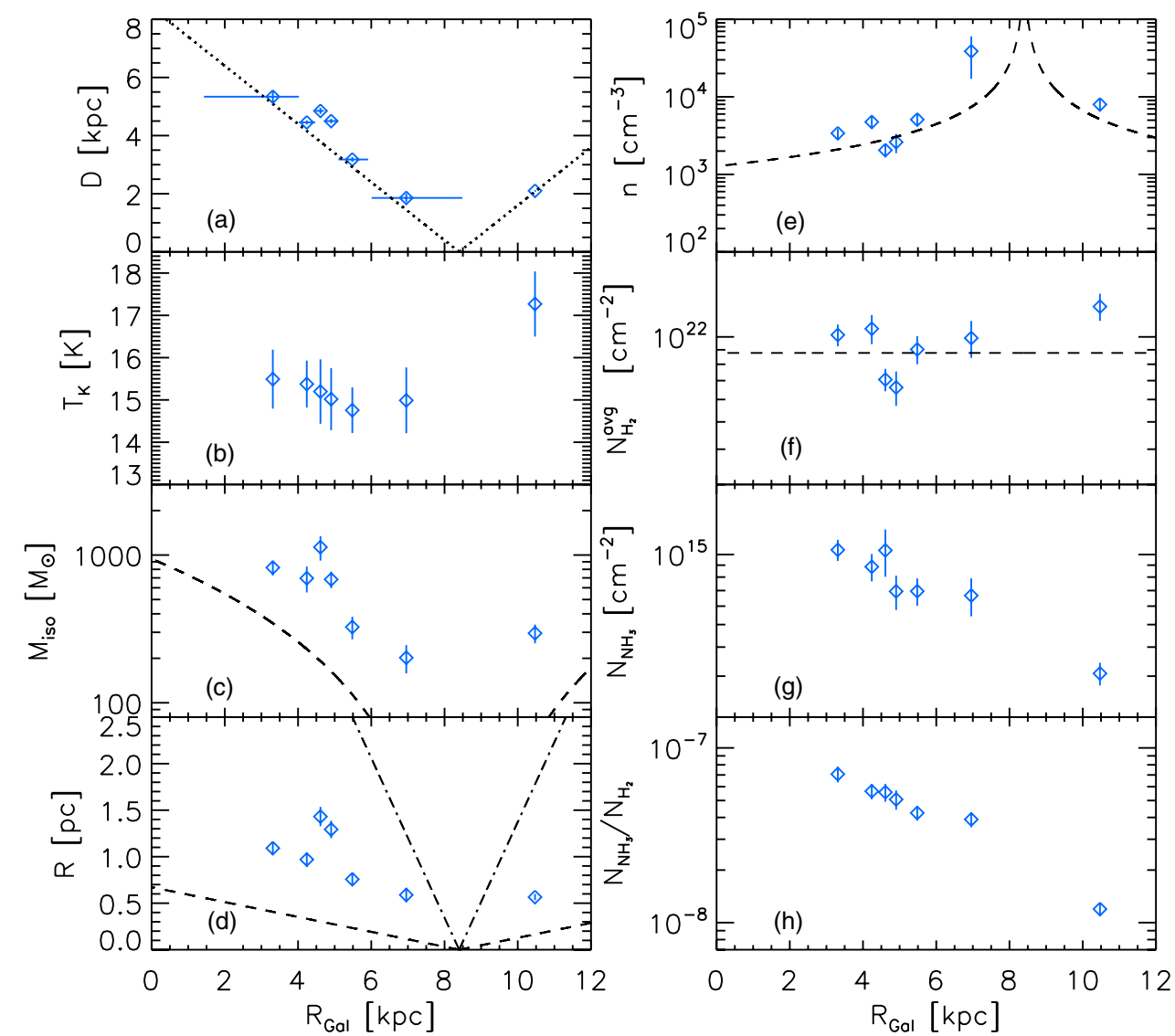

Figure 19. Mean properties of near BGPS sources vs. Galactocentric radius for (a) kinematic distance, (b) $T_{\mathrm{K}}$, (c) $M_{\mathrm{iso}}$, (d) $R$, (e) $n$, (f) $N_{\mathrm{H}_{2}}^{\text {avg }}$, (g) $N_{\mathrm{NH}_{3}}$, and (h) $N_{\mathrm{NH}_{3}} / N_{\mathrm{H}_{2}}^{\text {beam }}$. Each point represents the mean over 39 BGPS sources, except for the last two points, which represent 38 and 33 sources, respectively. The points are centered at the mean $R_{\text {Gal }}$ of 3.3, 4.2, 4.6, 4.9, 5.5, 7.0, and $10.5 \mathrm{kpc}$. The blue diamonds and vertical lines denote the mean and standard error in the mean (given by $\sigma / \sqrt{N}$ where $\sigma$ is the standard deviation and $N$ is the number of sources per bin) for each property. The blue horizontal lines in panel (a) represent the range of $R_{\mathrm{Gal}}$ included in each point. The dotted line in panel (a) represents the distance to the nearest source at a given $R_{\mathrm{Gal}}$, which corresponds to the $\ell=0^{\circ}$ line of sight. The dashed lines represent: (c) the $50 \sigma$ mass beam ${ }^{-1}$, (d) beam size, which represents a lower limit on extracted source size, and the scale above which we filter out uniform emission representing an upper limit to extracted source size (5.9, dash-dot line), (e) the $5 \sigma$ volume-averaged density per beam, and (g) the $50 \sigma$ average column density per beam.

(A color version of this figure is available in the online journal.)

The seven bins are centered at $R_{\mathrm{Gal}}$ of $3.3,4.2,4.6,4.9,5.5$, 7.0 , and $10.5 \mathrm{kpc}$. Bins $2-4$, centered at $4.2,4.6$, and $4.9 \mathrm{kpc}$, represent sources in the $5 \mathrm{kpc}$ molecular ring. The blue diamonds represent the mean properties within each bin and the vertical blue lines represent the standard error in the mean, which is given by $\sigma / \sqrt{N}$ where $\sigma$ is the standard deviation and $N$ is the number of sources in each bin.

Figure 19(a) plots the mean kinematic distance versus $R_{\mathrm{Gal}}$. The horizontal blue lines depict the range of $R_{\mathrm{Gal}}$ values included in each bin. The dotted lines correspond to the distance to the nearest source at a given $R_{\mathrm{Gal}}$, which lies along $\ell=0^{\circ}$ between the Sun and the Galactic center. The near subsample decreases in kinematic distance as $R_{\mathrm{Gal}}$ increases such that sources at higher $R_{\text {Gal }}$ are at smaller kinematic distances. The kinematic distances are similar to the nearest source distance shown by the dotted lines, allowing us to apply our understanding of the biases as described in Sections 6 and 8.2 to the near subsample as a function of $R_{\mathrm{Gal}}$.

\subsection{Observed Trends}

Here we highlight the observed trends in physical properties with $R_{\mathrm{Gal}}$, and discuss the possible biases in the following section.
The sources within the molecular ring are located at larger kinematic distances than expected given the smooth trend of decreasing kinematic distance with increasing $R_{\text {Gal }}$ seen in the inner Galaxy sources located outside of the molecular ring (see Figure 19(a)). We will explore the possible effects of the larger kinematic distance toward the molecular ring sources in the following section.

Figure 19(b) shows the kinetic gas temperature derived from the $\mathrm{NH}_{3}$ observations. There is no real trend in $T_{\mathrm{K}}$ of sources in the inner Galaxy, but there is a $3 \mathrm{~K}$ difference between the mean $T_{\mathrm{K}}$ of the inner Galaxy and Gem OB1 sources.

$M_{\text {iso }}$ is shown in Figure 19(c). The dashed lines depict a $50 \sigma$ mass per beam at distances corresponding to the dotted lines in panel (a). $M_{\text {iso }}$ steadily decreases with $R_{\text {Gal }}$ with the trend roughly following the shape of the curve representing a $50 \sigma$ mass per beam. The three bins closest to the molecular ring at $4.2,4.6$, and $4.9 \mathrm{kpc}$ deviate from the general trend in decreasing $M_{\text {iso }}$ with $R_{\text {Gal }}$ and are roughly a factor of a few more massive than described by the general decreasing trend.

Figure 19(d) displays the mean observed physical radius in each bin. The dashed lines mark the beam size at distances corresponding to the dotted lines in panel (a), which represent a minimum source size, and the dash-dot lines mark the upper limit in source size above which the data reduction 
filters out emission. The mean radius decreases with $R_{\mathrm{Gal}}$, but two of the three bins representing the molecular ring have radii approximately 1.5 times that expected from the general decreasing trend.

The volume-averaged density is shown in Figure 19(e) as a function of $R_{\mathrm{Gal}}$. The dashed lines mark the $5 \sigma$ density per beam required for a detection in the BGPS. Two of the bins representing the molecular ring fall below this line while the other points lie above.

Figure 19(f) plots $\mathrm{N}_{\mathrm{H}_{2}}^{\text {avg }}$ as a function of $R_{\mathrm{Gal}}$. The average $\mathrm{H}_{2}$ column density is roughly constant with $R_{\mathrm{Gal}}$, with the lowest column densities seen in the molecular ring and the highest in Gem OB1. The dashed line denotes a $50 \sigma$ column density per beam. The observed $\mathrm{NH}_{3}$ column density is shown in Figure $19(\mathrm{~g})$ as a function of $R_{\mathrm{Gal}}$. The column density decreases with $R_{\text {Gal }}$ and only one of the molecular ring bins falls below the general trend. Figure 19(h) plots the observed $\mathrm{NH}_{3}$ abundance versus $R_{\mathrm{Gal}}$. The abundance, given by $N_{\mathrm{NH}_{3}} / N_{\mathrm{H}_{2}}^{\text {beam }}$, decreases with $R_{\mathrm{Gal}}$ and is approximately a factor of seven higher in the inner Galaxy than in the Gem OB1 sources.

\subsection{Biases and Implications}

As mentioned above, the Gem OB1 sources belong to a molecular cloud known to be forming stars. This could bias the physical properties, thereby providing an inappropriate comparison point for the inner Galaxy sources. The Gem OB1 sources differ dramatically from the inner Galaxy sources in only two properties: $T_{\mathrm{K}}$ and $\mathrm{NH}_{3}$ abundance. The warmer mean $T_{\mathrm{K}}$ observed in the Gem OB1 sources could be due to a bias toward later evolutionary stages, which typically have warmer dust temperatures (Battersby et al. 2010). Similarly, $T_{\mathrm{K}}$ of BGPS sources increases with the number of associated mid-IR sources (see Section 8.3). By targeting a region of known star formation, we have potentially excluded the earliest evolutionary stages that have colder temperatures, therefore resulting in a warmer mean $T_{\mathrm{K}}$.

Foster et al. (2009) found no significant difference between the $\mathrm{NH}_{3}$ abundance of starless and protostellar cores in Perseus, suggesting that the potential bias of the Gem OB1 sources to later evolutionary states is not likely to be the cause of the lower $\mathrm{NH}_{3}$ abundance in Gem OB1 sources. The general decrease in $\mathrm{NH}_{3}$ abundance (Figure 18(h)) could be the result of changes in dust properties and/or metallicity with $R_{\mathrm{Gal}}$. Changes in the gas-to-dust ratio, grain size distribution, and temperature of dust grains across the Galaxy could all result in the observed decrease in $\mathrm{NH}_{3}$ abundance with $R_{\mathrm{Gal}}$. Similarly, a decrease with $R_{\mathrm{Gal}}$ in the amount of nitrogen available to form $\mathrm{NH}_{3}$ would result in a lower abundance at larger $R_{\mathrm{Gal}}$. The decrease we observe mimics the decreasing nitrogen abundance seen as a function of $R_{\mathrm{Gal}}$ (e.g., Gummersbach et al. 1998; Shaver et al. 1983; Smartt et al. 2001; Rolleston et al. 2000). We fit a line to the $\mathrm{NH}_{3}$ abundance as a function of $R_{\mathrm{Gal}}$ and find

$$
X_{\mathrm{NH}_{3}}=10^{-6.90 \pm 0.05} 10^{-0.096 \pm 0.008} R_{\mathrm{Gal}}
$$

We observe a gradient of $-0.096 \mathrm{dex} \mathrm{kpc}^{-1}$ in the $\mathrm{NH}_{3}$ abundance, which agrees within the error bars with the observed nitrogen gradient $\left(-0.09 \pm 0.015 \mathrm{dex} \mathrm{kpc}{ }^{-1}\right.$; Shaver et al. 1983). Thus, the $\mathrm{NH}_{3}$ abundance decreases at least as quickly as the nitrogen abundance but possibly faster due to possible changes in dust properties to which our observations are not sensitive. This trend needs to be taken into account when calculating $\mathrm{H}_{2}$ column densities and masses based on observed $\mathrm{NH}_{3}$ column densities.

The effects of distance must also be taken into account when interpreting trends with $R_{\mathrm{Gal}}$. The Malmquist bias together with the spatial filtering in the BGPS data has resulted in the detection of different types of structures at different distances (i.e., cores, clumps, clouds; see Section 8.2), which complicates the study of BGPS sources as a function of position in the Galaxy. This complication, which will affect all continuum studies, needs to be addressed carefully.

Both the mass and radius of BGPS sources exhibit the same general trend of decreasing values with increasing $R_{\mathrm{Gal}}$ with peaks within the molecular ring. For the near subsample, $R_{\mathrm{Gal}}$ and $D$ are inversely proportional so that large values of $R_{\mathrm{Gal}}$ correspond to small kinematic distances and small $R_{\mathrm{Gal}}$ corresponds to large kinematic distances. Therefore, the larger masses and radii seen at low $R_{\mathrm{Gal}}$ are likely the result of the Malmquist bias. Indeed, the slope of the mean mass versus $R_{\mathrm{Gal}}$ roughly follows the slope of the $50 \sigma$ mass per beam line suggesting that trend is caused by distance effects.

The general trends of decreasing masses and radii with increasing $R_{\mathrm{Gal}}$ are due to distance effects, but are the larger masses and radii seen toward molecular ring sources real? The difference in mean masses and radii plotted in Figure 19 suggests that the molecular ring sources are truly larger and more massive, but the sources with $R_{\mathrm{Gal}}=4-5 \mathrm{kpc}$ are also located at larger distances than described by the general trend of decreasing kinematic distance with $R_{\mathrm{Gal}}$ (Figure 19(a)). We explore the possible effects of distance by comparing properties of sources inside ( $4 \leqslant R_{\mathrm{Gal}} \leqslant 5 \mathrm{kpc} ; 115$ BGPS sources) and outside the molecular ring (151 BGPS sources) using Kolomogorov-Smirnov (K-S) tests. The two-sided K-S test compares the cumulative distribution functions of two samples and measures the probability that the sources are drawn from the same parent distribution, but high $\mathrm{K}-\mathrm{S}$ probabilities do not necessarily indicate the same parent population. Figure 20 plots the distributions of kinematic distance (upper panel), physical radius (lower left panel), and $M_{\text {iso }}$ (lower right panel) for sources within the molecular ring (solid line histogram) and sources outside the molecular ring (light gray histogram). The K-S probability for the kinematic distance $\left(10^{-26}\right)$ shows that we are not comparing similar distance distributions by splitting the sample into sources found inside the molecular ring and those found outside the molecular ring. The sources located outside the molecular ring span a large range of kinematic distances, as seen in the upper panel of Figure 20. We have attempted to further untangle the effects of distance by considering only the sources inside and outside the molecular ring within the same range of kinematic distances, specifically $3.5 \leqslant D \leqslant 6.5 \mathrm{kpc}$. This distance range includes 114 of the 115 sources inside the molecular ring, and 42 of the 151 sources outside the molecular ring (dark gray histogram in Figure 20). We performed similar K-S tests on these kinematic distance selected samples and found K-S probabilities of $0.41,0.081$, and 0.036 for $D, R$, and $M_{\text {iso }}$, respectively. The K-S probabilities have increased compared to the non-kinematic-distance-selected sample and no longer suggest measurable differences for any of these properties.

Therefore, the larger mean masses and radii of sources within the molecular ring are likely caused by distance effects. When we compare sources at similar kinematic distances, we see that there is little variation in physical properties regardless of environment (inside versus outside the molecular ring, for 

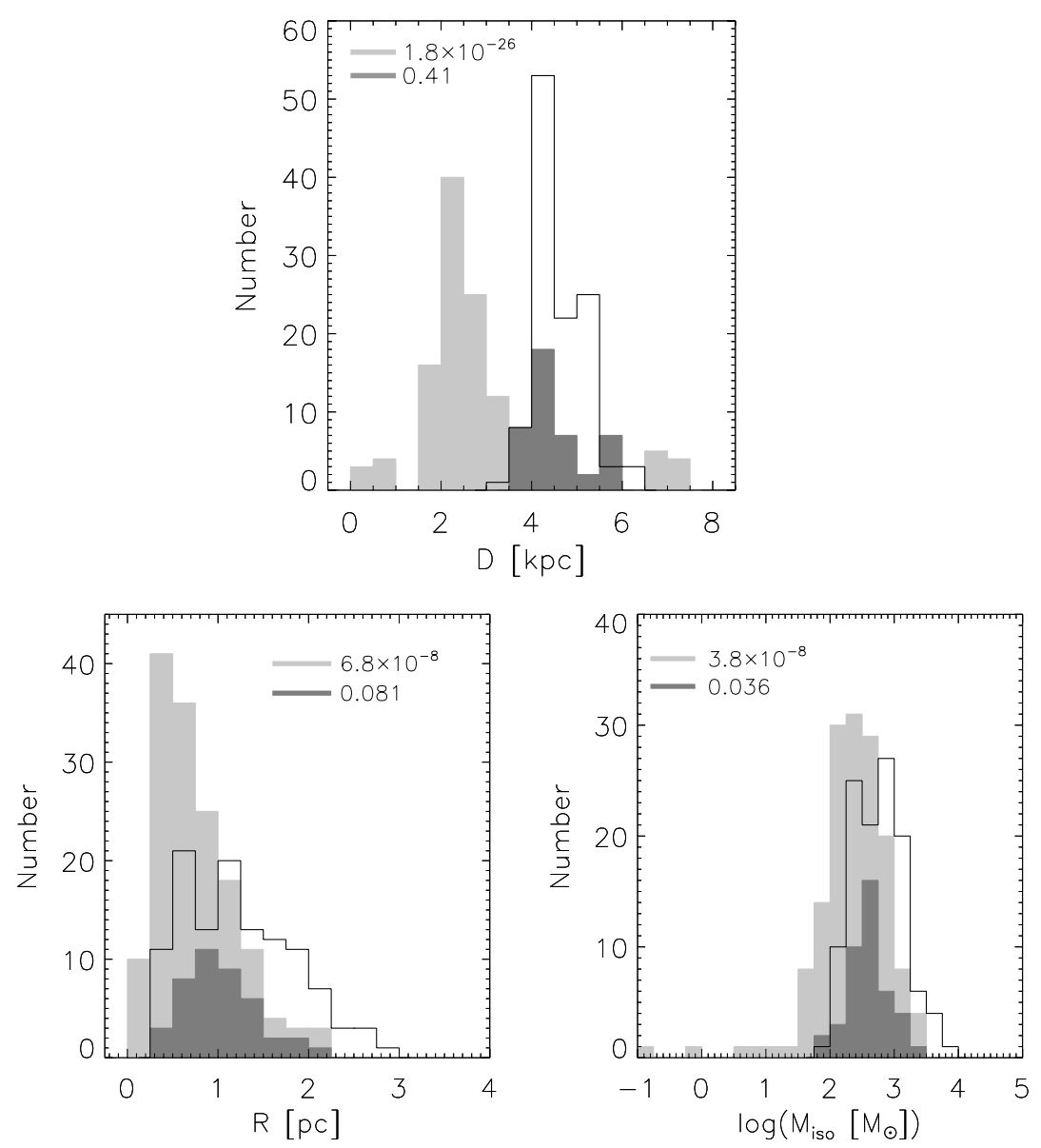

Figure 20. Distributions of kinematic distance (upper panel), physical radius (lower left panel), and $M_{\text {iso }}$ (int) (lower right panel) for the near kinematic distance sources located within the molecular ring (solid line), outside the molecular ring (light gray), and outside the molecular ring with $3.5 \mathrm{kpc} \leqslant D \leqslant 6.5 \mathrm{kpc}$ (dark gray). The legend gives the K-S probabilities comparing the full sample of sources inside and outside of the molecular ring (light gray) and for the kinematic-distance-selected samples of sources inside and outside of the molecular ring (dark gray).

example). This is in contrast to the molecular clouds seen in the ${ }^{13} \mathrm{CO}$ GRS survey. The molecular clouds within the molecular ring typically have warmer temperatures, larger areas, larger masses, higher peak $\mathrm{H}_{2}$ column densities, and typically contain more clumps than molecular clouds located outside the molecular ring (Rathborne et al. 2009). This suggests that environment may affect the large-scale molecular clouds while the regions of dense gas $\left(n \sim 10^{3} \mathrm{~cm}^{-3}\right)$ within the molecular clouds exhibit similar properties regardless of environment.

\section{DISCUSSION}

\subsection{Comparison to Other Surveys}

Schlingman et al. (2011) conducted a survey of BGPS sources in $\mathrm{HCO}^{+}(3-2)$ and $\mathrm{N}_{2} \mathrm{H}^{+}(3-2)$ and were able to break the kinematic distance ambiguity for 648 sources that they placed at the near distance. They find a median radius of $0.75 \mathrm{pc}$, median isothermal mass assuming $T_{\mathrm{D}}=20 \mathrm{~K}$ of $330 \mathrm{M}_{\odot}$, and a median volume-averaged density of $2.5 \times 10^{3} \mathrm{~cm}^{-3}$. For the $233 \mathrm{BGPS}$ sources placed at the near kinematic distance in our sample, we find a median radius of $0.95 \mathrm{pc}$, median mass of $350 M_{\odot}$, and a median volume-averaged density of $2.2 \times 10^{3} \mathrm{~cm}^{-3}$, very similar to the properties of the Schlingman et al. sample. In contrast to our sample, where we find a median virial parameter of 0.74 , Schlingman et al. find typical virial parameters of a few and only a small number of sources have virial parameters less than one. This difference is likely due to the larger line widths detected in $\mathrm{HCO}^{+}$(median FWHM $=2.98 \mathrm{~km} \mathrm{~s}^{-1}$ ) compared to the line widths detected in $\mathrm{NH}_{3}$ (median $\mathrm{FWHM}=0.64 \mathrm{~km} \mathrm{~s}^{-1}$ $\sqrt{8 \ln 2}=1.5 \mathrm{~km} \mathrm{~s}^{-1}$ ).

The APEX Telescope Large Area Survey of the Galaxy (ATLASGAL; Schuller et al. 2009) has surveyed the Galactic plane at $870 \mu \mathrm{m}$. Early results from this survey and $\mathrm{NH}_{3}$ followup observations reveal similar physical properties to those presented in this work. For 24 ATLASGAL sources near $\ell \sim 19^{\circ}$ that were detected in $\mathrm{NH}_{3}$, Schuller et al. find $T_{\mathrm{K}}$ ranging from 14 to $33 \mathrm{~K}$, masses ranging from roughly $10^{2}$ to $10^{4} M_{\odot}$, and $\mathrm{H}_{2}$ column densities from $7 \times 10^{21} \mathrm{~cm}^{-2}$ to $1.3 \times 10^{22} \mathrm{~cm}^{-2}$. The range of properties of ATLASGAL sources is consistent with the range of properties of BGPS sources presented here.

Compared to the GRS clouds studied by Roman-Duval et al. (2009), the BGPS sources are smaller (BGPS $R=$ 0.01-8.5 pc; GRS $R=0.1-40 \mathrm{pc}$ ), less massive (BGPS $M_{\text {iso }}=0.1-2.4 \times 10^{4} M_{\odot} ;$ GRS $\left.M=10-10^{6} M_{\odot}\right)$, higher density (BGPS $\langle n\rangle=5 \times 10^{3} \mathrm{~cm}^{-3}$; GRS $\langle n\rangle=230 \mathrm{~cm}^{-3}$ ), and have higher surface densities (BGPS $\langle\Sigma\rangle=180 M_{\odot} \mathrm{pc}^{-2}$; GRS $\left.\langle\Sigma\rangle=144 M_{\odot} \mathrm{pc}^{-2}\right)$. This is consistent with the paradigm that the BGPS sources are typically the smaller, denser regions located within molecular clouds.

\subsection{What is a BGPS Source?}

D10 addressed the question "what is a BGPS source" in terms of expected masses and densities as a function of distance based 


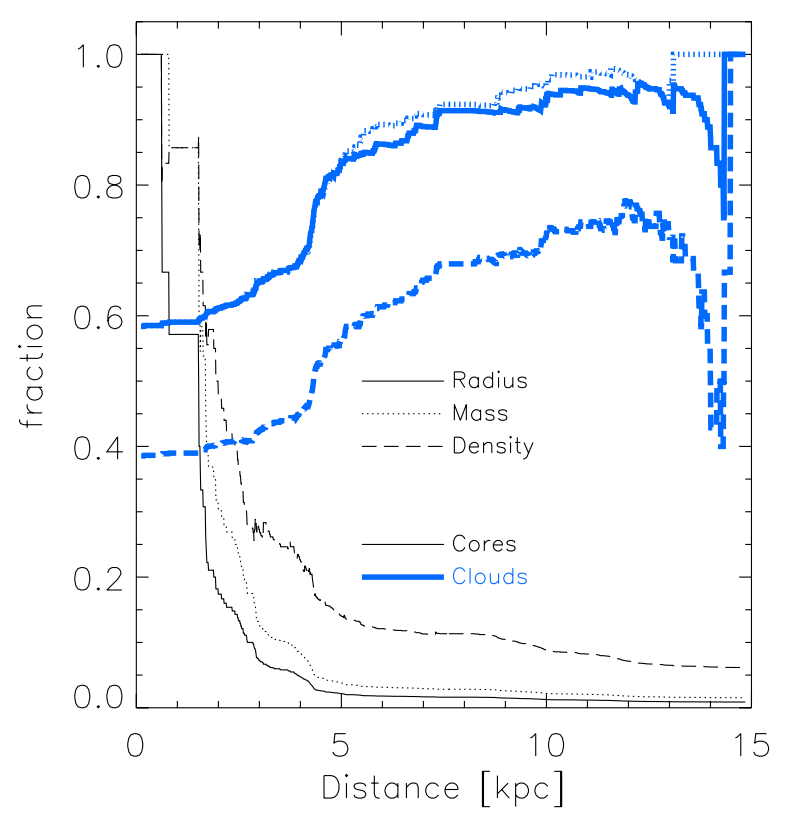

Figure 21. Fraction of sources in the full sample of 456 BGPS sources detected in $\mathrm{NH}_{3}$ nearer that are cores (thin black lines) and farther that are clouds (thick blue lines) as a function of kinematic distance determined by radius (solid lines), mass (dotted lines), and volume-averaged density (dashed lines). Both ends of the distance range suffer from small number statistics. See Section 8.2 for further details.

(A color version of this figure is available in the online journal.)

on sources at a single, well-known distance. With a large sample spanning a large range of kinematic distances, we can address what type of source the BGPS is detecting at each distance empirically. As discussed throughout Section 6, BGPS sources can be classified as clouds, clumps, or cores based on their size, mass, and volume-averaged density. While there can be significant overlap in the physical properties of clouds, clumps, and cores, they can be approximately separated by the dotted lines shown in Figures 12(a)-(c).

Figure 21 shows the fraction of sources closer than a given distance that are described as cores (thin black lines; $R \leqslant$ $0.125 \mathrm{pc}, M_{\text {iso }} \leqslant 27.5 M_{\odot}$, and $n \geqslant 10^{4} \mathrm{~cm}^{-3}$ ) based on radius (solid), mass (dotted), and volume-averaged density (dashed). We find that greater than $90 \%$ of the BGPS sources out to a distance of $0.68 \mathrm{kpc}$ are well described as cores. However, we suffer from small number statistics at distances less than $2 \mathrm{kpc}$ (see Figure 9), and the distance out to which we detect cores could be greater. Since no sources are detected between 1 and approximately $1.75 \mathrm{kpc}$, the fraction of BGPS sources that are cores cannot be characterized in this distance range. Thus, this result is consistent with D10, who determined that cores are detected out to approximately $2 \mathrm{kpc}$.

Similarly, we determine the fraction of sources located farther than a given distance that are characterized as clouds $(R \geqslant$ $1.25 \mathrm{pc}, M_{\text {iso }} \geqslant 750 M_{\odot}$, and $n \leqslant 750 \mathrm{~cm}^{-3}$ ). These fractions are shown in Figure 21 as the thick blue lines. The fraction of sources with volume-averaged densities below the limit for clouds never reaches 0.9 , but does follow the general trends seen in the fractions based on radius and mass. The density curve never reaches $90 \%$ because it is suffering from effects of volume averaging. Thus, we do not consider the volumeaveraged density when determining the distance at which we detect $\geqslant 90 \%$ clouds. We find $7.3 \mathrm{kpc}$ based on the fraction with radii $\geqslant 1.25 \mathrm{kpc}$ and $6.6 \mathrm{kpc}$ from the fraction with $M_{\text {iso }}($ int $) \geqslant 750 M_{\odot}$. We take the average of these two distances,
Table 9

Trends with Star Formation Activity

\begin{tabular}{lcrrrrr}
\hline \hline \multirow{2}{*}{$\begin{array}{l}\text { Property } \\
\text { units) }\end{array}$} & Probability & \multicolumn{5}{c}{ Standard } \\
\cline { 3 - 7 }$\sigma_{\text {Vlsr }}$ & Group & Minimum & Mean & Deviation & Median & Maximum \\
$\left(\mathrm{km} \mathrm{s}^{-1}\right)$ & 0 & 0.1 & 0.7 & 0.4 & 0.6 & 3.6 \\
& 1 & 0.2 & 0.7 & 0.5 & 0.6 & 4.4 \\
& 2 & 0.2 & 0.7 & 0.5 & 0.7 & 3.8 \\
$T_{\mathrm{K}}$ & 3 & 0.3 & 1.1 & 0.5 & 1.0 & 3.1 \\
$(\mathrm{~K})$ & 0 & 6.6 & 13.9 & 3.0 & 13.5 & 26.6 \\
& 1 & 5.0 & 14.6 & 3.8 & 13.8 & 33.7 \\
& 2 & 10.3 & 15.8 & 3.9 & 14.6 & 28.6 \\
$N_{\mathrm{NH}}$ & 3 & 11.1 & 22.7 & 8.2 & 21.1 & 59.4 \\
$\left(10^{14} \mathrm{~cm}^{-2}\right)$ & 1 & 0.2 & 5.6 & 3.8 & 4.6 & 21.1 \\
& 2 & 0.2 & 7.1 & 5.5 & 5.8 & 40.2 \\
& 3 & 0.2 & 8.0 & 6.6 & 6.1 & 40.0 \\
& & 2.0 & 16.3 & 16.9 & 10.4 & 91.2 \\
\hline
\end{tabular}

$7.0 \mathrm{kpc}$, as the distance above which the BGPS detects $\geqslant 90 \%$ clouds.

At distances up to approximately $1 \mathrm{kpc}$, the BGPS detects cores. At distances greater than approximately $7 \mathrm{kpc}$, the BGPS detects clouds. At intermediate distances, the BGPS will detect predominately clumps, but also cores and clouds. While the high column density features seen in the BGPS do identify regions of dense gas, they represent very different scales of structures when looking through the Galaxy. Similar continuum based studies (e.g., ATLASGAL, Schuller et al. 2009; Hi-GAL, Molinari et al. 2010) will also be subject to these effects. Kinematic data are crucial in interpreting the results of such studies and determining what structures are actually observed.

\subsection{Star Formation Activity}

Dunham et al. (2011) characterized the star formation activity of the BGPS sources based on the presence of mid-infrared (midIR) sources along a line of sight coincident with each BGPS source. They placed each BGPS source in one of four groups representing increasing confidence in the type of associated mid-IR source reliably indicating star formation activity (as opposed to a chance alignment). Group 3 represents the highest confidence in star formation activity and includes BGPS sources matched with an Extended Green Object (EGO; Cyganowski et al. 2008) or RMS (Hoare et al. 2004; Urquhart et al. 2008) source. Groups 1 and 2 include BGPS sources matched with GLIMPSE red sources cataloged by Robitaille et al. (2008) or a deeper GLIMPSE red source catalog created by Dunham et al. (2011). Group 0 includes BGPS sources that were not matched with any mid-IR sources, and we refer to them as "starless" although they are not necessarily truly starless (see Dunham et al. 2011 for more details).

Dunham et al. (2011) find that $49 \%$ of BGPS sources in the inner Galaxy where all surveys overlap contain at least one midIR source. We find that $67 \%$ of the full sample contains at least one mid-IR source, while $33 \%$ are "starless." Sixty-eight percent of the BGPS sources without a detection in the $\mathrm{NH}_{3}(1,1)$ line were "starless," and groups 1 through 3 comprise roughly $21 \%$, $9 \%$, and $3 \%$, respectively. The BGPS sources without $\mathrm{NH}_{3}$ or any corresponding mid-IR sources may be false detections in the BGPS catalog.

Figure 22 plots the fraction of BGPS sources in the near subsample that contain at least one mid-IR source as a function of kinematic distance (left panel). The first five data points 

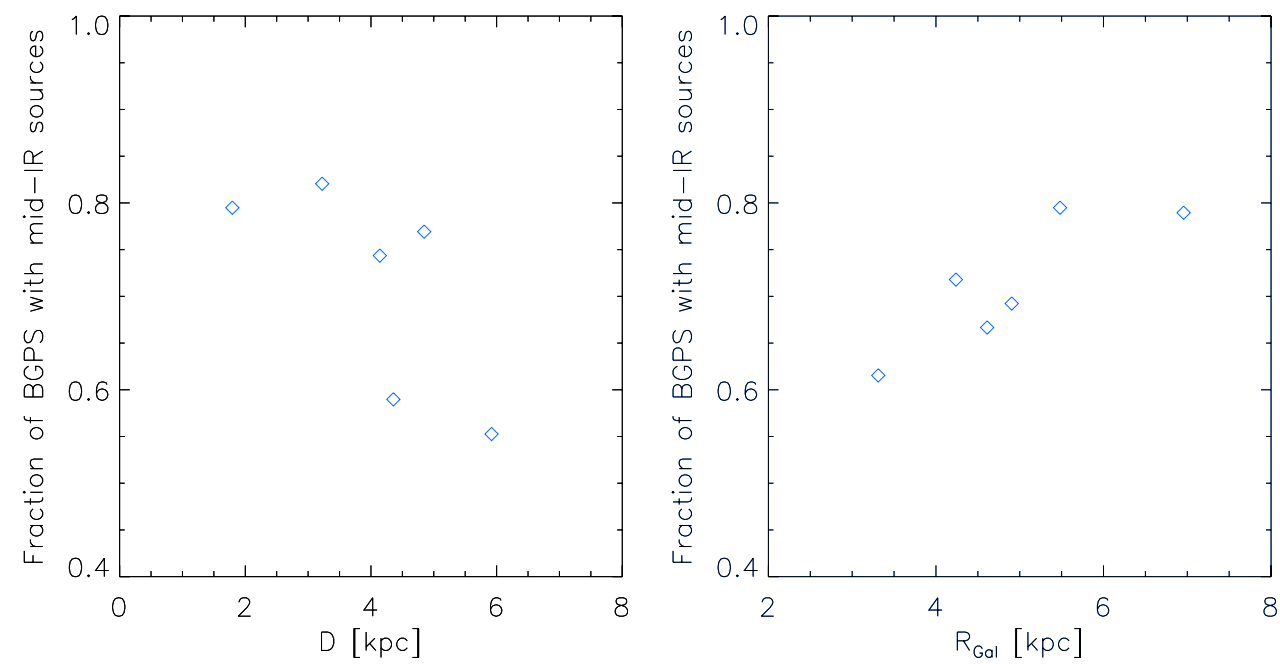

Figure 22. Fraction of BGPS sources associated with at least one mid-IR source (Dunham et al. 2011) as a function of kinematic distance (left) and $R_{\mathrm{Gal}}$ (right) for the near subsample of 233 BGPS sources placed at the near kinematic distance. Each diamond includes 39 BGPS sources, except for the bin at $R_{\mathrm{Gal}} \sim 7 \mathrm{kpc}$, which represents 38 BGPS sources.

(A color version of this figure is available in the online journal.)

represent 39 BGPS sources and the sixth represents 38 . By placing the same number of BGPS sources in each bin, we obtain more data points at distances where more BGPS sources are located. The fraction peaks near $85 \%$ at a distance of approximately $3.0 \mathrm{kpc}$ and reaches a minimum of $55 \%$ at $6 \mathrm{kpc}$. The decrease in fraction of BGPS sources with mid-IR sources is likely a result of the increasing distances. At the farthest distances shown here, the BGPS is detecting the large-scale objects, clumps or clouds, and the GLIMPSE and RMS surveys would only detect the most luminous YSOs, which become increasingly rare with increasing luminosity and mass. Thus, the decreasing fraction we see with distance may simply be a reflection of a decreasing number of detected YSOs in the midIR catalogs. The fraction of BGPS sources with mid-IR sources also reaches a minimum at kinematic distances of roughly $4.5 \mathrm{kpc}$. Sources in this kinematic distance bin correspond to sources within the $5 \mathrm{kpc}$ molecular ring, and we further discuss the drop in fraction with star formation activity in the following section.

The additional gas properties provided by the $\mathrm{NH}_{3}$ survey presented in this work allow for further exploration of physical properties as a function of the star formation activity groups defined by Dunham et al. (2011). We find that the mean $T_{\mathrm{K}}$, $\sigma_{\mathrm{Vlss}}$, and $N_{\mathrm{NH}_{3}}$ increase with star formation activity group such that BGPS sources associated with an EGO or RMS source have higher gas kinetic temperatures, larger observed line widths, and higher $\mathrm{NH}_{3}$ column densities. The mean $T_{\mathrm{K}}$ increases from $13.9 \mathrm{~K}$ in group 0 to $22.7 \mathrm{~K}$ for group 3 , while $\left\langle\sigma_{\mathrm{Vlsr}}\right\rangle$ increases from $0.70 \mathrm{~km} \mathrm{~s}^{-1}$ to $1.10 \mathrm{~km} \mathrm{~s}^{-1}$ from group 0 to 3. Similarly, $\left\langle N_{\mathrm{NH}_{3}}\right\rangle$ increases from $5.6 \times 10^{14} \mathrm{~cm}^{-2}$ for group 0 to $1.6 \times 10^{15} \mathrm{~cm}^{-2}$. See Table 9 for further statistical characterization of each property distribution.

We see a trend of increasing $\left\langle T_{\mathrm{K}}\right\rangle$ with increasing number of associated mid-IR sources such that BGPS sources with a larger number of mid-IR sources are typically warmer than BGPS sources with fewer mid-IR sources (see Figure 23). There is considerable spread in $T_{\mathrm{K}}$ with number of mid-IR sources, and the observed trend is a result of increase in the minimum $T_{\mathrm{K}}$ detected with number of mid-IR sources. The warmest temperatures in our sample are seen in BGPS sources across the entire range of number of mid-IR sources.

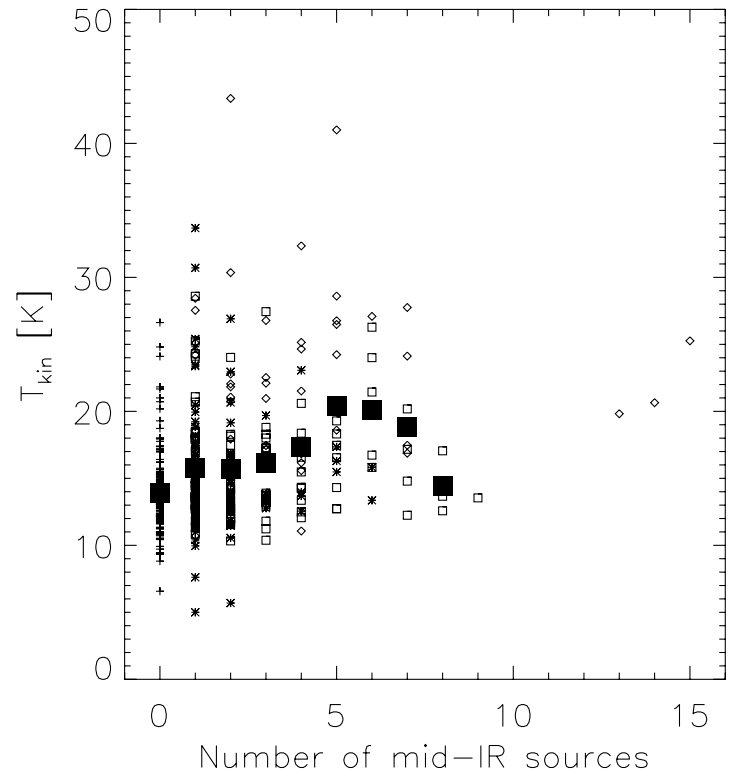

Figure 23. $T_{\mathrm{K}}$ vs. number of mid-IR sources associated with each BGPS source from Dunham et al. (2011). Plus signs are group 0 sources, asterisks are group 1 sources, open squares are group 2, diamonds are group 3, and the large, filled squares mark the mean $T_{\mathrm{K}}$ for each number of mid-IR sources. The $\left\langle T_{\mathrm{K}}\right\rangle$ increases with number of mid-IR sources up to six mid-IR sources, above which we suffer from small number statistics.

We also explore the fraction of BGPS sources with star formation activity as a function of $R_{\mathrm{Gal}}$. The right panel of Figure 22 plots the fraction of BGPS sources with mid-IR signs of star formation versus Galactocentric radius for the 233 sources at the near kinematic distances. The binning is the same as in Figure 19 excluding the sources in Gemini OB1 since the GLIMPSE catalogs do not cover the outer Galaxy. The fraction with mid-IR sources is lowest at the smallest $R_{\mathrm{Gal}}$ and highest at the largest $R_{\mathrm{Gal}}$ and exhibits a $10 \%$ decrease toward the $5 \mathrm{kpc}$ molecular ring.

The decrease in the fraction of BGPS sources associated with a mid-IR source seen in the molecular ring (Figure 22) is not indicative of less star formation activity within the ring. We see a clear peak in sources toward $R_{\mathrm{Gal}}=4-5 \mathrm{kpc}$ (Figure 24) in 


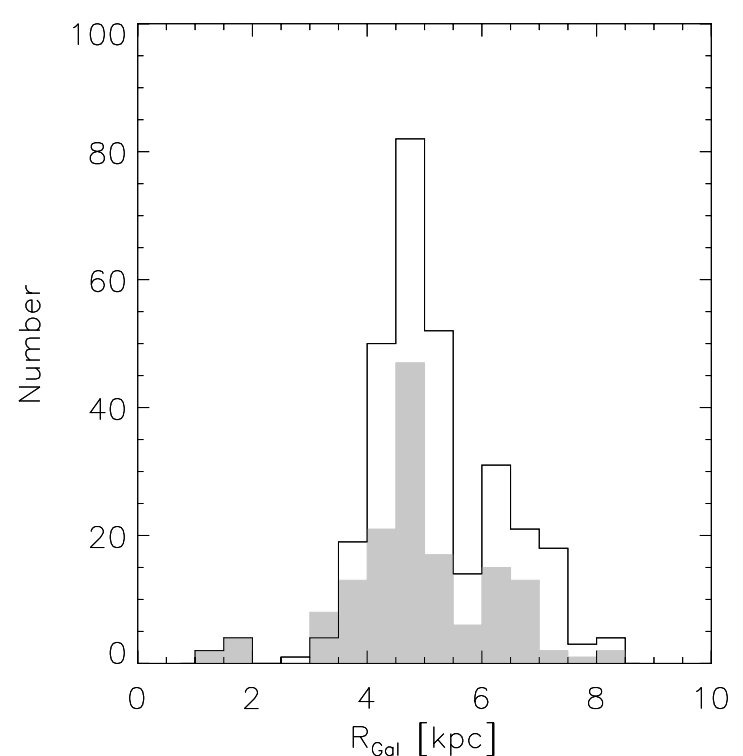

Figure 24. Distributions of BGPS sources with (solid line) and without (shaded gray) star formation activity as described in Dunham et al. (2011).

sources that are associated as well as those that are not associated with a mid-IR source. Although the gas and dust needed to form stars are more abundant in the molecular ring, the fraction of BGPS sources with mid-IR sources is lower. The decrease in the fraction of BGPS sources with mid-IR sources could be a result of a few different effects. The first possibility is the increased mean kinematic distances to the sources within the molecular ring. The limiting luminosity (and mass) of the midIR catalogs considered by Dunham et al. (2011) increases with distance such that only the most luminous (highest mass) YSOs will be detected toward the most distant BGPS sources. The overall increase in the fraction of BGPS sources with a mid-IR source as a function of $R_{\mathrm{Gal}}$ is therefore a result of the decreasing kinematic distance with increasing $R_{\mathrm{Gal}}$ and the rarity of highmass stars and YSOs. Similarly, since the BGPS sources within the molecular ring are located at larger kinematic distances, the lower fraction of mid-IR sources could simply be a result of the higher mass detection limit of the mid-IR catalogs at the larger kinematic distances.

A second possible cause of the lower fraction of sources with mid-IR counterparts is the abundant dense gas within the molecular ring (e.g., Scoville \& Solomon 1975; Burton et al. 1975; Clemens et al. 1988; Kolpak et al. 2002; Rathborne et al. 2009; Roman-Duval et al. 2010), which would provide higher extinction toward the mid-IR sources in this region. Greater extinction would effectively raise the mass detection limit of the YSOs at similar distances. This could reduce the fraction of BGPS sources with a mid-IR counterpart simply because the most massive YSOs are more rare. The third possibility is the increased background of polycyclic aromatic hydrocarbon (PAH) emission toward the longitudes corresponding to the molecular ring. Point sources are easily lost in the increased background $8 \mu \mathrm{m}$ emission and are therefore unlikely to be included in the point-source catalogs. Robitaille et al. (2008) employed a flux cut to avoid this issue and ensure the reliability and completeness of the GLIMPSE red source catalog. However, the mid-IR sources considered by D11 are dominated by an additional GLIMPSE catalog created expressly for that work, which did not include a flux cut and will certainly be sensitive to the background emission. It is likely that the

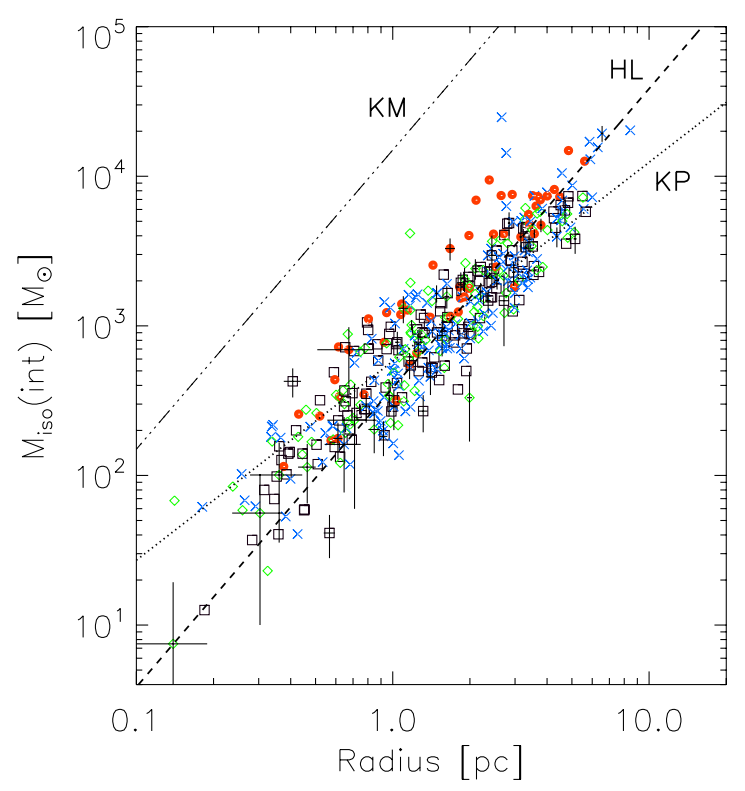

Figure 25. $M_{\text {iso }}$ (int) vs. radius for the full sample of 456 BGPS sources. Symbols and colors denote the star formation activity within each BGPS source as defined by D11. Black squares represent group 0 sources (no corresponding mid-IR sources), blue crosses mark group 1 sources, green diamonds denote group 2 sources, and red circles depict group 3 sources (young RMS sources and EGOs). Error bars are shown for every tenth BGPS source. The dash-dotted line marks the Krumholz \& McKee (2008) surface density threshold, the dotted line marks the Kauffmann \& Pillai (2010) relation, and the dashed line denotes the Heiderman et al. (2010) and Lada et al. (2010) threshold for efficient star formation.

(A color version of this figure is available in the online journal.)

reduced fraction of BGPS sources that coincide with a mid-IR source is the combined result of all three of the effects discussed here.

\subsection{Comparisons to Criteria for Efficient or Massive Star Formation}

In this section, we compare various criteria for efficient or massive star formation with our BGPS sample. We plot mass $\left(M_{\text {iso }}(\right.$ int $)$, Equation (4)) versus radius $(R$, Equation (1)) for the full sample of BGPS sources with distance estimates in Figure 25. Error bars are plotted for every tenth data point to avoid clutter. Points are coded by shape and color according to the four groups discussed in Section 8.3. Note that uncertainties in distance will tend to create a correlation of the form $M \propto r^{2}$.

Heiderman et al. (2010) compared star formation rate surface densities $(\Sigma(S F))$ and gas surface densities $(\Sigma($ gas $))$ for 20 nearby clouds that are forming primarily low-mass stars and a group of more distant dense clumps that are forming massive stars. They found that $\Sigma(S F)$ was a strong nonlinear function of $\Sigma\left(\right.$ gas) up to about $129 \pm 14 M_{\odot} \mathrm{pc}^{-2}$. Above that "threshold," $\Sigma(S F)$ was roughly linear with $\Sigma$ (gas). Independently, Lada et al. (2010) found that the star formation rate in a sample of clouds was linearly proportional to the cloud mass above $\Sigma($ gas $) \sim 116 M_{\odot} \mathrm{pc}^{-2}$, while comparisons to total cloud mass showed no regularity.

In Figure 25, a dotted line of $\Sigma$ (gas) $=122.5 M_{\odot} \mathrm{pc}^{-2}$, or $N_{\mathrm{H}_{2}}^{\text {avg }} \geqslant 6.49 \times 10^{21} \mathrm{~cm}^{-2}$, represents an average of the criteria found by Heiderman et al. (2010) and Lada et al. (2010). We refer to this as the HL criterion for "efficient" star formation. Of the 456 sources in the full sample, 211 (46.3\%) satisfy the HL criterion when we use the surface density of the entire source. 
However, since the BGPS sources have higher surface density structures on smaller scales, BGPS sources that do not satisfy the HL criterion overall may still have subregions that do satisfy it. Thus, $46.3 \%$ should be viewed as a lower limit. If, for example, we consider the peak column density, $N_{\mathrm{H}_{2}}^{\text {beam }}, 86.8 \%$ of the full sample of BGPS sources would have some part that satisfies the HL criterion. Of the BGPS sources with the most certain star formation (Group 3), 70.5\% (36 of 51) have average surface densities above the HL criterion and $98 \%$ (50 of 51) have peak surface densities above the HL criterion.

A dash-dotted line in Figure 25 shows the criterion to avoid excessive fragmentation of $\Sigma$ (gas) $=1 \mathrm{~g} \mathrm{~cm}^{-2}=4787 M_{\odot} \mathrm{pc}^{-2}$ advocated by Krumholz \& McKee (2008) and presented earlier by McKee \& Tan (2003). None of the BGPS sources in our full sample come close to satisfying this KM criterion. As with the HL criterion, parts of these sources may satisfy this criterion. The surveys by Plume et al. (1992) toward regions known to be forming massive stars found quite a few sources satisfying this criterion, though $\mathrm{Wu}$ et al. (2010) found that most exceeded the KM criterion only as measured in the highest density tracers. Since the Plume et al. sources are certainly included in our BGPS survey, they must form the densest parts of the BGPS sources.

Kauffmann et al. (2010) and Kauffmann \& Pillai (2010) have recently suggested a much less stringent criterion for massive star formation than the KM criterion discussed above. They argued that clouds known to be forming massive $\left(M_{*} \sim\right.$ $\left.10 M_{\odot}\right)$ stars have structural properties described by $m(r)>$ $870 M_{\odot}(r / \text { pc })^{1.33}$, where $m(r)$ is the mass within radius $r$ about a peak surface density. Clouds below this criterion are generally not forming massive stars. This "KP" criterion corresponds to a line of different slope in Figure 25 and a criterion for surface density that decreases with radius, $\Sigma($ gas $) \propto r^{-0.67}$. While we have not characterized the internal structure of our BGPS sources, we can compare their total mass and radius to the KP relation. When determining their relationship, KP reduced Ossenkopf \& Henning (1994) dust opacities by a factor of 1.5. Since we have assumed Ossenkopf \& Henning dust opacities without the factor of 1.5 reduction, we scale the mass-radius relation to our assumed dust opacities and use $m(r)>580 M_{\odot}(r / \mathrm{pc})^{1.33}$ for the KP relation appropriate for our mass estimates. In the full sample of BGPS sources, $47.6 \%$ have masses above the KP relation. Among the Group 3 sources, those most certain to be forming stars, and likely to be forming massive stars, $80 \%$ (41 of 51) lie above the KP relation.

Since the BGPS includes sources ranging from cores to clouds, we consider all three star formation criteria discussed above in order to identify all BGPS sources that have high enough densities to be forming stars on vastly different size scales. We estimate that about half the full sample lies above both the HL and KP criteria and should be forming stars, including massive stars, with high efficiency. Smaller parts of a larger fraction $(86.8 \%)$ of BGPS sources may be forming stars efficiently, and a still smaller part may satisfy the KM criterion for massive star formation. As discussed in Section 8.3, we observe mid-IR sources toward $67 \%$ of the full sample. Since the mid-IR catalogs considered by Dunham et al. (2011) include high-mass YSOs across the Galaxy, we are capable of seeing all massive YSO counterparts of BGPS sources. Conversely, the mid-IR catalogs used in Dunham et al. (2011) include only the nearby low-mass YSOs. Therefore, we should not expect to detect mid-IR counterparts toward the full $86.8 \%$ BGPS sources with some surface densities high enough for efficient star formation. Roughly speaking, the statistics of mid-IR source detection are consistent with both the HL and KP relations.

The $47.6 \%$ of BGPS sources that satisfy the KP relation contain $79.3 \%$ of the total mass in the full sample. Thus, the majority of the mass in the BGPS full sample would be predicted by the KP relation to be forming massive stars. These sources are characterized by the following mean properties: $\left\langle\log \left(M_{\text {iso }}\left(120^{\prime \prime}\right) / M_{\odot}\right)\right\rangle=3.29 \pm 0.42,\left\langle\log \left(M_{\text {iso }}(\right.\right.$ int $\left.\left.) / M_{\odot}\right)\right\rangle=$ $3.30 \pm 0.49,\langle R\rangle=2.30 \pm 1.51 \mathrm{pc},\left\langle\log \left(n\left(120^{\prime \prime}\right) / \mathrm{cm}^{-3}\right)\right\rangle=$ $3.29 \pm 0.49,\left\langle\log (n\right.$ (int $\left.\left.) / \mathrm{cm}^{-3}\right)\right\rangle=3.16 \pm 0.56,\langle\Sigma($ int $)\rangle=$ $0.051 \pm 0.036 \mathrm{~cm}^{-2}$, and $\left\langle\log \left(N_{\mathrm{H}_{2}}^{\text {beam }} / \mathrm{cm}^{-2}\right)\right\rangle=22.23 \pm 0.28$, where the standard deviation of the distribution is given as an error representing the spread in each property. The BGPS sources satisfying the KP relation are typically larger, more massive, and less dense than the previous samples of massive star-forming regions chosen based on tracers of star formation that were discussed in Section 1.

\section{SUMMARY}

We present the results of a survey of $\mathrm{NH}_{3}(1,1),(2,2)$, and $(3,3)$ toward a sample of $6311.1 \mathrm{~mm}$ continuum sources from the BGPS. We have detected the $\mathrm{NH}_{3}(1,1)$ line toward 456 BGPS sources $(72 \%)$, demonstrating that the high column density features identified in the BGPS and other continuum studies are excellent predictors of the presence of dense gas. We have determined kinematic distances and resolved the kinematic distance ambiguity (KDA) to all 456 BGPS sources via association with an $8 \mu \mathrm{m}$ IRDC seen in the GLIMPSE mosaics or the presence of HISA in the VGPS or SGPS. We have placed 233 BGPS sources at the near distance, 221 at the far distance, and two at the tangent distance. The kinematic distances range from 0.1 to $15.0 \mathrm{kpc}$.

With the KDA resolved, we calculate physical properties based on both the $1.1 \mathrm{~mm}$ continuum emission (e.g., $R, M_{\text {iso }}$, $n, \Sigma$, and $N_{\mathrm{H}_{2}}$ ) and the $\mathrm{NH}_{3}$ line emission (e.g., $T_{\mathrm{K}}, T_{\mathrm{ex}}, M_{\mathrm{vir}}$, $\left.\sigma_{\mathrm{Vlsr}}, \sigma_{\mathrm{NT}}, N_{\mathrm{NH}_{3}}\right)$. The large range in kinematic distances results in a large range in most physical properties (see Table 8 for a full statistical description of the distribution of each physical quantity). Different scale structures are detected at different distances due to the Malmquist bias and the spatial filtering in the BGPS data, which is a complication that will affect all continuum based studies. Based on the characteristic mass, radius, and density of cores, clumps, and clouds presented by Bergin \& Tafalla (2007), we find that the BGPS predominately detects cores at distances of 1-2 kpc, clumps at distances of 2-7 kpc, and clouds at distances greater than $7 \mathrm{kpc}$. Therefore, the effects of distance must be carefully accounted for.

We have studied the Galactic trends in each physical property as a function of Galactocentric radius, $R_{\mathrm{Gal}}$. We see a strong peak in the number of BGPS sources within the $5 \mathrm{kpc}$ molecular ring, which corresponds to the end of the Galactic bar and beginning of the Scutum spiral arm. We also see a peak in sources at $R_{\mathrm{Gal}} \sim 6 \mathrm{kpc}$, which corresponds to the Sagittarius arm. We have found the following trends in physical properties with $R_{\mathrm{Gal}}$.

1. There are no obvious trends among the inner Galaxy sources. Sources in Gem OB1 have a higher $\left\langle T_{\mathrm{K}}\right\rangle$ than sources within the inner Galaxy, although this could be caused by a bias of the Gem OB1 sources toward later evolutionary stages.

2. $\mathrm{NH}_{3}$ column density decreases with $R_{\mathrm{Gal}}$ with a factor of approximately eight difference between the sources closest to the Galactic center and those in Gem OB1. 
3. Similarly, $\mathrm{NH}_{3}$ abundance also decreases smoothly with $R_{\mathrm{Gal}}$ with a factor of seven difference between the inner and outer most BGPS sources. The $\mathrm{NH}_{3}$ abundance changes with $R_{\mathrm{Gal}}$ at the same rate as the nitrogen abundance, suggesting that the decrease in $\mathrm{NH}_{3}$ abundance is caused by less nitrogen to form $\mathrm{NH}_{3}$ toward the outer Galaxy, although changes in dust properties (e.g., gas-to-dust ratio, grain size, grain temperature) likely also affect the measured $\mathrm{NH}_{3}$ abundance.

4. When comparing sources at similar kinematic distances, there is little variation in physical properties across the Galaxy regardless of environment (inside versus outside the molecular ring, for example). This is in contrast to the molecular clouds seen in the GRS, which were found to be larger, more massive, warmer, and have higher column densities within the molecular ring (Rathborne et al. 2009). This suggests that environment may affect the large-scale molecular clouds while interior regions of dense gas exhibit similar properties regardless of environment.

We have characterized the star formation activity of the BGPS sources based on the presence of an associated mid-IR source as described in Dunham et al. (2011). We find that $67 \%$ of the BGPS sources in this study have an associated mid-IR source. BGPS sources associated with either an EGO (Cyganowski et al. 2008) or an RMS (Hoare et al. 2004; Urquhart et al. 2008) have a higher mean $T_{\mathrm{K}}, \sigma_{\mathrm{Vlsr}}$, and $N_{\mathrm{NH}_{3}}$ than sources without any mid-IR indication of star formation activity.

We also compared the BGPS sources to criteria suggested for both efficient star formation and for massive star formation. We find that $46.3 \%$ have average surface densities above the threshold for efficient star formation (Heiderman et al. 2010; Lada et al. 2010). If we consider the peak column density, we find that $86.8 \%$ of the full sample have some part with surface densities above the threshold for efficient star formation. Similarly, we have compared the BGPS sources to the criterion for massive star formation presented by Kauffmann et al. (2010) and found that $47.6 \%$ of the full sample have masses above their criterion. Thus, roughly half of the full sample satisfies both criteria and should be forming stars with high efficiency, while smaller parts of $86.8 \%$ of the full sample may be forming stars efficiently. This is consistent with the $67 \%$ of the full sample that has corresponding mid-IR sources. The BGPS sources that satisfy the Kauffmann et al. (2010) criterion for massive star formation are, on average, larger, more massive, and less dense than seen in previous large-scale studies of massive star-forming regions that were based on tracers of massive star formation (i.e., Plume et al. 1992, 1997; Shirley et al. 2003; Wu et al. 2010).

The authors thank the anonymous referee for constructive comments, C. Figura and L. Morgan for the $\mathrm{NH}_{3}$ observations they have provided for this work, as well as T. Robitaille and M. M. Dunham for many enlightening conversations. The BGPS is supported by the National Science Foundation through NSF grant AST-0708403. M.K.D. and N.J.E. were supported by NSF grant AST-0607793 to the University of Texas at Austin. M.K.D. was additionally supported by a grant from the National Radio Astronomy Observatory (NRAO) Student Observing Support Program, award number GSSP09-0004. The NRAO is a facility of the National Science Foundation, operated under cooperative agreement by Associated Universities, Inc. E.R. is supported by a Discovery Grant from the Natural Sciences and Engineering Research Council Canada. C.J.C. is supported by an NSF Astronomy and Astrophysics Postdoctoral
Fellowship under award AST-1003134 and also acknowledges support from NSF grant AST-0808119. J.S.U. is supported by a CSIRO OCE postdoctoral grant. This paper made use of information from the Red MSX Source (RMS) survey database at www.ast.leeds.ac.uk/RMS, which was constructed with support from the Science and Technology Facilities Council of the UK. This publication makes use of molecular line data from the Boston University-FCRAO Galactic Ring Survey (GRS). The GRS is a joint project of Boston University and Five College Radio Astronomy Observatory, funded by the National Science Foundation under grants AST-9800334, AST-0098562, and AST-0100793. This research used the facilities of the Canadian Astronomy Data Centre operated by the National Research Council of Canada with the support of the Canadian Space Agency.

\section{REFERENCES}

Aguirre, J. E., Ginsburg, A. G., Dunham, M. K., et al. 2011, ApJS, 192, 4 Anderson, L. D., \& Bania, T. M. 2009, ApJ, 690, 706

Anglada, G., Estalella, R., Pastor, J., Rodriguez, L. F., \& Haschick, A. D. 1996, ApJ, 463, 205

Battersby, C., Bally, J., Gingsburg, A. G., et al. 2011, A\&A, in press (arXiv:1101.4654)

Battersby, C., Bally, J., Jackson, J. M., et al. 2010, ApJ, 721, 222

Benjamin, R. A., Churchwell, E., Babler, B. L., et al. 2003, PASP, 115, 953

Bergin, E. A., \& Tafalla, M. 2007, ARA\&A, 45, 339

Beuther, H., Schilke, P., Menten, K. M., et al. 2002, ApJ, 566, 945

Bohlin, R. C., Savage, B. D., \& Drake, J. F. 1978, ApJ, 224, 132

Burton, W. B., \& Bania, T. M. 1974, A\&A, 33, 425

Burton, W. B., Gordon, M. A., Bania, T. M., \& Lockman, F. J. 1975, ApJ, 202, 30

Burton, W. B., Liszt, H. S., \& Baker, P. L. 1978, ApJ, 219, L67

Busfield, A. L., Purcell, C. R., Hoare, M. G., et al. 2006, MNRAS, 366, 1096

Carey, S. J., Clark, F. O., Egan, M. P., et al. 1998, ApJ, 508, 721

Carey, S. J., Egan, M. P., Kuchar, T. A., et al. 2000, BAAS, 197, 516

Cesaroni, R., Palagi, F., Felli, M., et al. 1988, A\&AS, 76, 445

Chambers, E. T., Jackson, J. M., Rathborne, J. M., \& Simon, R. 2009, ApJS, 181,360

Clemens, D. P., Sanders, D. B., \& Scoville, N. Z. 1988, ApJ, 327, 139

Cohen, R. S., \& Thaddeus, P. 1977, ApJ, 217, L155

Cyganowski, C. J., Whitney, B. A., Holden, E., et al. 2008, AJ, 136, 2391

Dunham, M. K., Robitaille, T. P., Evans, N. J., II, et al. 2011, ApJ, 731, 90

Dunham, M. K., Rosolowsky, E., Evans, N. J., II, et al. 2010, ApJ, 717, 1157

Egan, M. P., Shipman, R. F., Price, S. D., et al. 1998, ApJ, 494, L199

Fish, V. L., Reid, M. J., Wilner, D. J., \& Churchwell, E. 2003, ApJ, 587, 701

Flynn, E. S., Jackson, J. M., Simon, R., et al. 2004, in ASP Conf. Ser. 317, Milky

Way Surveys: The Structure and Evolution of our Galaxy, ed. D. Clemens,

R. Shah, \& T. Brainerd (San Francisco, CA: ASP), 44

Foster, J. B., Rosolowsky, E. W., Kauffmann, J., et al. 2009, ApJ, 696, 298

Friesen, R. K., Di Francesco, J., Shirley, Y. L., \& Myers, P. C. 2009, ApJ, 697, 1457

Gibson, S. J., Taylor, A. R., Higgs, L. A., Brunt, C. M., \& Dewdney, P. E. 2005, ApJ, 626, 195

Gibson, S. J., Taylor, A. R., Higgs, L. A., \& Dewdney, P. E. 2000, ApJ, 540, 851 Goldsmith, P. F., \& Li, D. 2005, ApJ, 622, 938

Gummersbach, C. A., Kaufer, A., Schaefer, D. R., Szeifert, T., \& Wolf, B. 1998, A\&A, 338, 881

Harju, J., Walmsley, C. M., \& Wouterloot, J. G. A. 1993, A\&AS, 98, 51

Heiderman, A., Evans, N. J., II, Allen, L. E., Huard, T., \& Heyer, M. 2010, ApJ, 723, 1019

Hoare, M. G., Lumsden, S. L., Oudmaijer, R. D., et al. 2004, in ASP Conf. Ser. 317, Milky Way Surveys: The Structure and Evolution of our Galaxy, ed. D. Clemens, R. Shah, \& T. Brainerd (San Francisco, CA: ASP), 156

Jackson, J. M., Bania, T. M., Simon, R., et al. 2002, ApJ, 566, L81

Jackson, J. M., Rathborne, J. M., Shah, R. Y., et al. 2006, ApJS, 163, 145

Kennicutt, R. C. 2005, in IAU Symp. 227, Massive Star Birth: A Crossroads of Astrophysics, ed. R. Cesaroni, M. Felli, E. Churchwell, \& M. Walmsley (Cambridge: Cambridge Univ. Press), 3

Kauffmann, J., \& Pillai, T. 2010, ApJ, 723, L7

Kauffmann, J., Pillai, T., Shetty, R., Myers, P. C., \& Goodman, A. A. 2010, ApJ, 716,433

Knapp, G. R. 1974, AJ, 79, 527 
Kolpak, M. A., Jackson, J. M., Bania, T. M., \& Dickey, J. M. 2002, ApJ, 578, 868

Krumholz, M. R., \& McKee, C. F. 2008, Nature, 451, 1082

Lada, C. J., Lombardi, M., \& Alves, J. F. 2010, ApJ, 724, 687

Li, D., \& Goldsmith, P. F. 2003, ApJ, 585, 823

Lumsden, S. L., Hoare, M. G., Oudmaijer, R. D., \& Richards, D. 2002, MNRAS, 336,621

Markwardt, C. B. 2009, in ASP Conf. Ser. 411, Astronomical Data Analysis Software and Systems XVIII, ed. D. A. Bohlender, D. Durand, \& P. Dowler (San Francisco, CA: ASP), 251

Matthews, H., Kirk, H., Johnstone, D., et al. 2009, AJ, 138, 1380

McClure-Griffiths, N. M., Dickey, J. M., Gaensler, B. M., et al. 2005, ApJS, 158,178

McKee, C. F., \& Tan, J. C. 2003, ApJ, 585, 850

Molinari, S., Swinyard, B., Bally, J., et al. 2010, PASP, 122, 314

Motte, F., Bontemps, S., Schilke, P., et al. 2007, A\&A, 476, 1243

Motte, F., Schilke, P., \& Lis, D. C. 2003, ApJ, 582, 277

Mueller, K. E., Shirley, Y. L., Evans, N. J., II, \& Jacobson, H. R. 2002, ApJS, 143,469

Ossenkopf, V., \& Henning, T. 1994, A\&A, 291, 943

Pandian, J. D., Menten, K. M., \& Goldsmith, P. F. 2009, ApJ, 706, 1609

Peretto, N., \& Fuller, G. A. 2009, A\&A, 505, 405

Plume, R., Jaffe, D. T., \& Evans, N. J., II. 1992, ApJS, 78, 505

Plume, R., Jaffe, D. T., Evans, N. J., II, Martin-Pintado, J., \& Gomez-Gonzalez, J. 1997, ApJ, 476, 730

Price, S. D., Egan, M. P., Carey, S. J., Mizuno, D. R., \& Kuchar, T. A. 2001, AJ, 121,2819

Rathborne, J. M., Jackson, J. M., Chambers, E. T., et al. 2005, ApJ, 630, L181

Rathborne, J. M., Jackson, J. M., Chambers, E. T., et al. 2010, ApJ, 715, 310

Rathborne, J. M., Jackson, J. M., \& Simon, R. 2006, ApJ, 641, 389

Rathborne, J. M., Jackson, J. M., Zhang, Q., \& Simon, R. 2008, ApJ, 689, 1141

Rathborne, J. M., Johnson, A. M., Jackson, J. M., Shah, R. Y., \& Simon, R. 2009, ApJS, 182, 131

Rathborne, J. M., Simon, R., \& Jackson, J. M. 2007, ApJ, 662, 1082

Reid, M. J., Menten, K. M., Zheng, X. W., et al. 2009, ApJ, 700, 137
Robinson, B. J., Manchester, R. N., Whiteoak, J. B., et al. 1984, ApJ, 283, L31 Robitaille, T. P., Meade, M. R., Babler, B. L., et al. 2008, AJ, 136, 2413

Rolleston, W. R. J., Smartt, S. J., Dufton, P. L., \& Ryans, R. S. I. 2000, A\&A, 363,537

Roman-Duval, J., Jackson, J. M., Heyer, M., Rathborne, J., \& Símon, R. 2010, ApJ, 723, 492

Roman-Duval, J., Jackson, J. M., Heyer, M., et al. 2009, ApJ, 699, 1153

Rosolowsky, E., Dunham, M. K., Ginsburg, A., et al. 2010, ApJS, 188, 123

Rosolowsky, E. W., Pineda, J. E., Foster, J. B., et al. 2008, ApJS, 175, 509

Schlingman, W. M., Shirley, Y. L., Schenk, D. E., et al. 2011, ApJS, 195, 14

Schuller, F., Menten, K. M., Contreras, Y., et al. 2009, A\&A, 504, 415

Scoville, N. Z., \& Solomon, P. M. 1975, ApJ, 199, L105

Shaver, P. A., McGee, R. X., Newton, L. M., Danks, A. C., \& Pottasch, S. R. 1983, MNRAS, 204, 53

Shirley, Y. L., Evans, N. J., II, Young, K. E., Knez, C., \& Jaffe, D. T. 2003, ApJS, 149,375

Simon, R., Rathborne, J. M., Shah, R. Y., Jackson, J. M., \& Chambers, E. T. 2006, ApJ, 653, 1325

Smartt, S. J., Venn, K. A., Dufton, P. L., et al. 2001, A\&A, 367, 86

Sridharan, T. K., Beuther, H., Schilke, P., Menten, K. M., \& Wyrowski, F. 2002, ApJ, 566, 931

Stil, J. M., Taylor, A. R., Dickey, J. M., et al. 2006, AJ, 132, 1158

Tafalla, M., Santiago-García, J., Myers, P. C., et al. 2006, A\&A, 455, 577

Takano, S., Nakai, N., \& Kawaguchi, K. 2002, PASJ, 54, 195

Urquhart, J. S., Hoare, M. G., Lumsden, S. L., Oudmaijer, R. D., \& Moore, T. J. T. 2008, in ASP Conf. Ser. 387, Massive Star Formation: Observations Confront Theory, ed. H. Beuther, H. Linz, \& T. Henning (San Francisco, CA: ASP), 381

Urquhart, J. S., Hoare, M. G., Purcell, C. R., et al. 2009, A\&A, 501, 539

Urquhart, J. S., Moore, T. J. T., Hoare, M. G., et al. 2011, MNRAS, 410, 1237

Weingartner, J. C., \& Draine, B. T. 2001, ApJ, 548, 296

Wood, D. O. S., \& Churchwell, E. 1989a, ApJS, 69, 831

Wood, D. O. S., \& Churchwell, E. 1989b, ApJ, 340, 265

Wu, J., Evans, N. J., Shirley, Y. L., \& Knez, C. 2010, ApJS, 188, 313

Zinchenko, I., Henning, T., \& Schreyer, K. 1997, A\&AS, 124, 385 